Filipe Careli de Almeida

Planejamento de experimentos para análise da influência do teor de água na incerteza de medição de vazão de óleo com medidores ultrassônicos

Dissertação de Mestrado

Dissertação apresentada como requisito parcial para obtenção do grau de Mestre pelo Programa de PósGraduação em Metrologia (Área de concentração: Metrologia para Qualidade e Inovação) da PUC-Rio.

Orientador: Prof. Carlos Roberto Hall Barbosa Co- orientador : Prof. Elcio Cruz de Oliveira 
Filipe Careli de Almeida

\section{Planejamento de experimentos para análise da influência do teor de água na incerteza de medição de vazão de óleo com medidores ultrassônicos}

Dissertação apresentada como requisito parcial para obtenção do grau de Mestre pelo Programa de PósGraduação em Metrologia (Área de concentração: Metrologia para Qualidade e Inovação) da PUC-Rio. Aprovada pela Comissão Examinadora abaixo assinada.

Prof. Carlos Roberto Hall Barbosa Orientador Programa de Pós-Graduação em Metrologia - PUC-Rio

Prof. Elcio Cruz de Oliveira Co-orientador Programa de Pós-Graduação em Metrologia - PUC-Rio

Prof. Ricardo Queiroz Aucélio Departamento de Química - PUC-Rio

Profa. Verônica Maria de Araujo Calado Universidade Federal do Rio de Janeiro - UFRJ

Prof. Márcio da Silveira Carvalho Coordenador Setorial do Centro Técnico Científico - PUC-Rio 
Todos os direitos reservados. É proibida a reprodução total ou parcial do trabalho sem autorização da universidade, do autor e do orientador.

Filipe Careli de Almeida

Formado em Engenharia de Produção Com Ênfase em Mecânica pela Universidade do Estado do Rio de Janeiro, em 2009. Funcionário da Petrobras, desde 2010, onde atua na área de medição de fluídos e metrologia.

Ficha Catalográfica

Almeida, Filipe Careli de

Planejamento de experimentos para análise da influência do teor de água na incerteza de medição de vazão de óleo com medidores ultrassônicos / Filipe Careli de Almeida; Orientador: Carlos Roberto Hall Barbosa. Coorientador: Elcio Cruz de Oliveira. - 2017.

109 f.: il. ; $30 \mathrm{~cm}$

Dissertação (mestrado) - Pontifícia Universidade Católica do Rio de Janeiro, Programa de Pós-Graduação em Metrologia para a Qualidade e Inovação, 2017.

Incluí bibliografia.

1. Metrologia - Teses. 2. Metrologia. 3. Teor de água no óleo. 4. Fator de calibração. 5. Incerteza. 6. Planejamento de experimentos. I. Barbosa, Carlos Roberto Hall. II. Oliveira, Elcio Cruz de. III. Pontifícia Universidade Católica do Rio de Janeiro. Centro Técnico Científico. Programa de Pós-Graduação em Metrologia para a Qualidade e Inovação. IV. Título. 
Dedico este trabalho à minha família, em especial à minha esposa, e aos amigos por toda compreensão e ajuda durante toda esta jornada de conhecimento. 


\section{Agradecimentos}

À Capes e a PUC-Rio por possibilitar e apoio financeiro necessário para que eu pudesse desenvolver esta pesquisa.

Ao meu orientador, Professor Carlos Roberto Hall Barbosa, por acreditar na minha capacidade e por saber tirar o melhor de seus alunos.

Ao meu co-orientador, Professor Elcio Cruz de Oliveira, por todo o apoio, orientação, disponibilidade e por ter sido um dos grandes responsáveis por eu ter conseguido permanecer neste programa.

À minha esposa, Gabriela, pelo apoio, compreensão, carinho, ajuda e amor durante toda esta jornada.

À minha família, por compreender a ausência em alguns momentos por estar dedicado à pesquisa e por todo o apoio durante esta jornada.

À PUC-RIO e ao PósMQI, especialmente aos professores e funcionários deste, por todo o apoio dado durante o programa deste curso e pelos conhecimentos disponibilizados.

Aos amigos e colegas do PósMQI, do meu trabalho e a todos que me ajudaram de alguma forma, seja com informações ou simplesmente apoio.

Aos aqui citados, os meus sinceros agradecimentos. 


\section{Resumo}

Almeida, Filipe Careli de; Barbosa, Carlos Roberto Hall; Oliveira, Elcio Cruz de. Planejamento de experimentos para análise da influência do teor de água na incerteza de medição de vazão de óleo com medidores ultrassônicos. Rio de Janeiro, 2017. 109p. Dissertação de Mestrado Programa de Pós-Graduação em Metrologia (Área de concentração: Metrologia para Qualidade e Inovação), Pontifícia Universidade Católica do Rio de Janeiro.

O objetivo desta dissertação é avaliar a influência do teor de água e do fator do medidor na incerteza de malhas fiscais de medição de óleo utilizando medidores ultrassônicos. A motivação deste trabalho advém do fato do Regulamento Técnico de Medição de Petróleo e Gás Natural (RTM) existente limitar quais metodologias podem ser utilizadas para realizar a análise de teor de água e qual a incerteza máxima permitida para a malha de medição na qual esta se insere, porém não define a incerteza desta, havendo o risco de se adotar um dos métodos de análise permitidos e, ainda assim, a incerteza de medição da malha ultrapassar a definida pela legislação. Além disso, o RTM adota um critério de repetibilidade para a validação das calibrações dos medidores ultrassônicos, portanto avalia-se também a influência deste na incerteza da malha. A metodologia utilizada foi $o$ planejamento de experimentos, utilizando superfície de resposta. Os resultados demonstram que é possível simular a influência da incerteza da análise da água e do fator do medidor na incerteza da malha. Por fim, as conclusões demonstram que as médias atualmente encontradas para incerteza do teor de água são adequadas para manter a incerteza da malha dentro da permitida pelo RTM, porém com pouca variação pode-se exceder este limite; a influência da incerteza do fator do medidor foi inferior ou similar à do teor de água e, por fim, sugere-se a adoção de um critério limite de incerteza ao invés de um critério de repetibilidade para os medidores ultrassônicos.

\section{Palavras-chave}

Metrologia; Teor de água no óleo; Fator de calibração; Incerteza; Planejamento de Experimentos. 
Abstract

Almeida, Filipe Careli de; Barbosa, Carlos Roberto Hall (Advisor); Oliveira, Elcio Cruz de (Co-Advisor). Design of experiments to analyze the influence of water on the uncertainty of oil flow measurement with ultrasonic meters. Rio de Janeiro, 2017. 109p. Dissertação de Mestrado - Programa de Pós-Graduação em Metrologia (Área de concentração: Metrologia para Qualidade e Inovação), Pontifícia Universidade Católica do Rio de Janeiro.

The aim of this dissertation is to evaluate the influence of water content dissolved in crude oil and the meter factor on the uncertainty of the fiscal oil metering using ultrasonic flow meters. The development of this work is motivated by the fact that the Technical Regulation for the Measurement of Oil and Natural Gas (RTM) limits which methodologies can be used for water analysis and what is the maximum permissible uncertainty for the oil flow meter in which it is inserted. However, the RTM doesn't define the uncertainty of the water analysis. There is a risk of adopting one of the permitted methods of analysis and, even so, the measurement uncertainty of the flow meter exceeds the defined by the legislation. In addition, the RTM adopts a repeatability standard when validating the calibration of ultrasonic meters, thus the influence of this on oil uncertainty is also evaluated. The methodology used is the design of experiments and response surface. The results demonstrate that the influence of the water content uncertainty analysis and of the meter factor on the uncertainty of the oil flow meter can be simulated. Finally, the conclusions show that the average currently found for uncertainty of water is adequate to maintain the oil uncertainty within the allowed by the RTM, but small variations may cause this limit to be exceeded. The influence of the meter factor uncertainty was inferior or similar to the water content and, finally, it is suggested the adoption of a standard limit of uncertainty instead of a standard of repeatability for the ultrasonic meters.

\section{Keywords}

Metrology; Water in crude oils; Meter Factor; Uncertainty; Design of Experiments. 


\section{Sumário}

1 Introdução 15

1.1 Contexto 15

1.2 Objetivos Geral e Específicos 16

$\begin{array}{ll}1.3 \text { Motivação } & 17\end{array}$

$\begin{array}{ll}1.4 \text { Estrutura da Dissertação } & 17\end{array}$

2 Medição do teor de água em óleo 19

2.1 Medição de teor de água e a regulamentação brasileira (RTM) 19

$\begin{array}{ll}2.2 \text { Método de Centrifugação } & 21\end{array}$

$\begin{array}{ll}2.3 \text { Método Karl Fischer } & 27\end{array}$

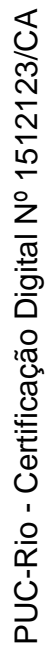

2.4 Analisadores Automáticos de Teor de Água 32

3 Planejamento de Experimentos 34

3.1 Definição 34

3.2 Planejamento Fatorial $\quad 35$

3.3 Superfície de Resposta 36

3.4 Aplicação em um modelo quadrático 38

3.5 Planejamento Composto Central 40

4 Descrição e Incerteza da Malha 43

4.1 Medição de Vazão de óleo pelo Princípio Ultrassônico 43

4.2 Sistema de Medição 45

4.3 Incerteza da Malha Fiscal $\quad 47$

4.4 Incerteza da Análise de Teor de Água 49

4.4.1 Incerteza do Método de Centrifugação 50

4.4.2 Incerteza do Método Karl Fischer 55

5 Desenvolvimento dos experimentos $\quad 60$

5.1 Montagem dos experimentos 60

5.2 Resultados dos experimentos e superfície de resposta 64 
5.3 Análise dos experimentos

6 Conclusões e recomendações 85

6.1 Conclusões 85

6.2 Recomendações para trabalhos futuros 86

7 Referências bibliográficas $\quad 87$

APÊNDICE A - Teste de Normalidade das Amostras 91 


\section{Lista de figuras}

Figura 1 - Figura adaptada de ABNT NBR 14647:2010.

Figura 2 - Curva de precisão para método de teor de água em petróleo segundo ABNT 14647:2010

Figura 3 - Tubo de 167 mm. Figura adaptada do Guia API MPMS 10.4.

Figura 4 - Exemplo de superfície de resposta teórica aplicada a um processo químico.

Figura 5 - Exemplo de Planejamento Composto Central com três variáveis em dois níveis (Lemos et al., 2012).

Figura 7 - Exemplo de malha de vazão utilizando o princípio ultrassônico (Salgado, 2009)

Figura 9 - Gráfico de Pareto das estimativas de efeito padronizado - Malha 1.

Figura 10 - Gráfico de Resíduos x Valores Previstos para Malha 1.

Figura 11 - Gráfico Valores Observados x Valores Previstos para Malha 1.

Figura 12 - Superfície de Resposta para a Malha 1.

Figura 13 - Curva de Nível - Malha 1.

Figura 14 - Gráfico de Pareto das estimativas de efeito padronizado - Malha 2.

Figura 15 - Gráfico de Resíduos x Valores Previstos para Malha 2.

Figura 16 - Superfície de Resposta para a Malha 2.

Figura 17 - Curva de Nível - Malha 2.

Figura 18 - Gráfico de Pareto das estimativas de efeito padronizado - Malha 3.

Figura 19 - Gráfico de Resíduos x Valores Previstos para Malha 3.

Figura 20 - Superfície de Resposta para a Malha 3.

Figura 21 - Curva de Nível - Malha 3. 
Figura 22 - Gráfico de Pareto das estimativas de efeito padronizado - Malha 4.

Figura 23 - Gráfico de Resíduos x Valores Previstos para Malha 4.

Figura 24 - Superfície de Resposta para a Malha $4 . \quad 75$

Figura 25 - Curva de Nível - Malha $4 . \quad 75$

Figura 26 - Gráfico de Pareto das estimativas de efeito padronizado - Malha 5.

Figura 27 - Gráfico de Resíduos x Valores Previstos para Malha 5.

Figura 28 - Superfície de Resposta para a Malha $5 . \quad 78$

Figura 29 - Curva de Nível - Malha $5 . \quad 78$

Figura 30 - Gráfico de Pareto das estimativas de efeito padronizado - Malha 6.

Figura 31 - Gráfico de Resíduos x Valores Previstos para Malha 6.

Figura 32 - Superfície de Resposta para a Malha 6.

Figura 33 - Curva de Nível - Malha 6. 


\section{Lista de tabelas}

Tabela 1 - Tolerância de calibração do tubo cônico, conforme ABNT NBR 14647:2010

Tabela 3 - Expressão de resultados para tubos de $100 \mathrm{ml}$ e de 200 partes, conforme guia API MPMS 10.4

Tabela 4 - Precisão em cada intervalo, conforme Guia API MPMS 10.7. 30

Tabela 5 - Precisão em cada intervalo, conforme Norma API MPMS 10.9. 32

Tabela 6 - Matriz de Planejamento Composto Central com três variáveis. $\quad 41$

Tabela 7 - Influência de cada fator na incerteza das malhas estudadas, de 1 a 6.

Tabela 8 - Variação dos valores dos componentes da incerteza da Malha 1 em $50 \%$, em m3.

Tabela 9 - Experimento incerteza de água x incerteza da variação do MF para a Malha 1.

Tabela 11 - Tabela ANOVA calculada para os termos da regressão da Malha 1.

Tabela 12 - Resultados do Experimento para Malha 1 com variação de $20 \%$.

Tabela 13 - Resultados do Experimento para Malha 1 com variação de $35 \%$.

Tabela 14 - Valores máximos e mínimos do experimento, para Malha 1.

Tabela 15 - Tabela ANOVA calculada para os termos da regressão da Malha

2.

Tabela 16 - Resultados do Experimento para Malha 2

Tabela 17 - Tabela ANOVA calculada para os termos da regressão da Malha 3.

Tabela 18 - Resultados do Experimento para Malha 3.

Tabela 19 - Tabela ANOVA calculada para os termos da regressão da Malha 4. 
Tabela 20 - Resultados do experimento para Malha 4.

Tabela 21 - Tabela ANOVA calculada para os termos da regressão da Malha 5.

Tabela 22 - Resultados do Experimento para Malha 5.

Tabela 23 - Resultados do Experimento para Malha 5 com variação de 35 \%.

Tabela 24 - Tabela ANOVA calculada para os termos da regressão da Malha 6.

Tabela 25 - Resultados do Experimento para Malha 6.

Tabela 26 - Resultados do Experimento para Malha 6 com variação de $35 \%$. 


\section{Lista de Siglas}

\section{Símbolo}

ABNT

ANP

API

ASTM

BSW

CDD

CONMETRO

Fcr

GUM

INMETRO

INPM

ISSO

MF

MPMS

NBR

PUC-Rio

RPM

RSM

RTM

SI

SINMETRO

\section{Descrição}

Associação Brasileira de Normas Técnicas

Agência Nacional do Petróleo, Gás Natural e Biocombustíveis

American Petroleum Institute

American Society for Testing and Materials

Basic Sediments and Water

Central Composite Desing

Conselho Nacional de Metrologia, Normalização e Qualidade

Industrial

Força centrífuga relativa

Guide to the expression of uncertainty in measurement

Instituto Nacional de Metrologia, Qualidade e Tecnologia

Instituto Nacional de Pesos e Medidas

International Organization for Standardization

Meter Factor

Manual of Petroleum Measurement Standards

Denominação de norma da Associação Brasileira de Normas

Técnicas

Pontifícia Universidade Católica do Rio de Janeiro

Rotações por minuto

Response Surface Methodology

Regulamento Técnico de Medição de Petróleo e Gás Natural

Sistema Internacional de Unidades

Sistema Nacional de Metrologia, Normalização e Qualidade

Industrial 


\section{Introdução}

\section{1}

\section{Contexto}

Em se tratando da produção de petróleo em plataformas marítimas, a presença de água e gás associados ao óleo produzido é comum (Thomas, 2001). A fím de separar estas três fases utiliza-se uma série de processos, baseados principalmente em separação gravitacional, pois o gás natural tem valor comercial diferenciado do óleo e a água produzida não tem interesse econômico, podendo causar diversos problemas, como incrustações nas tubulações por depósito de sais e corrosão. Ao fim destas etapas, porém, a separação destas três fases não é completa, e o óleo exportado da plataforma para navios ou oleodutos ainda possui resíduos de água.

No Brasil, a produção de petróleo é regida pelo Regulamento Técnico de Medição de Petróleo e Gás Natural (Resolução Conjunta ANP/INMETRO n. ${ }^{\circ}$ 001, 2013). Este RTM define BSW, Basic Sediments and Water, como sendo a porcentagem de água e sedimentos em relação ao volume total do fluido medido. Neste trabalho, serão analisados casos relacionados à medição fiscal utilizando medidores ultrassônicos em plataformas offshore de produção de petróleo, cujo BSW é composto especificamente por resíduos de água.

O regulamento supracitado limita quais metodologias podem ser utilizadas para realizar a análise de teor de água em óleo e qual a incerteza máxima permitida para a malha de medição na qual este processo se insere. Entretanto, o RTM não indica as incertezas permitidas para os métodos utilizados para esta análise.

Neste cenário, há o risco de se adotar um dos métodos de análise permitidos pelo regulamento e, ainda assim, a incerteza de medição da malha ultrapassar a definida pela legislação. Contudo, a partir da análise da influência (sensibilidade) do teor de água em óleo na incerteza de medição da malha, é possível garantir o atendimento à legislação. Finalmente, a partir das incertezas de medição é possível avaliar metrologicamente a compatibilidade entre os métodos e comparar aspectos 
econômicos e operacionais, que são parâmetros úteis à escolha do método mais adequado a cada situação.

A metodologia de planejamento de experimentos permite observar quais variáveis exercem maior influência nas respostas de um sistema e se há interação entre as variáveis estudadas (Neto et al., 1996). Portanto, o planejamento de experimentos foi escolhido como ferramenta nesta dissertação para avaliar uma possível minimização da incerteza de medição do teor de água em óleo e analisar o critério-limite de aceitação da calibração de medidores de vazão, neste caso especificamente do tipo ultrassônico.

Neste trabalho será estudada a influência de cada grandeza de entrada no cálculo de incerteza na medição de vazão de óleo por medidores do tipo ultrassônico. Em seguida, os fatores mais relevantes serão otimizados por planejamento de experimentos. Uma avaliação preliminar, pautada somente na experiência, sugere que os fatores teor de água no óleo e critério-limite de aceitação da calibração dos medidores de vazão possam ser os mais críticos, o que será discutido neste trabalho.

Esta dissertação utiliza especificamente o planejamento fatorial, que permite avaliar simultaneamente o efeito de várias variáveis a partir de um número reduzido de ensaios experimentais quando comparado a outros métodos (Cunico et al., 2008).

\section{2}

\section{Objetivos Geral e Específicos}

O objetivo geral desta dissertação é definir quais os métodos mais adequados para determinação do teor de água, na perspectiva de otimizar a análise das incertezas de medição da malha de óleo, em atendimento à regulamentação brasileira.

Para tal, foram definidos como objetivos específicos:

- Identificar nas normas existentes como devem ser calculadas as incertezas de medição dos métodos de análise de teor de água aprovados pela legislação e seu impacto na incerteza da malha fiscal de petróleo na qual esta se aplica;

- $\quad$ Analisar, por meio de planejamento de experimentos, a influência do fator de calibração dos medidores do tipo ultrassônico na incerteza da 
malha, uma vez otimizada a análise das incertezas de medição de teor de água para atender à regulamentação existente; e

- Identificar, para medição fiscal, quais métodos de análise de teor de água existentes atendem à incerteza de medição definida no RTM.

\section{3 \\ Motivação}

Os resultados desta dissertação poderão trazer os seguintes benefícios para as partes interessadas:

- Definir quais métodos aprovados pelo RTM atendem à regulação existente, permitindo assim a aplicação do método mais econômico;

- Evitar não conformidades junto à Agência Nacional do Petróleo, Gás Natural e Biocombustíveis (ANP) devido à utilização de métodos aprovados, porém que gerem incertezas de medição da malha maiores que as permitidas;

- Permitir efetuar a análise de dados reais dos cálculos de incerteza de medição de pontos de medição de óleo físcal;

- Permitir avaliar o critério-limite de aceitação da calibração de medidores do tipo ultrassônico, segundo fator que mais afeta a incerteza; e

- Gerar subsídios para aprovar outros métodos e equipamentos de análise de teor de água em óleo, uma oportunidade de melhoria ao RTM.

\section{4}

\section{Estrutura da Dissertação}

A presente dissertação foi estruturada em seis capítulos, sendo este o primeiro capítulo introdutório, e os demais conforme descrito a seguir:

Capítulo 2 - Descreve a importância do controle do teor de água para a legislação e os métodos aprovados por esta para a medição do teor de água em petróleos.

Capítulo 3 - Define as ferramentas utilizadas para otimização da incerteza neste trabalho: planejamento de experimentos, planejamento fatorial, planejamento composto central e superfície de resposta. 
Capítulo 4 - Descreve como devem ser calculadas a incerteza de medição da malha de óleo e a incerteza de medição dos métodos aprovados e estuda e identifica os fatores de maior relevância no cálculo da incerteza da malha de óleo fiscal.

Capítulo 5 - Descreve a coleta, tratamento e análise dos dados visando à otimização da incerteza de medição e avaliação do critério-limite de aceitação.

Capítulo 6 - Apresenta as conclusões e recomendações finais do presente trabalho. 


\section{2 \\ Medição do teor de água em óleo}

\section{1 \\ Medição de teor de água e a regulamentação brasileira (RTM)}

A Agência Nacional de Petróleo, Gás Natural e Biocombustíveis (ANP), instituída em 1988, é o órgão oficial de regulação da indústria do petróleo e derivados no Brasil. Esta é uma autarquia federal vinculada diretamente ao Ministério de Minas e Energia e é regida pela Lei do Petróleo (Lei no 9.478/1977).

A ANP (2016) é a Agência responsável por regular o funcionamento e comércio de petróleo e seus derivados, promover as licitações e contratos ligados à exploração e comercialização destes em nome da União e fiscalizar as atividades desta indústria.

O Instituto Nacional de Metrologia, Qualidade e Tecnologia (Inmetro), também é uma autarquia federal, instituída em 1973 (para substituir o INPM Instituto Nacional de Pesos e Medidas), porém este é vinculado ao Ministério do Desenvolvimento, Indústria e Comércio Exterior. Este órgão atua como Secretaria Executiva do Conselho Nacional de Metrologia, Normalização e Qualidade Industrial (Conmetro), que é o órgão normativo do Sistema Nacional de Metrologia, Normalização e Qualidade Industrial (Sinmetro).

Segundo o Inmetro (2016), a este é atribuído executar as políticas nacionais de Metrologia, verificar as observâncias das normas técnicas e legais, manter os padrões nacionais das unidades de medida e a cadeia de rastreabilidade dos padrões, promover a participação do país nas atividades e conselhos internacionais de Metrologia e planejar e executar a atividade de acreditação dos laboratórios de calibração e ensaio no Brasil.

Estes dois órgãos emitiram a Resolução Conjunta ANP/Inmetro de $n .^{0} 1$, de 10 de Junho de 2013, a qual contém a versão vigente durante o desenvolvimento desta dissertação do Regulamento Técnico de Medição de Petróleo e Gás Natural (RTM).

Este regulamento, em seu item 7.1.2, define que: 
"Os pontos de medição fiscal a serem submetidos para aprovação da ANP devem estar localizados imediatamente após as instalações de separação utilizadas para especificar o $B S W$, estabilizar o petróleo e garantir a remoção de líquidos na corrente de gás natural..."

Em seu item 7.1.7.1, define que a medição fiscal, salvo exceções autorizadas, deverá operar com BSW abaixo de $1 \%$ :

"Nos casos em que a medição de petróleo for realizada com BSW superior a $1 \%$, o agente regulado deverá solicitar a aprovação da ANP, devendo ser justificado o motivo."

Em seu Anexo B - Periodicidade de Calibração, Inspeção e Análise, define que a análise de BSW para pontos fiscais deve ser diária, quando realizada em linha (caso no qual se enquadra este estudo), ou a cada medição, quando esta é realizada em tanque.

No item 8.1.3, define que a determinação da fração volumétrica de água e sedimentos deve seguir o que é especificado nos seguintes documentos:

- ABNT-14236/2002: Produtos de Petróleo e Materiais Betuminosos Determinação do Teor de Água por Destilação;

- $\quad$ ABNT-NBR 14647/2001: Produtos de Petróleo - Determinação da Água e Sedimentos em Petróleos e Óleos Combustíveis pelo Método de Centrifugação;

- $\quad$ ASTM D4007/2008: Standard Test Method for Water and Sediment in Crude Oil by the Centrifuge Method (Laboratory Procedure);

- $\quad$ API/MPMS 10.1/2002: Standard Test Method for Sediment in Crude Oils and Fuel Oils by the Extraction Method, Second Edition;

- $\quad$ API/MPMS 10.4/1999: Determination of Water and Sediment in Crude Oil by Centrifuge Method (Field Procedure); e

- $\quad$ API/MPMS 10.7/2002: Standard Test Method for Water in Crude Oil by Karl Fischer. Titration (Potentiometric).

Por fim, em seu item 8.1.3.1, o regulamento autoriza a utilização de analisadores em linha para a medição contínua do teor de água, desde que os mesmos sejam calibrados periodicamente utilizando os resultados das análises laboratoriais.

Analisando as normas listadas, conclui-se que, para o óleo fiscal produzido, é permitida a utilização dos seguintes métodos para análise de teor de água: Método 
de extração por tolueno, centrifugação e Karl Fisher volumétrico. A norma ASTM 14236, cujo conteúdo trata de determinação do teor de água por destilação, é específica para materiais betuminosos (asfaltos, alcatrões, óleos graxos), não sendo aplicável neste caso.

Em nenhuma das plataformas avaliadas neste estudo é utilizado o método de extração por tolueno, pois este é adequado somente para até 0,4 \% de teor de água. Este deve seguir a norma ASTM 10.1, cuja versão citada no RTM é de 2002. No entanto, a mesma possui atualização no ano de 2007, revisada em 2012.

\section{2 \\ Método de Centrifugação}

A norma ABNT 14647 é citada no RTM em sua versão de 2001, porém a mesma passou por atualização no ano de 2010. Esta norma tem como escopo o ensaio laboratorial para determinação de teor de água utilizando o método de centrifugação, que consiste em colocar porções iguais de óleo e tolueno saturado em água (ou outro solvente, dependendo do tipo de óleo testado) em tubos específicos e, após a centrifugação, medir o volume de água e sedimentos no fundo do mesmo. Quando necessário, pode-se adicionar desemulsificante para facilitar a separação e facilitar a visualização da interface óleo-água.

A fim de manter a temperatura constante de $(60 \pm 3){ }^{\circ} \mathrm{C}$, durante todo o processo de medição, a centrífuga utiliza um banho (bloco metálico ou banho líquido). O ensaio deve utilizar um tubo cônico, de 203 mm, conforme Figura 1, devendo respeitar as tolerâncias de erro de escala estabelecidas na Tabela 1.

$\mathrm{O}$ equipamento deve fornecer uma força centrífuga relativa (fcr) entre $500 \mathrm{e}$ 800 na extremidade dos tubos. É possível calcular a fcr utilizando a velocidade de rotação da mesma por meio de

$$
r p m=1335 \sqrt{f c r / d}
$$

sendo:

$r p m$ - rotações por minuto $\left(\mathrm{rpm}^{1}\right)$;

$f c r$ - força centrífuga relativa em milimetros rotações por minuto ao quadrado $\left(\mathrm{mm} \mathrm{rpm}^{2}\right) ; \mathrm{e}$

\footnotetext{
${ }^{1}$ Embora a norma utilize RPM, esta unidade não está de acordo com o Sistema Internacional de Unidades (SI). A conversão para as unidades do SI é $1 \mathrm{RPM}=2 \pi / 60 \mathrm{rad} . \mathrm{s}^{-1}$.
} 
$d$ - distância em milimetros $(\mathrm{mm})$ entre as extremidades dos dois tubos quando em rotação.

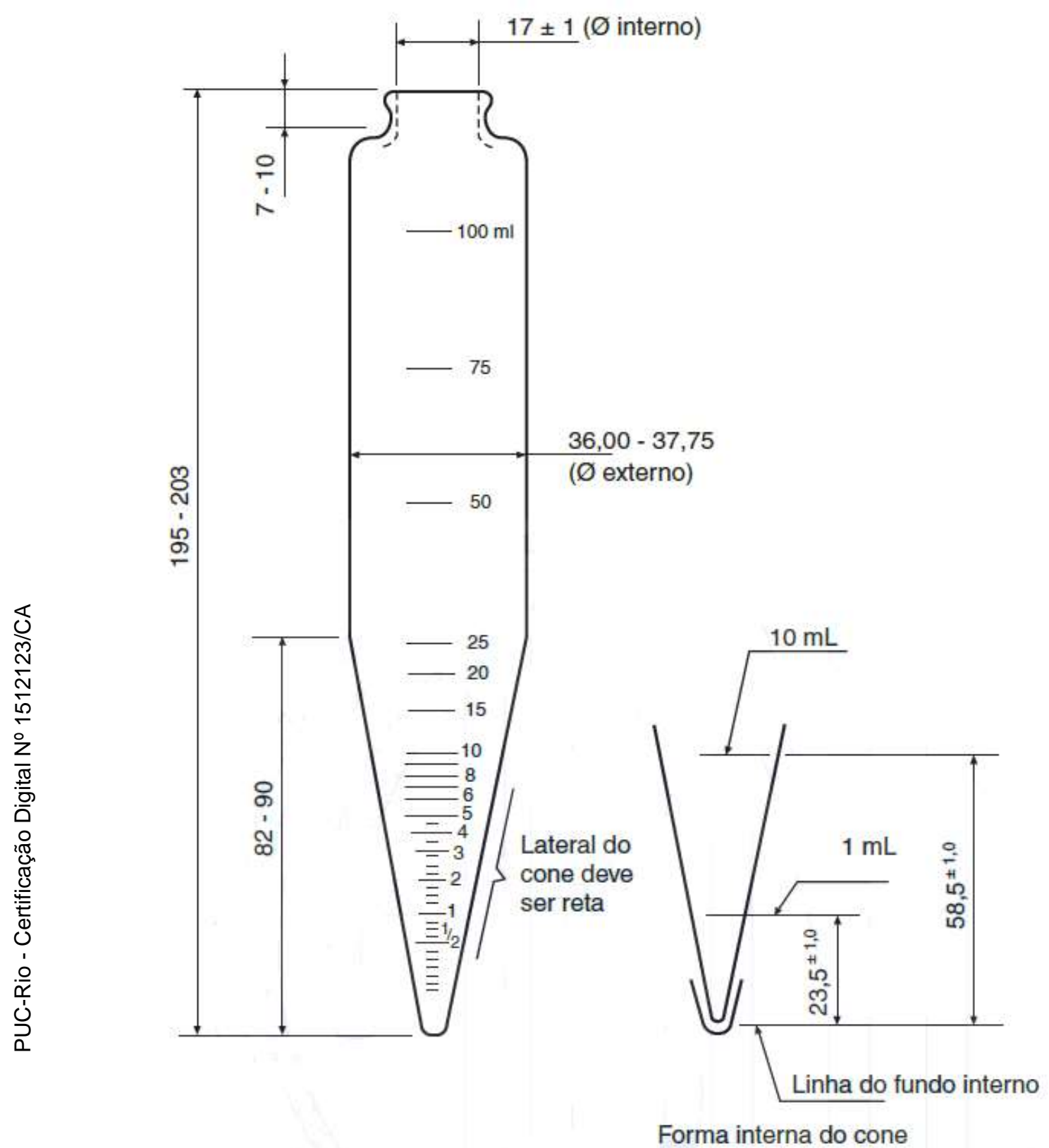

Figura 1 - Figura adaptada de ABNT NBR 14647:2010.

A amostragem deve seguir as normas ABNT NBR 14883 e ASTM D 5854.

São feitas tantas centrifugações quanto necessárias até que o volume de água seja constante após duas medições consecutivas. A aproximação das medições abaixo de $0,1 \mathrm{ml}$ é de 0,025 $\mathrm{ml}$. Entre $0,1 \mathrm{ml}$ e $1 \mathrm{ml}$ é de $0,05 \mathrm{ml}$. Acima de $1 \mathrm{ml}$ é de $0,1 \mathrm{ml}$. Se a diferença entre as quantidades de água e sedimentos dos dois tubos for inferior a $0,025 \mathrm{ml}$, para medições até $0,10 \mathrm{ml}$, calcula-se o teor de água. 
Tabela 1 - Tolerância de calibração do tubo cônico, conforme ABNT NBR 14647:2010

\begin{tabular}{|c|c|c|}
\hline $\begin{array}{c}\text { Faixa } \\
(\mathbf{m l})\end{array}$ & $\begin{array}{c}\text { Subdivisão } \\
(\mathbf{m l})\end{array}$ & $\begin{array}{c}\text { Tolerância de Volume } \\
(\mathbf{m l})\end{array}$ \\
\hline $\mathbf{0}$ a $\mathbf{0 , 1}$ & 0,05 & $\pm 0,02$ \\
\hline Acima de $\mathbf{0 , 1}$ a $\mathbf{0 , 3}$ & 0,05 & $\pm 0,03$ \\
\hline Acima de $\mathbf{0 , 3}$ a $\mathbf{0 , 5}$ & 0,05 & $\pm 0,05$ \\
\hline Acima de $\mathbf{0 , 5}$ a $\mathbf{1 , 0}$ & 0,10 & $\pm 0,10$ \\
\hline Acima de $\mathbf{1 , 0}$ a $\mathbf{2 , 0}$ & 0,10 & $\pm 0,10$ \\
\hline Acima de $\mathbf{2 , 0}$ a 3,0 & 0,20 & $\pm 0,20$ \\
\hline Acima de $\mathbf{3 , 0}$ a 5,0 & 0,50 & $\pm 0,50$ \\
\hline Acima de 5,0 a 10 & 1,00 & $\pm 1,00$ \\
\hline Acima de $\mathbf{1 0}$ a $\mathbf{2 5}$ & 5,00 & $\pm 1,00$ \\
\hline Acima de $\mathbf{2 5}$ a $\mathbf{1 0 0}$ & 25,00 & \\
\hline
\end{tabular}

Para o cálculo da porcentagem em volume de água da amostra utiliza-se

$$
V=\left[\frac{V_{s 1}+V_{s 2}}{V_{t 1}+V_{t 2}}\right] * 100
$$

sendo:

$V$ - porcentagem em volume por volume de água da amostra;

$V_{s l}$ - volume de água e sedimentos no tubo 1, em mililitros (ml);

$V_{s 2}$ - volume de água e sedimentos no tubo 2, em mililitros (ml);

$V_{t 1}$ - volume da amostra no tubo 1, em mililitros (ml);

$V_{t 2}$ - volume da amostra no tubo 2, em mililitros (ml);

Os valores abaixo de $0,05 \%$ devem ser anotados como $0 \%$, quando for mais próximo a este, ou 0,05\%. Acima de 2,00\%, aproxima-se em 0,10\%. Entre 0,20 $\%$ e 2,00\%, aproxima-se em 0,05\%. Abaixo de 0,20\%, utiliza-se a Tabela 2.

Segundo esta norma, para uma probabilidade de $95 \%$, devem ser utilizados os seguintes critérios para julgar a aceitabilidade: a repetibilidade $(r)$, que é a diferença entre os resultados de ensaios sucessivos feitos pelo mesmo operador, com a mesma aparelhagem e com as mesmas condições em amostra de material idêntico, não podendo exceder os valores da Figura 2 para petróleo (na norma ainda é utilizado o termo descontinuado repetitividade); a reprodutibilidade (R), que é a diferença entre dois ensaios individuais e independentes, obtidos por operadores diferentes trabalhando em laboratórios distintos e em amostras de material idênticas, não podendo exceder os valores da Figura 2 para petróleo. 
Tabela 2 - Resultado de água e sedimentos, segundo ABNT NBR 14647:2010

\begin{tabular}{|c|c|c|}
\hline \multicolumn{2}{|c|}{ Volume de água e sedimentos $(\mathbf{m l})$} & \multirow{2}{*}{$\begin{array}{c}\text { Total de água e } \\
\text { sedimentos, \% V/V }\end{array}$} \\
\cline { 1 - 2 } Tubo 1 & Tubo 2 & 0 \\
\hline Não visível & Traços & 0,025 \\
\hline Não visível & 0,025 & 0,05 \\
\hline $\mathbf{0 , 0 2 5}$ & 0,025 & 0,075 \\
\hline $\mathbf{0 , 0 2 5}$ & 0,05 & 0,10 \\
\hline $\mathbf{0 , 0 5}$ & 0,05 & 0,125 \\
\hline $\mathbf{0 , 0 5}$ & 0,075 & 0,15 \\
\hline $\mathbf{0 , 0 7 5}$ & 0,075 & 0,175 \\
\hline $\mathbf{0 , 0 7 5}$ & 0,10 & \\
\hline
\end{tabular}

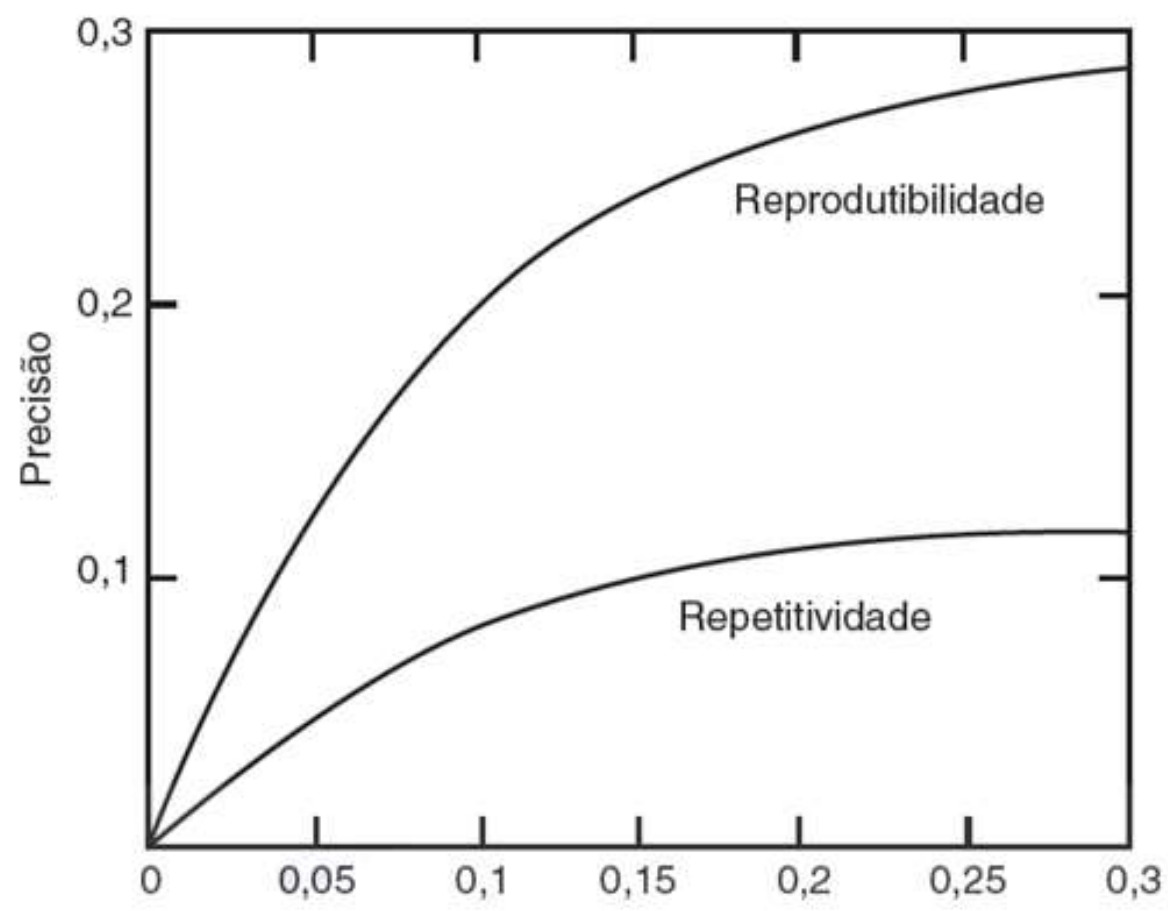

Porcentagem de água e sedimentos

Figura 2 - Curva de precisão para método de teor de água em petróleo segundo ABNT 14647:2010

A norma ABNT NBR 14647 é baseada nas normas ASTM D 1796:2009 e ASTM D 4007:2008, sendo esta segunda citada no RTM como documento a ser seguido.

A ASTM D 4007:2008 apresenta pouca diferença em relação ao método descrito na NBR 14647/2010. Destacam-se os principais pontos divergentes citados na segunda norma: 
- A recomendação de não utilizar este método para óleos que contenham álcoois solúveis em água;

- A indicação de que a força centrífuga relativa deve ter um valor mínimo de $600 \mathrm{~mm} \mathrm{rpm}^{2}$;

- $\quad$ Cita que a amostragem deve seguir as normas ASTM D 4057 e D 4177;

- $\quad$ A inclusão da temperatura de $(71 \pm 3){ }^{\circ} \mathrm{C}$ para o banho de alguns tipos de óleos crus específicos; e

- $\quad$ Cita que, para valores de teor de água entre $0,3 \%$ a $1,0 \%$, a repetibilidade deve ser de $0,12 \%$ e a reprodutibilidade de $0,28 \%$.

O RTM, por último, recomenda seguir a API/MPMS 10.4/1999. Durante a execução deste estudo utilizou-se a versão de 2013, com as correções de 2015. Este guia descreve o método para teste em campo e afirma que os seus resultados nem sempre são os mais exatos. É recomendável, para casos em que sejam necessários resultados com maior exatidão, que se utilize o guia API MPMS 10.3 (ASTM D 4007), voltado para laboratórios.

Este guia utiliza uma mistura de desemulsificante e solvente junto ao óleo na centrífuga a uma temperatura de $(60 \pm 3){ }^{\circ} \mathrm{C}$; entretanto, para alguns óleos crus deve-se utilizar a temperatura de $(71 \pm 3){ }^{\circ} \mathrm{C}$. Os solventes recomendados são querosene, solvente de Stoddard (uma mistura de alcanos e hidrocarbonetos aromáticos), tolueno e xileno. A escolha do tipo de solvente dependerá das características do óleo a ser analisado. Este guia também especifica um tubo de 167 mm, conforme ilustrado na Figura 3. 


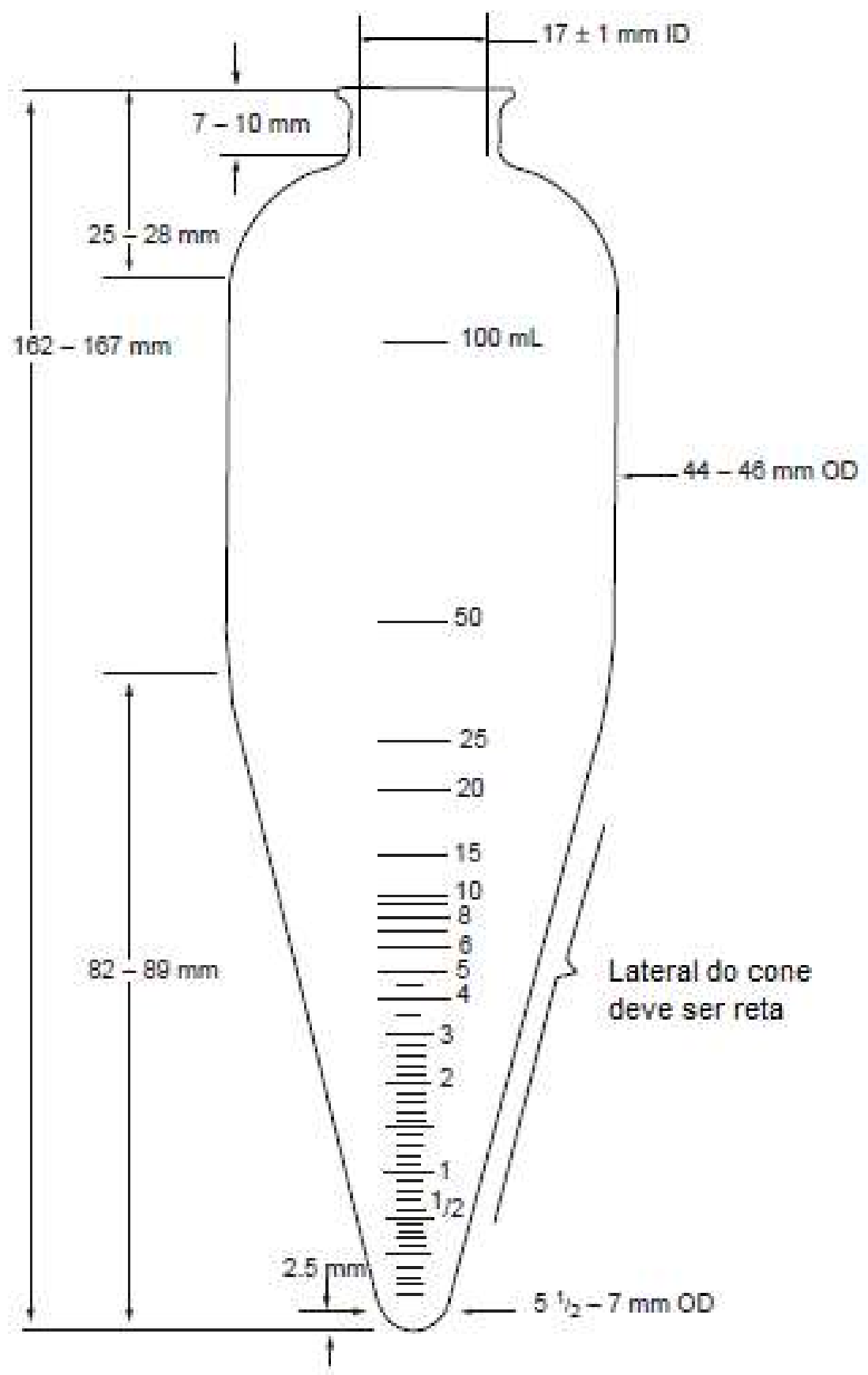

Figura 3 - Tubo de 167 mm. Figura adaptada do Guia API MPMS 10.4.

A norma também especifica que o termômetro utilizado na amostra deve ter intervalos de $1{ }^{\circ} \mathrm{C}$ ou menos, com incerteza de $\pm 0,5{ }^{\circ} \mathrm{C}$.

São especificados dois tipos de graduação nos tubos: $100 \mathrm{ml}$ e 200 partes. Para obter os resultados do método, utiliza-se a Tabela 3.

Por último, o guia não descreve alguma precisão, afirmando que este é um método de campo e que a experiência mostra que a determinação de teor de água utilizando este tem sido tão precisa como outros métodos de teste aceitos. 
Tabela 3 - Expressão de resultados para tubos de $100 \mathrm{ml}$ e de 200 partes, conforme guia API MPMS 10.4

\begin{tabular}{|c|c|c|c|c|}
\hline \multicolumn{2}{|c|}{$\begin{array}{c}\text { Tubo 100 ml } \\
\text { Volume de água e/ou } \\
\text { sedimentos (ml) }\end{array}$} & $\begin{array}{c}\text { Tolume de água e/ou } \\
\text { sedimentos (\%) }\end{array}$ & $\begin{array}{c}\text { Leitura do } \\
\text { total de água } \\
\text { e/ou } \\
\text { sedimentos }\end{array}$ \\
\hline Tubo 1 & Tubo 2 & Tubo 1 & Tubo 2 & $\mathbf{( \% )}$ \\
\hline$<\mathbf{0 , 0 1 2 5}$ & $<0,0125$ & $<0,025$ & $<0,025$ & 0,000 \\
\hline$<\mathbf{0 , 0 1 2 5}$ & 0,025 & $<0,025$ & 0,050 & 0,025 \\
\hline $\mathbf{0 , 0 2 5}$ & 0,025 & 0,050 & 0,050 & 0,050 \\
\hline $\mathbf{0 , 0 2 5}$ & 0,050 & 0,050 & 0,100 & 0,075 \\
\hline $\mathbf{0 , 0 5 0}$ & 0,050 & 0,100 & 0,100 & 0,100 \\
\hline $\mathbf{0 , 0 5 0}$ & 0,075 & 0,100 & 0,150 & 0,125 \\
\hline $\mathbf{0 , 0 7 5}$ & 0,075 & 0,150 & 0,150 & 0,150 \\
\hline $\mathbf{0 , 0 7 5}$ & 0,100 & 0,150 & 0,200 & 0,175 \\
\hline $\mathbf{0 , 1 0 0}$ & 0,100 & 0,200 & 0,200 & 0,200 \\
\hline $\mathbf{0 , 1 0 0}$ & 0,150 & 0,200 & 0,300 & 0,250 \\
\hline
\end{tabular}

\section{3}

\section{Método Karl Fischer}

A norma ASTM 10.7/2002 trata do Método Karl Fisher Volumétrico. A segunda edição é de dezembro de 2002, porém sofreu reafirmação em maio de 2011. Este método é utilizado para análise de água entre $0,02 \%$ e 2,00\% em volume para óleo cru que, depois de homogeneizado em um misturador com uso de um solvente, é titulado até um ponto final eletrométrico utilizando o reagente Karl Fischer, solução de éter monometílico de etileno glicol, estabilizada, contendo piridina ou isento de piridina diluído com xileno.

Algumas substâncias podem interferir no resultado analítico, principalmente as associadas à condensação e à óxido-redução. No óleo cru, as impurezas mais comuns são mercaptanas e sulfetos.

Este método utiliza os seguintes equipamentos: aparelho de Karl Fisher Volumétrico, misturador e seringas. 
Para amostragem, recomenda a utilização dos métodos ASTM D 4057 (API MPMS Ch. 8.1) e ASTM D 4177 (API MPMS CH 8.2).

Calibra-se o equipamento, inicialmente, adicionando solvente suficiente no vaso de titulação para cobrir os eletrodos. Vedam-se todas as aberturas do recipiente e inicia-se a agitação com uma frequência baixa. Liga-se o circuito de medição e ajusta-se o potenciômetro para indicar um ponto de referência de aproximadamente $1 \mu \mathrm{A}$ de corrente. Adiciona-se, então, o reagente de Karl Fischer em quantidades adequadas no solvente até que haja deflexão do ponteiro do amperímetro em relação ao ponto de referência. Inicialmente o ponteiro defletirá devido à concentração local do reagente não consumido sobre os eletrodos e, em seguida, retornará à proximidade do ponto de referência. Com a aproximação do ponto final, o ponteiro retornará mais lentamente ao ponto de referência depois de cada adição do reagente de Karl Fischer. O ponto final é alcançado quando o ponteiro deflete $1 \mu \mathrm{A}$ do ponto inicial durante pelo menos $30 \mathrm{~s}$ após a adição de uma única gota de reagente.

Em seguida, padroniza-se o reagente Karl Fischer com água destilada utilizando-se um dos seguintes métodos:

- Tomar a massa ao $0,1 \mathrm{mg}$ mais próximo de 1 gota de água destilada (cerca de $20 \mathrm{mg}$ ) a partir de uma pipeta ou seringa de pesagem cheia de água e adicioná-la ao solvente da amostra. Titular a água com reagente Karl Fischer adicionado da bureta até atingir um ponto final estável durante pelo menos 30 s. Registrar o volume do reagente Karl Fischer necessário para atingir o ponto final, com a aproximação de $0,01 \mathrm{~mL} ;$ ou

- $\quad$ Encher uma seringa de $10 \mu \mathrm{L}$ com água, tomando cuidado para eliminar bolhas de ar. Limpar a agulha com um lenço de papel para remover qualquer água residual e determinar a massa da seringa mais água ajustando para o $0,1 \mathrm{mg}$. Adicionar o conteúdo da seringa ao solvente da amostra na célula que foi ajustada para o ponto final garantindo que a ponta da agulha esteja abaixo da superfície do solvente. Fechar novamente o recipiente. Remover qualquer solvente da agulha limpando com um tecido de papel e tomar a massa novamente a seringa para $0,1 \mathrm{mg}$ mais próximo. Titula-se a água com o reagente Karl Fischer.

Para calcular a equivalência em água do reagente Karl Fischer, utiliza-se 


$$
F=\frac{W}{T}
$$

Sendo:

$F$ - água equivalente ao reagente Karl Fischer, em mg/ml;

$W$ - massa de água adicionada, em mg; e

$T$ - volume de reagente necessário para titulação, em $\mathrm{ml}$.

Após isto, registra-se o valor médio de equivalência em água.

O processo inicia-se com a adição de solvente na nova amostra dentro do recipiente de titulação, trazendo-a à condição de ponto final de solvente como já descrito. Adiciona-se, então, o óleo cru ao recipiente de titulação usando um dos seguintes métodos para análise da massa do teor de água na amostra:

a) Utilizando uma seringa limpa, retirar a quantidade necessária de amostra e descarregá-la no recipiente de titulação. Titular a amostra até um ponto final estável por pelo menos $30 \mathrm{~s}$. Registrar o volume de reagente de Karl Fischer com a aproximação de 0,01 ml;

b) Para óleos viscosos, utiliza-se um frasco conta-gotas limpo. Transferir rapidamente a quantidade necessária de amostra para o vaso de titulação com o conta-gotas. Titula-se a amostra.

Para calcular a quantidade de água existente na amostra, utiliza-se

$$
\text { massa de água em } \%=\frac{C * F}{W *(10)},
$$

Onde:

$C$ - volume de reagente Karl Fischer utilizado na titulação, em ml;

$F$ - água equivalente ao reagente Karl Fischer, em mg/ml; e

$W$ - massa de amostra utilizada, em $\mathrm{g}$.

A repetibilidade, $r$, excederá os valores das equações (5) para reagente Karl Fischer padrão e (6) para reagente livre de piridina em um caso em vinte, conforme Tabela 4.

$$
\begin{aligned}
& r=0,034\left(X_{v}^{\frac{1}{3}}\right) \text { ou } \\
& r=0,032\left(X_{v}^{\frac{1}{3}}\right)
\end{aligned}
$$

onde:

$X_{v}$ - média da amostra entre $0,0 \%$ e $2,0 \%$. 
A reprodutibilidade, $R$, só poderá exceder os valores das equações (7) para reagente Karl Fischer padrão e (8) para reagente livre de piridina em um caso em vinte, conforme Tabela 4.

$$
\begin{aligned}
& R=0,111\left(X_{v^{\frac{1}{3}}}\right), \\
& R=0,095\left(X_{v}^{\frac{1}{3}}\right),
\end{aligned}
$$

\begin{tabular}{|c|c|c|c|c|}
\hline \multirow{2}{*}{$\begin{array}{c}\text { Massa de } \\
\text { água } \\
(\%)\end{array}$} & \multicolumn{2}{|c|}{$\begin{array}{c}\text { Reagente Karl Fischer } \\
\text { Padrão }\end{array}$} & \multicolumn{2}{|c|}{ Reagente sem Piridina } \\
\hline & $\mathrm{r}$ & $\mathrm{R}$ & $\mathrm{r}$ & $\mathrm{R}$ \\
\hline 0,05 & 0,013 & 0,041 & 0,012 & 0,035 \\
\hline 0,1 & 0,016 & 0,052 & 0,015 & 0,044 \\
\hline 0,3 & 0,023 & 0,074 & 0,021 & 0,064 \\
\hline 0,5 & 0,027 & 0,088 & 0,025 & 0,075 \\
\hline 0,7 & 0,030 & 0,099 & 0,028 & 0,084 \\
\hline 1,0 & 0,034 & 0,111 & 0,032 & 0,095 \\
\hline 1,3 & 0,037 & 0,121 & 0,035 & 0,104 \\
\hline 1,5 & 0,039 & 0,127 & 0,037 & 0,109 \\
\hline 1,7 & 0,041 & 0,132 & 0,038 & 0,113 \\
\hline 2,0 & 0,043 & 0,140 & 0,040 & 0,120 \\
\hline
\end{tabular}

Tabela 4 - Precisão em cada intervalo, conforme Guia API MPMS 10.7.

A Resolução ANP número 52, de 26 de dezembro de 2013, aprova o Regulamento Técnico de Implementação de Resultados de Análises FísicoQuímicas para as Medições Subsequentes de Petróleo e Gás Natural. Este regulamento, em seu item 4.2.2.1, diz que podem ser utilizadas as seguintes normas para determinação da fração volumétrica de água e sedimentos, além das apontadas pelo RTM:

ASTM D4928/2012: Standard Test Method for Water in Crude Oils by Coulometric Karl Fischer Titration; ou

- $\quad$ API/MPMS 10.9/2013: Standard Test Method for Water in Crude Oils by Coulometric Karl Fischer Titration.

Este método aplica-se à determinação de água em óleo no intervalo de 0,02\% e 5,00\% em massa ou volume em óleo cru. 
Neste método, ao invés de se utilizar o titulante na seringa, utiliza-se um eletrodo na célula de titulação do aparelho para gerar o reagente através de uma corrente elétrica. Esta corrente gera o iodo para a reação a partir do iodeto contido no reagente anódico. Com base na estequiometria da reação, um mol de iodo reage com um mol de água. A titulação ocorre até que toda a água seja consumida e sobre uma quantidade residual de iodo sem reagir, o que gera um aumento abrupto de corrente, detectado pelo eletrodo indicador. $\mathrm{O}$ instrumento mede o fluxo de corrente e o tempo necessários para alcançar o fim da reação e calcula a quantidade de moléculas de água que reagiram. Esta quantidade é convertida em volume para o cálculo da fração volumétrica de água existente na amostra.

A precisão deste método de ensaio é criticamente dependente da eficácia da etapa de homogeneização. Além disso, se o método de ensaio é realizado em uma base de volume, a precisão é criticamente dependente da precisão e repetibilidade do volume injetado.

O cálculo da porcentagem em massa de água no óleo segue a equação (9) e deve ser aproximado para o valor mais próximo em $0,01 \%$ de massa. $O$ cálculo em volume segue a equação (10) e deve ser aproximado para o valor mais próximo em $0,01 \%$ em volume.

$$
\text { Massa de água, em } \%=\frac{W_{1}}{W_{2}} * 100,
$$

Sendo:

$W_{1}$ - massa de água titulada, em $\mu \mathrm{g}$; e

$W_{2}$ - massa da amostra, em $\mu \mathrm{g}$.

$$
\text { Volume de água, em } \%=\frac{V_{1}}{V_{2}} * 100 \text {, }
$$

Sendo:

$V_{l}$ - volume de água titulada, em $\mu 1$; e

$V_{2}$ - volume da amostra, em $\mu 1$.

A repetibilidade $(r)$ deste método deve seguir a equação (11) para massa e (12) para volume, e a reprodutibilidade $(R)$ deve seguir a equação (13) para massa e (14) para volume. Se os resultados da repetibilidade e reprodutibilidade forem inferiores aos valores da Tabela 5, a média dos dois deve ser o resultado relatado.

$$
r=0,040\left(X_{c}^{\frac{2}{3}}\right),
$$




$$
\begin{aligned}
& r=0,056\left(X_{c}^{\frac{2}{3}}\right), \\
& R=0,105\left(X_{c}^{\frac{2}{3}}\right) e \\
& R=0,112\left(X_{c}^{\frac{2}{3}}\right),
\end{aligned}
$$

sendo $X_{c}$ a média da amostra entre $0,02 \%$ e 5,00\%.

Tabela 5 - Precisão em cada intervalo, conforme Norma API MPMS 10.9.

ل)

\begin{tabular}{|c|c|c|c|c|}
\hline $\begin{array}{c}\text { \% de água } \\
\text { (Massa ou } \\
\text { Volume) }\end{array}$ & Massa & Volume & Massa & Volume \\
\hline $\mathbf{0 , 0 1}$ & 0,002 & 0,003 & 0,005 & 0,005 \\
\hline $\mathbf{0 , 0 2}$ & 0,003 & 0,004 & 0,008 & 0,008 \\
\hline $\mathbf{0 , 0 5}$ & 0,005 & 0,008 & 0,014 & 0,015 \\
\hline $\mathbf{0 , 1}$ & 0,01 & 0,01 & 0,02 & 0,02 \\
\hline $\mathbf{0 , 3}$ & 0,02 & 0,03 & 0,05 & 0,05 \\
\hline $\mathbf{0 , 5}$ & 0,03 & 0,04 & 0,07 & 0,07 \\
\hline $\mathbf{0 , 7}$ & 0,03 & 0,04 & 0,08 & 0,09 \\
\hline $\mathbf{1 , 0}$ & 0,04 & 0,06 & 0,11 & 0,11 \\
\hline $\mathbf{1 , 5}$ & 0,05 & 0,07 & 0,14 & 0,15 \\
\hline $\mathbf{2 , 0}$ & 0,06 & 0,09 & 0,17 & 0,18 \\
\hline $\mathbf{2 , 5}$ & 0,07 & 0,10 & 0,19 & 0,21 \\
\hline $\mathbf{3 , 0}$ & 0,08 & 0,12 & 0,22 & 0,23 \\
\hline $\mathbf{3 , 5}$ & 0,09 & 0,13 & 0,24 & 0,26 \\
\hline $\mathbf{4 , 0}$ & 0,10 & 0,14 & 0,26 & 0,28 \\
\hline $\mathbf{4 , 5}$ & 0,11 & 0,15 & 0,29 & 0,31 \\
\hline $\mathbf{5 , 0}$ & 0,12 & 0,16 & 0,31 & 0,33 \\
\hline
\end{tabular}

\section{4}

\section{Analisadores Automáticos de Teor de Água}

Segundo Costa e Silva (2013), os medidores de teor de água em linha existentes no mercado em 2013 podiam ser classificados como medidores de fração de água (Water Cut), pois não mediam os sedimentos presentes no óleo. Afirma, também, que a principal vantagem na utilização deste tipo de medidor é obter os valores do teor de água em tempo real e não por amostragem, tendo como 
desvantagem a necessidade de calibração periódica do equipamento, definida pelo RTM como 6 meses para pontos fiscais e 9 meses para pontos de apropriação (pontos de medição utilizados para apropriar a produção a cada poço e para divisão dos royalties entre os devidos municípios) e transferência de custódia (pontos de medição utilizados para transferência legal e/ou comercial de fluidos hidrocarbonetos).

O RTM, em seu item 7.1.7, afirma que o teor de água deve ser proporcional à vazão. $\mathrm{O}$ mesmo define que este deve ser abaixo de $1 \%$ em volume nos pontos fiscais, mas não define faixas para pontos de apropriação ou operacionais. A incerteza de medição permitida para a malha de óleo fiscal $(0,3 \%)$ é mais restrita do que para a malha de apropriação $(1,0 \%)$, portanto deve-se utilizar medidores com menor incerteza para atender a faixa de 0 a $1 \%$.

Ao longo dos anos, uma série de tecnologias foram desenvolvidas com diferentes graus de sucesso para medição de teor de água, havendo os seguintes princípios de medição utilizados em instrumentos disponíveis no mercado: pressão diferencial, sensor mássico por efeito coriolis, infravermelho, gama densiômetro, sensor capacitivo, micro-ondas e ultrassom (Liu, 2006). Por último, este autor cita que nos últimos anos tem-se registrado um desenvolvimento significativo nesta área, tendo os fabricantes desenvolvido um grau de maturidade na aplicação de variadas técnicas de fabricação de medidores para fração de água. . 


\section{3 Planejamento de Experimentos}

\section{1 Definição}

Em um experimento, os fatores influentes podem agir de forma independente uns dos outros ou em conjunto. Considerando um caso em que apenas dois fatores influenciam um dado resultado, nem sempre fixar um fator e variar o outro até sua otimização e repetir a operação invertendo os fatores leva à otimização da resposta do experimento. Neto et al. (2003) afirmam que, ao contrário do senso comum, é possível obter melhores resultados ao se estudar o sistema variando todos os fatores ao mesmo tempo.

O planejamento de experimentos é uma ferramenta estatística importante para extrair o máximo de informação com um menor número de iterações, ajudando a atingir o objetivo mais rapidamente e com um menor custo.

O planejamento de experimentos visa projetar um experimento de forma que o mesmo venha a fornecer os dados necessários para obter o resultado procurado. Para isto, o primeiro passo é selecionar quais variáveis influenciam neste, descartando variáveis não significativas. Após isto, avalia-se quantitativamente a influência das variáveis selecionadas sobre a resposta. Definem-se, então, os critérios a serem seguidos (condições de contorno, se a resposta será maximizada ou minimizada, a variação máxima permitida para cada variável, etc.). Constrói-se, então, um modelo para análise do sistema.

Neto et al. (2003) enfatizam que somente construir este modelo não basta, é necessário avaliar se o mesmo é adequado ou não ao experimento estudado. Somente tendo esta certeza será possível extrair respostas satisfatórias com a análise deste modelo e seus resultados por meio das ferramentas estatísticas apropriadas.

Ou seja, o planejamento de experimentos permite observar quais variáveis exercem maior influência nas respostas de um sistema e se há interação entre estas variáveis. 


\section{2 \\ Planejamento Fatorial}

Segundo Cunico et al. (2008), o planejamento fatorial permite avaliar simultaneamente o efeito de diversas variáveis utilizando um número reduzido de ensaios, sem prejudicar a qualidade da informação, quando comparado a outros métodos dentro do planejamento de experimentos. Esta ferramenta permite que, em cada tentativa, sejam investigadas várias das combinações possíveis dos níveis de cada variável, determinando-se o erro experimental e a variabilidade do experimento. Segundo os autores, outra vantagem é o fato de que a pesquisa pode ser realizada de forma iterativa, em etapas, acrescentando novos ensaios à medida que a mesma é analisada.

Neto et al. (2003) definem os fatores como as variáveis a serem estudadas. Em seguida, dizem que é necessário especificar os níveis em que cada fator será estudado, ou seja, seus valores ou classes. Por exemplo, pode-se fazer um estudo em relação à variação da pressão, sendo esta estudada em três níveis: $20 \mathrm{kPa}, 30 \mathrm{kPa}$ e $40 \mathrm{kPa}$. Define-se, então, o modelo estatístico que irá avaliar os efeitos dos fatores sobre a resposta do sistema.

Segundo Neves et al. (2002, apud Cunico et al., 2008), pode-se representar um planejamento fatorial como $b^{k}$, sendo

- $\quad b$ o número de níveis escolhidos; e

- $\quad k$ o número de fatores.

Ambos os artigos citados apresentam o caso $2^{k}$ (dois níveis) como o mais simples. Porém, segundo Neves et al. (2002, apud Cunico et al., 2008), mesmo sendo impossível explorar completamente uma grande região no espaço das variáveis, este tipo de planejamento ainda se mostra vantajoso, pois é possível avaliar importantes tendências do caso investigado.

Cunico et al. (2008) definem como efeito a mudança ocorrida na resposta quando se muda o nível estudado. Definem o nível baixo ou inferior (-) e o nível alto ou superior $(+)$. A utilização destes sinais permite utilizar as variáveis na forma de matrizes de planejamento, que serão úteis para estudar as interações das variáveis com o sistema. Ou seja,

$$
\text { Efeito }=\overline{R_{+}}-\overline{R_{-}} \text {, }
$$

sendo: 
$\overline{R_{+}}$- média das respostas obtidas com os valores superiores $(+)$do fator; e

$\overline{R_{-}}$- média das respostas obtidas com os valores inferiores (-) do fator.

Cunico et al. (2008) definem, ainda, o efeito principal como a diferença média observada na resposta com as mudanças de nível dos fatores, ou seja,

$$
\text { Efeito Principal }=2\left(\sum y^{+}-\sum y^{-}\right) /\left(b^{x}\right),
$$

sendo:

$y$ - média dos efeitos na medida; e

$b^{k}$ - número total de experimentos.

Neste caso, o erro padrão do efeito pode ser calculado por

$$
\text { Erro Padrão do efeito }=\frac{s}{\sqrt{2^{k-1}}}
$$

sendo:

$s$ - desvio-padrão da medida; e

$k$ - número de fatores experimentais utilizados.

Os autores finalizam afirmando que é esperado que o erro nos efeitos seja menor que os erros das medidas, por utilizarem valores médios em seu cálculo.

\section{3 \\ Superfície de Resposta}

A superfície de resposta é uma técnica de otimização baseada em planejamento fatorial que corresponde a um gráfico tridimensional que auxilia visualmente na busca do ponto ótimo (Oliveira, 2006).

Uma superfície de resposta teórica que expressa a relação entre a resposta esperada e as variáveis 1 e 2 está representada na Figura 4. Como complemento, pode-se construir um gráfico de contorno da superfície de resposta, ou curvas de nível, no plano Variável 1-Variável 2. 


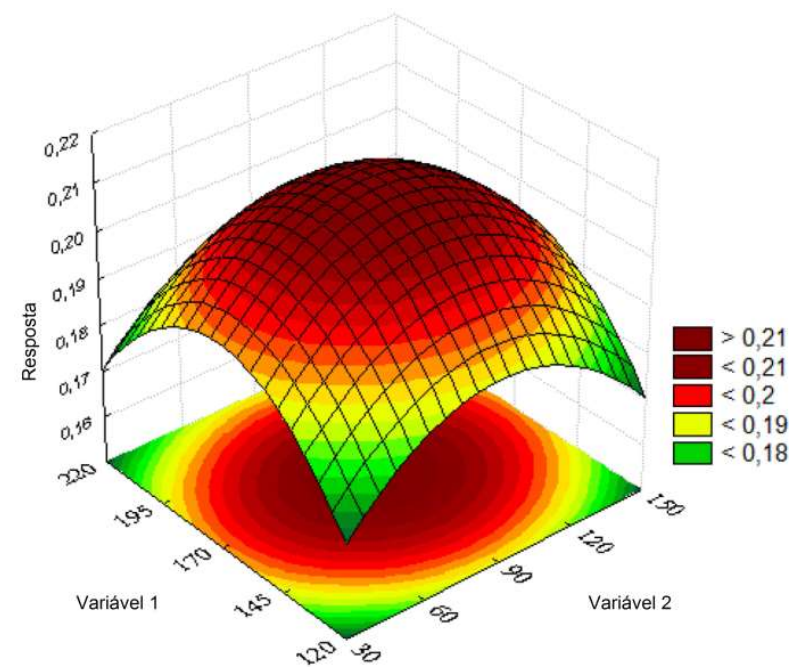

Figura 4 - Exemplo de superfície de resposta teórica aplicada a um processo químico.

Observa-se que, para cada par de valores das variáveis 1 e 2, tem-se um valor correspondente para a resposta. Estes valores geram uma superfície situada acima do plano Variável 1-Variável 2 (Myers et al., 2016). No ápice da superfície (ponto de máximo), tem-se a otimização do processo.

Neto et al. (2003) afirmam que esta metodologia, também chamada de RSM, Response Surface Methodology, tem duas etapas:

- Modelagem - ajuste de modelos simples, em geral lineares ou quadráticos, às respostas obtidas utilizando planejamento fatorial; e

- Deslocamento - movimento pelo modelo na direção de máxima inclinação.

As etapas podem ser repetidas quantas vezes forem necessárias até atingir-se a otimização procurada.

O caminho de máxima inclinação para uma superfície de resposta determinada por $k$ fatores pode ser calculado algebricamente a partir dos coeficientes do modelo adotado. É proporcional aos módulos e sinais do coeficiente do modelo.

Neto et al. (2003) exemplificam considerando um modelo simples e linear, com dois fatores, $x_{1}$ e $x_{2}$,

$$
\hat{y}=b_{0}+b_{1} x_{1}+b_{2} x_{2}
$$

onde $b_{0}, b_{1}$ e $b_{2}$ são os estimadores dos parâmetros do modelo e podem ser obtidos pelo método dos mínimos quadrados. Neste caso, a máxima inclinação é calculada fazendo deslocamentos no eixo $x_{1}$ e $x_{2}$ na proporção $b_{2} / b_{1}$. 
Neto et al. (2003) descrevem o seguinte procedimento para calcular este caminho em um caso geral:

- $\quad$ Escolhe-se um dos fatores como base, aqui descrito como $i$;

- $\quad$ Muda-se o nível do fator $i$ em uma determinada extensão para mais e para menos, conforme o objetivo do experimento, obtendo um ponto máximo e um ponto mínimo da resposta. O seu deslocamento é de uma unidade na escala codificada, como será explicado mais à frente;

- Deslocam-se os outros fatores diferentes de $i$, aqui definido um fator $j$, em unidades codificadas, obtendo:

$$
\Delta x_{j}=\frac{b_{j}}{b_{i}} \Delta x_{i}
$$

- Obtêm-se as unidades originais convertendo os deslocamentos codificados e determinando os novos níveis dos fatores.

Segundo o autor, há autores que recomendam que a primeira variável a ser escolhida seja a de maior coeficiente, em módulo.

Pode-se exemplificar as unidades codificadas com um experimento em que o teor de água e a temperatura são estudados. Neste caso, o teor de água médio de operação é $1,0 \%$ e a temperatura $60^{\circ} \mathrm{C}$.

O nível baixo (-) do teor de água foi definido por meio de estudos como $0,5 \%$ e o nível alto (+) como $1,5 \%$. No caso da temperatura, $50{ }^{\circ} \mathrm{C}$ e $70{ }^{\circ} \mathrm{C}$, respectivamente. Os fatores codificados poderiam seriam representados por:

$$
x_{1}=\frac{\text { variável }-1,0}{1,5-0,5} \text { e } x_{2}=\frac{\text { temperatura }-60}{70-50},
$$

O autor afirma, ainda, que a escolha da faixa inicial de variação dos fatores é de grande importância, pois dela é consequência direta o tamanho do primeiro planejamento, a escala de codificação e a velocidade de deslocamento dos experimentos seguintes.

\section{4}

\section{Aplicação em um modelo quadrático}

Para construir a matriz experimental de um caso quadrático utilizando a técnica de planejamento fatorial composto central, que consiste em acrescentar pontos centrais e axiais ao experimento fatorial completo, de forma que sejam 
apresentados os níveis de ajuste dos fatores de controle em termos de variáveis codificadas, utiliza-se a seguinte equação:

$$
y=b_{0}+b_{1} * x_{1}+b_{2} * x_{2}+b_{3} * x_{1} * x_{2}+b_{4} * x_{1}^{2}+b_{5} * x_{2}^{2},
$$

sendo $x_{1}$ e $x_{2}$ os fatores de maior influência no experimento.

Para encontrar os coeficientes $b_{i}$, podem-se dispor os valores em matrizes, neste exemplo utilizando seis amostras, sendo:

$$
y=\left|\begin{array}{l}
y_{1} \\
y_{2} \\
y_{3} \\
y_{4} \\
y_{5} \\
y_{6}
\end{array}\right|
$$

$$
x=\left|\begin{array}{cccccc}
1 & x_{11} & x_{21} & x_{11} * x_{21} & x_{11}^{2} & x_{21}^{2} \\
1 & x_{12} & x_{22} & x_{12} * x_{22} & x_{12}^{2} & x_{22}^{2} \\
1 & x_{13} & x_{23} & x_{13} * x_{23} & x_{13}^{2} & x_{23}^{2} \\
1 & x_{14} & x_{24} & x_{14} * x_{24} & x_{14}^{2} & x_{24}^{2} \\
1 & x_{15} & x_{25} & x_{15} * x_{25} & x_{15}^{2} & x_{25}^{2} \\
1 & x_{16} & x_{26} & x_{16} * x_{26} & x_{16}^{2} & x_{26}^{2}
\end{array}\right| \mathrm{e}
$$

$$
b=\left|\begin{array}{l}
b_{0} \\
b_{1} \\
b_{2} \\
b_{3} \\
b_{4} \\
b_{5}
\end{array}\right| .
$$

Os coeficientes $b$ podem ser calculados utilizando o seguinte cálculo matricial:

$$
b=\left(x^{\mathrm{t}} x\right)^{-1} x^{\mathrm{t}} y
$$

Utilizando a superfície de resposta, localiza-se o ponto ótimo $x_{\mathrm{o}}\left(x_{10}, x_{2 \mathrm{o}}\right)$. Partindo do ponto central, utiliza-se a equação a seguir para transformar as variáveis codificadas em variáveis verdadeiras:

$$
\text { Fator }=\text { ponto central }+\left(x_{\mathrm{o}} * \text { variação }\right) \text {, }
$$

Neto et al. (2003) afirmam que, caso o ponto máximo não fosse atingido, e sim encontrado um ponto de mínimo, deve-se deslocar novamente seguindo o caminho de máxima inclinação, repetindo o processo de modelagem e deslocamento quantas vezes forem necessárias até se atingir o ponto ótimo necessário. 


\section{5}

\section{Planejamento Composto Central}

O Planejamento Composto Central, ou Central Composite Design (CDD) é um tipo de planejamento em que o número de experimentos $n$, considerando $k$ variáveis ou fatores, é calculado por $2^{k}+2 * k+1$ e cuja robustez se baseia em sua constituição por um planejamento fatorial em dois níveis, por experimentos realizados no ponto central e em pontos axiais, $\alpha$. (Lemos et al., 2012).

Este tipo de planejamento tem como vantagem necessitar de um menor número de ensaios. Para executá-lo, define-se o número de variáveis $k$ a serem estudadas e quantos experimentos serão realizados no ponto central e nos pontos axiais. (Teófilo \& Ferreira, 2006). Para isso, codificam-se os níveis axiais a partir dos valores experimentais utilizando

$$
x_{i}=\frac{z_{i}-\bar{z}}{\frac{\Delta z}{2}}
$$

sendo:

$x_{i}$ - valor da variável codificada;

$z_{i}$ - valor da variável experimental;

$\bar{Z}$ - valor médio do nível codificado, podendo ser mais (+) ou menos (-);

$\Delta z$ - diferença entre os níveis mais (+) e menos (-).

No ponto central, considera-se $x_{i}$ igual a zero e nos pontos axiais $x_{i}= \pm \alpha$. A Figura 5 exemplifica um planejamento com três variáveis em dois níveis.

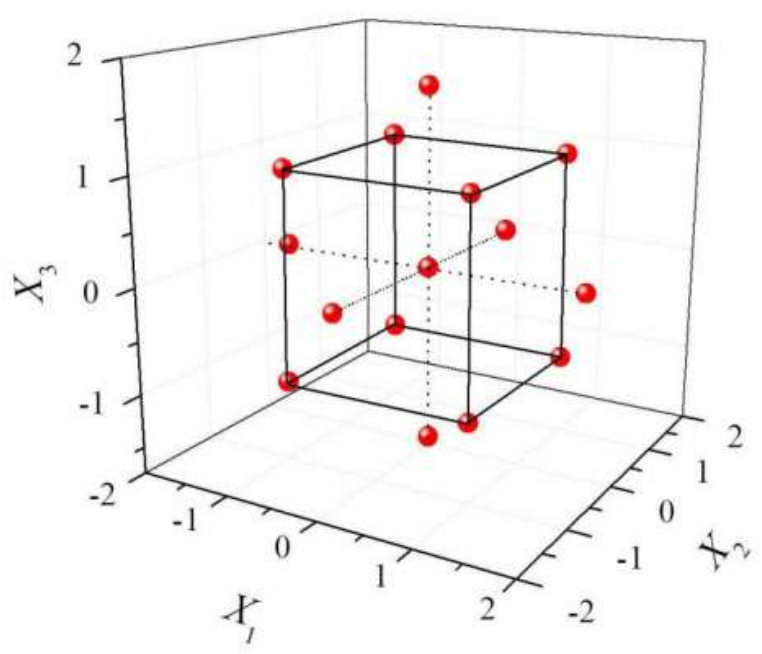

Figura 5 - Exemplo de Planejamento Composto Central com três variáveis em dois níveis (Lemos et al., 2012). 
Este tipo de planejamento permite avaliar de forma eficiente os coeficientes e o modelo por meio da regressão feita e proporciona boas estimativas para os coeficientes. É a classe de planejamento mais utilizada para ajustar modelos quadráticos, utilizando a equação (28), e apresenta vantagens como rotabilidade (homogeneidade da variância em qualquer direção) e blocagem ortogonal, que demonstra a independência entre as variáveis (Teófilo \& Ferreira, 2006).

$$
y=b_{0}+\sum_{i=1}^{k} b_{i} x_{i}+\sum_{i=1}^{k} b_{i i} x_{i}^{2}+\sum_{i<j} \sum_{j} b_{i j} x_{i} x_{j}+e .
$$

$\mathrm{Na}$ Tabela 6 tem-se um exemplo da matriz de planejamento composto central com três variáveis (A, B e C). Por meio desta é possível observar as três partes deste planejamento: a parte fatorial, que contém os pontos de coordenada \pm 1 ; o ponto central, com coordenadas 0 ; e a parte axial, com duas coordenadas nulas e uma com o valor $\pm \alpha$.

Tabela 6 - Matriz de Planejamento Composto Central com três variáveis.

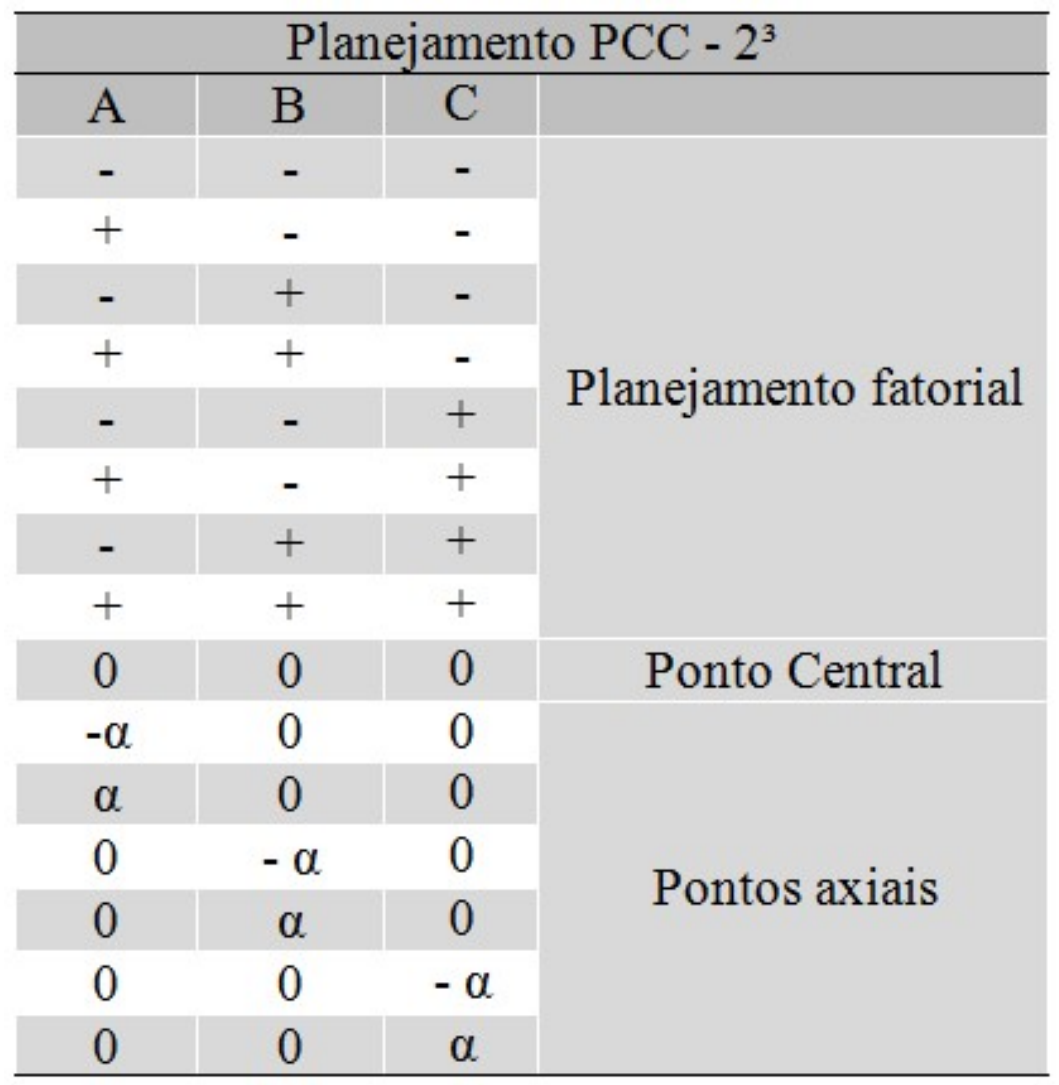

Pode-se gerar um experimento em que os valores axiais sejam $\pm \alpha=1$. Neste caso, tem-se um experimento centralizado na face (faced-centred).

Nesta dissertação, o CDD utilizará um planejamento fatorial completo $\left(2^{k}\right)$, podendo utilizar o cálculo do número de experimentos apresentado por Lemos et 
al. (2012). Após a realização dos experimentos, os dados serão avaliados por superfície de resposta até encontrar os valores que levem à região ótima desta. 


\section{4 \\ Descrição e Incerteza da Malha}

\section{1}

\section{Medição de Vazão de óleo pelo Princípio Ultrassônico}

Uma malha de controle é definida como um conjunto de instrumentos e equipamentos que têm por finalidade medir e controlar uma determinada variável (Dunn, 2013). As malhas de controle podem ser classificadas como abertas, caso em que as variáveis de saída não são utilizadas para controlar as variáveis de entrada; ou fechadas, caso em que há realimentação, ou seja, as variáveis de saída influenciam as variáveis controladas. O caso estudado nesta dissertação é uma malha de controle aberta de vazão.

Os medidores de vazão do tipo ultrassônico existentes no mercado utilizam três técnicas distintas como princípio de funcionamento: efeito Doppler, correlação cruzada e tempo de trânsito (Arantes, 2007). A técnica mais utilizada na indústria de petróleo que é discutida neste estudo é o tempo de trânsito.

A medição de vazão por meio deste terceiro princípio, ilustrada na Figura 6, considera a diferença entre as velocidades de uma onda acústica (ondas de pressão longitudinais, também chamadas de sinal ultrassônico) no sentido do escoamento, no qual esta será mais rápida, e no sentido contrário, no qual será mais lenta (Arantes, 2007). Visto que esta diferença de tempo é função da velocidade do fluido, a vazão volumétrica pode ser determinada pelo produto da área da seção transversal pela velocidade axial média do fluido.

Os sinais ultrassônicos são transmitidos e recebidos por transdutores fabricados com cristais piezelétricos que vibram, oscilando mecanicamente, e emitem ondas quando se aplica uma tensão elétrica nos mesmos. O efeito contrário também ocorre, emitindo tensão ao receber sinais acústicos (Santos, 2011). 


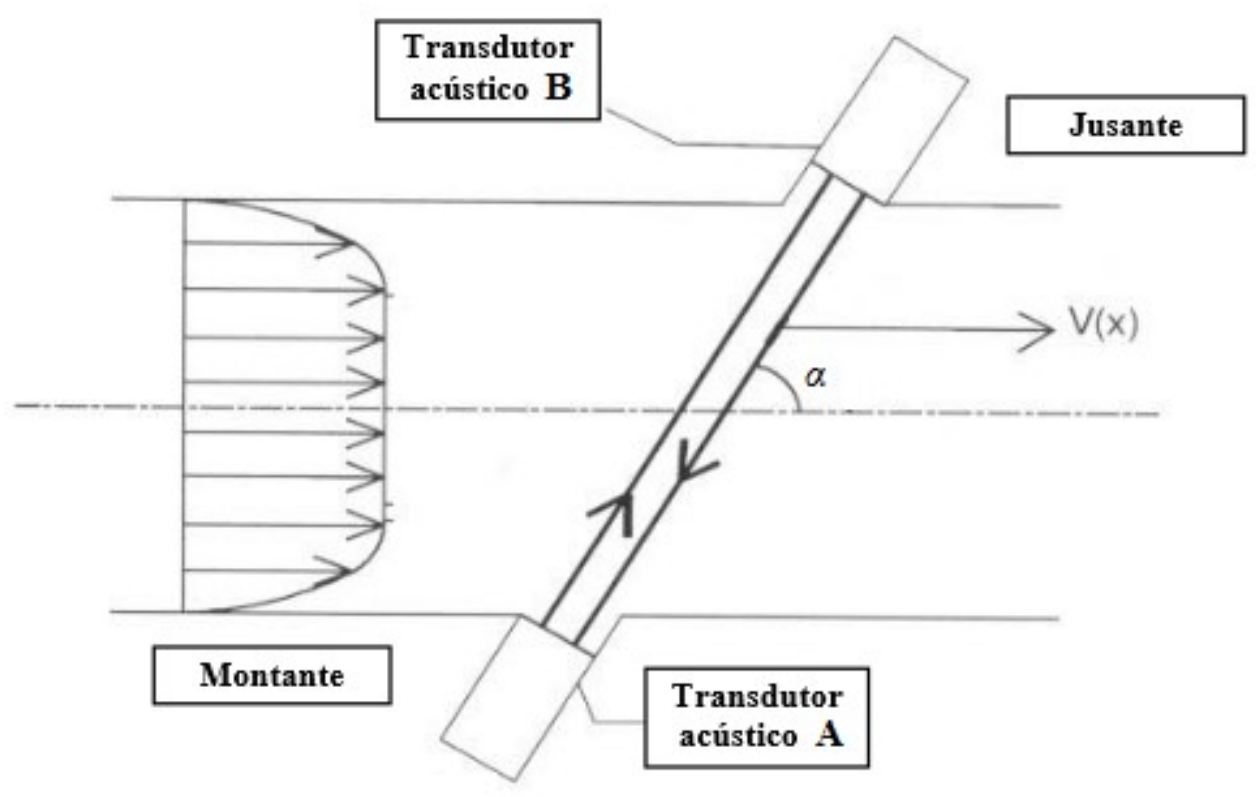

Figura 6 - Princípio de funcionamento dos medidores ultrassônicos por tempo de trânsito. Figura adaptada de Arantes, 2007.

O espectro de frequência emitido pelos cristais piezelétricos é classificado como onda ultrassônica, pois se encontra na faixa acima de $20 \mathrm{kHz}$, ou seja, acima do espectro do som audível (Salgado, 2009).

Os medidores ultrassônicos podem utilizar um (monocanal) ou mais pares de transdutores (multicanal). Salgado (2009) exemplifica em seu estudo um medidor monocanal supondo dois transdutores, A e B, com o fluxo do fluido na direção de A para B. Em um primeiro momento, o pulso avança no sentido do escoamento, gerando o tempo de trânsito a favor do fluxo $\left(t_{A B}\right)$, o qual retorna posteriormente no sentido contrário $\left(t_{B A}\right)$. Pode-se, então, determinar a velocidade da onda utilizando

$$
\begin{gathered}
V_{A B}=\frac{L}{t_{A B}}=c+V \cos \propto \mathrm{e} \\
V_{B A}=\frac{L}{t_{B A}}=c-V \cos \propto,
\end{gathered}
$$

sendo:

$L$ - distância entre os transdutores, ou caminho acústico;

$c$ - velocidade de propagação do som no fluido em escoamento;

$V$ - velocidade média ao longo do caminho acústico;

$\alpha$ - ângulo entre a linha reta que liga os transdutores, conhecida por visada direta, e a direção do escoamento.

Utilizando como base as equações (29) e (30), pode-se calcular 


$$
\begin{aligned}
& V=\frac{D}{\sin 2 \propto \frac{t_{A B}-t_{B A}}{t_{A B} * t_{B A}} \mathrm{e}} \\
& c=\frac{D}{\sin 2 \propto \frac{t_{A B}+t_{B A}}{t_{A B} * t_{B A}}},
\end{aligned}
$$

sendo D o diâmetro interno do duto.

No entanto, a velocidade $V$ calculada ainda não é a velocidade média necessária para o cálculo da vazão, sendo conhecida como bulk velocity, pois o perfil de velocidade do fluido na maioria das vezes não é uniforme, podendo apresentar assimetrias, vórtices e perfís diferenciados, dificultando o cálculo desta (Salgado, 2009).

\section{2}

\section{Sistema de Medição}

As malhas de medição de óleo, com finalidade fiscal, que servem de base para os dados desta dissertação, são compostas por um medidor primário de vazão do tipo ultrassônico e dois medidores secundários, de temperatura e pressão estática, utilizados para efetuar as correções na vazão medida para a condição padrão. Os instrumentos são interligados a um computador de vazão, que efetua os cálculos e correções utilizando as tabelas 53A e 54A da norma API MPMS Chapter 11.1 (2012).

O RTM define Computador de Vazão como:

"Dispositivo eletrônico, capaz de receber sinal de um medidor de vazão e demais dispositivos associados, de uma medição efetuada em determinadas condições de escoamento, e efetuar os cálculos necessários para que este valor de vazão seja convertido à condição padrão de medição".

Um exemplo simplificado desta malha de medição pode ser visto na Figura 7, que mostra o sentido do fluxo de vazão, os transdutores pertencentes ao instrumento primário, o medidor de vazão ultrassônico, o ângulo $\alpha$ entre a linha reta que liga os transdutores e a linha central da vazão e, por último, a representação dos medidores secundários de temperatura e pressão. 


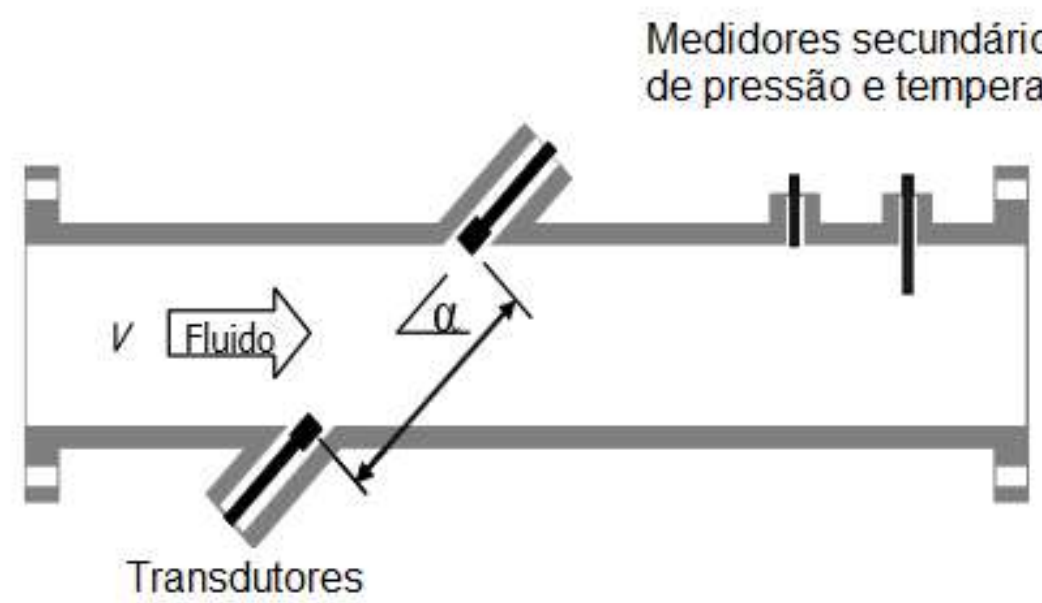

Figura 7 - Exemplo de malha de vazão utilizando o princípio ultrassônico (Salgado, 2009)

As vazões de petróleo estabilizado com BSW menor que $1 \%$ em volume, situação usual da malha estudada, podem ser calculadas por (API MPMS Chapter $11.1,2012)$

$$
\mathrm{Q}_{\mathrm{b}}=\text { MF. CTL.CPL. Q.SF. }(1-\text { BSW), }
$$

sendo:

$Q_{b}$ - Vazão nas condições padrão;

$Q$ - Vazão nas condições de operação;

$S F$ - Fator de encolhimento do óleo devido à liberação de gases e vapores;

$C T L$ - Fator de conversão do volume medido de óleo devido à diferença entre a temperatura de operação e a temperatura padrão;

$C P L$ - Fator de conversão do volume medido de óleo devido à diferença entre a pressão estática de operação e a pressão estática padrão.

O termo $M F$ da equação (33) é conhecido como meter factor, ou fator do medidor. O RTM apresenta a seguinte definição para este fator:

"Quociente entre o volume bruto medido, utilizando um medidor padrão de trabalho ou padrão de referência, e o volume medido por um medidor em operação durante uma calibração, sendo ambos referidos às mesmas condições de temperatura e pressão, ou ainda o quociente entre o volume bruto medido, utilizando um padrão de referência, e o volume medido por um medidor padrão de trabalho durante uma calibração, sendo ambos referidos às mesmas condições de temperatura e pressão."

Ou seja, o $M F$ é obtido por meio de um processo de calibração e deve ser aplicado para corrigir o valor medido pelo instrumento primário. A diferença entre os fatores de medição entre três testes consecutivos, dentro de uma calibração, deve ser menor que $0,05 \%$. O RTM define que a calibração destes medidores, para fins fiscais, deve ser feita semestralmente. 
O RTM define, ainda, que o medidor padrão de trabalho deve ser calibrado anualmente, em comparação com um provador padrão ou tanque, em laboratório acreditado pelo Cgcre (Coordenação Geral de Acreditação do INMETRO) e com diferença entre os fatores de medição entre testes consecutivos menor que 0,02\% (dois testes para tanque e cinco ou seis testes para provador) para os fatores do medidor.

Por último, este Regulamento define que, para medições fiscais de óleo, a variação do fator de calibração do medidor em relação ao da calibração imediatamente anterior não pode ultrapassar $0,25 \%$.

A API MPMS Chapter 11.1 (2012) também define as condições padrão, como sendo $20^{\circ} \mathrm{C}$ e $101,325 \mathrm{kPa}$. O RTM define este conjunto de temperatura e pressão como "Condição Padrão de Medição". A vazão calculada pelo computador de vazão, a qual será utilizada para o cômputo do volume exportado e para o pagamento de royalties e impostos para a União, é expressa em metros cúbicos por hora $\left(\mathrm{m}^{3} / \mathrm{h}\right)$ e apresentada nesta condição padrão.

O $C T L$ e o $C P L$ são calculados pelo computador de vazão utilizando a diferença entre a temperatura e pressão de operação, enviadas pelos medidores secundários, e as respectivas condições padrão. A vazão $Q$ é medida diretamente pelo medidor primário. O fator de encolhimento, $S F$, é obtido por meio de análise laboratorial do óleo produzido.

O $B S W$ (nesta dissertação, somente o teor de água) pode ser obtido em análise laboratorial ou por instrumento em linha.

\section{3}

\section{Incerteza da Malha Fiscal}

O item 10.1.14 do RTM menciona que todos os resultados de medições devem ter suas incertezas declaradas. O item 5.4.6 deste mesmo Regulamento define que o cálculo da incerteza de medição deve ser realizado após cada calibração de qualquer um dos instrumentos da malha, primário ou secundário, e que este deve passar por análise crítica e validação. Como já exposto nos capítulos anteriores, para uma malha com fins fiscais a incerteza de medição deve ser menor que $0,3 \%$.

A incerteza padrão combinada desta malha apresentada, considerando o modelo matemático descrito pela equação (33), considerando as recomendações de 
cálculos de incerteza do ISO GUM (International Organization for Standardization, 1995) e do EURACHEM (2000), pode ser calculada conforme

$$
\begin{aligned}
& u\left(Q_{m}\right)
\end{aligned}
$$

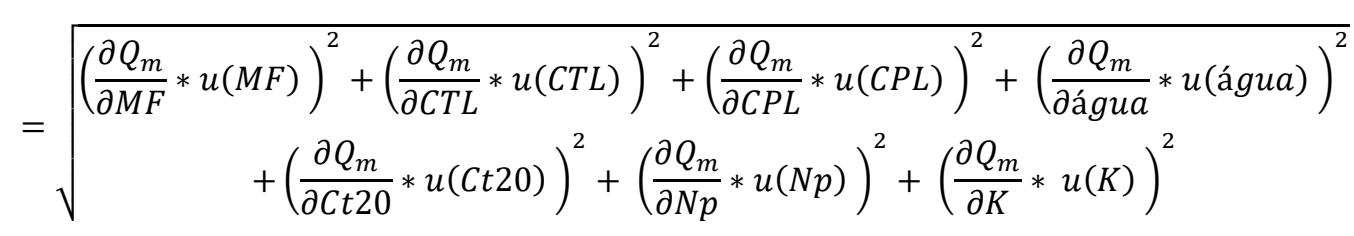

considerando:

$u\left(Q_{m}\right)$ - incerteza padrão combinada da malha;

$u(M F)$ - incerteza padrão do $M F$ obtido na calibração do mesmo (incerteza expandida declarada na última calibração dividida pelo fator de abrangência " $k$ " apresentado);

$u(C T L)$ - incerteza padrão indicada no último certificado de calibração do medidor de temperatura para a faixa na qual se enquadra a temperatura média de operação da malha;

$u(C P L)$ - incerteza padrão indicada no último certificado de calibração do medidor de pressão para a faixa equivalente à pressão média de operação da malha;

$u(C t 20)$ - incerteza padrão da determinação da massa específica para $20^{\circ} \mathrm{C}$, proveniente da análise laboratorial do óleo no dia da calibração do medidor (incerteza expandida no resultado dividida pelo fator de abrangência $k$, assumido como raiz de 3, distribuição retangular, nos casos em que não é definido);

$u(N p)$ - incerteza padrão do número de pulsos do medidor durante o período de calibração;

$u(K)$ - incerteza padrão na razão entre o número de pulsos do medidor e o volume computado. $\mathrm{O}$ fator $K$ nominal do medidor é o responsável pela conversão de pulsos em volume;

$u(a ́ g u a)$ - incerteza padrão associada à determinação do teor de água no óleo (em \% de volume por volume). No caso desta dissertação, associada ao resultado da análise emitida pelo laboratório no dia da calibração do medidor. Nos casos em que se utilizam analisadores em linha, deve-se utilizar as incertezas de calibração destes. Em ambos os casos, utiliza-se a incerteza expandida dividida pelo fator de abrangência " $k$ ", assumido com o valor raiz de 3, distribuição retangular, caso este não seja especificado.

A incerteza expandida da malha será calculada utilizando 


$$
U=k\left(0,9545 ; v_{\mathrm{eff}}\right) \cdot u_{\mathrm{malha}}
$$

sendo

$U$ - incerteza expandida de medição; e

$k$ - fator de abrangência com $v_{\text {eff }}$ graus de liberdade e nível de confiança de 0,9545 .

O ponto de partida adotado para esta dissertação foi a média da influência de cada parcela da incerteza de medição em seis medidores do tipo ultrassônicos utilizados em plataformas offshore, na função de medidor de óleo fisscal. Estes serão identificados, doravante, como Malha de 1 a 6.

Os cálculos de incerteza utilizados foram produzidos entre 2012 e 2015, conforme indicado na Tabela 7. Considerou-se que os dados de todas as parcelas seguem uma distribuição de probabilidade Normal. Observa-se que unicamente na Malha 4 a influência da incerteza do fator do medidor é maior que a do teor de água.

Tabela 7 - Influência de cada fator na incerteza das malhas estudadas, de 1 a 6 .

\begin{tabular}{|c|c|c|c|c|c|c|}
\hline $\begin{array}{c}\text { INCERTEZA } \\
\text { PADRÃO } \\
\mathbf{u}(\mathbf{x i})\end{array}$ & Malha 1 & Malha 2 & Malha 3 & Malha 4 & Malha 5 & Malha 6 \\
\cline { 2 - 7 } & Média & Média & Média & Média & Média & Média \\
\hline $\mathbf{u}(\mathbf{M F )}$ & $26,95 \%$ & $40,40 \%$ & $43,69 \%$ & $52,43 \%$ & $38,93 \%$ & $31,24 \%$ \\
\hline $\mathbf{u}(\mathbf{C T L})$ & $7,42 \%$ & $6,65 \%$ & $4,50 \%$ & $3,13 \%$ & $8,95 \%$ & $8,97 \%$ \\
\hline $\mathbf{u}(\mathbf{C P L})$ & $0,45 \%$ & $0,48 \%$ & $0,28 \%$ & $0,30 \%$ & $0,52 \%$ & $1,21 \%$ \\
\hline $\mathbf{u}($ água) & $65,13 \%$ & $52,42 \%$ & $51,50 \%$ & $44,12 \%$ & $51,57 \%$ & $58,53 \%$ \\
\hline $\mathbf{u}(\mathbf{C t 2 0})$ & $0,02 \%$ & $0,03 \%$ & $0,01 \%$ & $0,02 \%$ & $0,02 \%$ & $0,01 \%$ \\
\hline $\mathbf{u ( N p )}$ & $0,03 \%$ & $0,02 \%$ & $0,02 \%$ & $0,01 \%$ & $0,01 \%$ & $0,01 \%$ \\
\hline $\mathbf{u}(\mathbf{K})$ & $0,00 \%$ & $0,00 \%$ & $0,00 \%$ & $0,00 \%$ & $0,00 \%$ & $0,00 \%$ \\
\hline Amostras & 23 & 26 & 24 & 25 & 21 & 22 \\
\hline
\end{tabular}

Esta tabela será a base dos experimentos feitos no próximo capítulo.

\section{4}

\section{Incerteza da Análise de Teor de Água}

Serão analisadas as incertezas dos três principais métodos utilizados para análise de água em óleo a bordo de plataformas offshore: Centrifugação, Karl Fischer volumétrico e Karl Fischer coulométrico.

As amostras analisadas podem ou não conter água livre. Em geral, o fluxo de óleo fiscal não contém água livre, pois o mesmo já passou por processo de 
separação a fim de restringir a especificação do BSW deste óleo ao $1 \%$ exigido pela ANP.

Embora a definição de BSW seja o teor de água e sedimentos no óleo, nos casos estudados há apenas a presença de água no fluxo de óleo fiscal produzido. A presença de sedimentos, inclusive, pode significar problemas operacionais. Portanto, deve-se considerar como teor de água somente, em todas as instâncias do termo BSW neste capítulo.

\subsection{1}

\section{Incerteza do Método de Centrifugação}

A repetibilidade é a fonte de maior incerteza na análise de BSW (Biazon et al., 2015).

Para calcular o volume de BSW segundo a ASTM D4007 (2013) utilizando o método de centrifugação, já descrito no Capítulo 2, sem água livre, utiliza-se

$$
B S W_{i}=\left(\frac{V_{i}}{V_{p}+V_{s}} * 100\right),
$$

sendo

$V_{i}$ - volume de água e sedimentos depositado no tubo, em ml;

$V_{p}$ - volume de óleo cru, em $\mathrm{ml}$;

$V_{s}$ - volume de solvente, neste caso tolueno, em $\mathrm{ml}$;

$B S W_{i}-$ volume de BSW (teor de água) sem água livre em \% v/v.

O cálculo realizado pela equação (36) será feito para cada uma das duas amostras. Portanto, calcula-se o $B S W_{1}$ e o $B S W_{2}$, sendo o $B S W_{E}$ o resultante do método, calculado utilizando

$$
B S W_{E}=B S W_{1}+B S W_{2} .
$$

Para o cálculo da incerteza do método, considerando as equações (35), (36) e (37), as quantidades não correlacionadas e a repetibilidade (repe), tem-se

$$
\begin{aligned}
& u_{c}\left(B S W_{E}\right) \\
& =\sqrt{\left(\frac{\partial B S W_{E}}{\partial B S W_{1}} * u\left(B S W_{1}\right)\right)^{2}+\left(\frac{\partial B S W_{E}}{\partial B S W_{2}} * u\left(B S W_{2}\right)\right)^{2}+\left(\frac{\partial B S W_{E}}{\partial \text { repe }} * u(\text { repe })\right)^{2}},
\end{aligned}
$$

sendo 


$$
\begin{aligned}
& u_{c}\left(B S W_{i}\right) \\
& =\sqrt{\left(\frac{\partial B S W_{i}}{\partial V_{i}} * u\left(V_{i}\right)\right)^{2}+\left(\frac{\partial B S W_{i}}{\partial V_{p}} * u\left(V_{p}\right)\right)^{2}+\left(\frac{\partial B S W_{i}}{\partial V_{s}} * u\left(V_{s}\right)\right)^{2}} .
\end{aligned}
$$

Os coeficientes de sensibilidade, $c_{i}$, são calculados considerando

$$
\begin{gathered}
\frac{\partial B S W}{\partial V_{i}}=\frac{100}{V_{p}+V_{s}} ; \frac{\partial B S W_{i}}{\partial V_{p}}=\frac{\partial B S W_{i}}{\partial V_{S}}=-\frac{100 * V_{i}}{\left(V_{p}+V_{s}\right)^{2}} ; \\
\frac{\partial B S W_{E}}{\partial B S W_{1}}=\frac{\partial B S W_{E}}{\partial B S W_{2}}=\frac{\partial B S W_{E}}{\partial B S W_{\text {repe }}}=1 .
\end{gathered}
$$

Por fim, calculam-se os graus de liberdade efetivos utilizando

$$
V_{e f f}\left(B S W_{i}\right)=\frac{u_{c}^{4}\left(B S W_{i}\right)}{\left[\frac{\left(\frac{\partial B S W_{i}}{\partial V_{i}} * u\left(V_{i}\right)\right)^{4}}{v_{e f f}\left(V_{i}\right)}+\frac{\left(\frac{\partial B S W_{i}}{\partial V_{p}} * u\left(V_{p}\right)\right)^{4}}{v_{e f f}\left(V_{p}\right)}+\frac{\left(\frac{\partial B S W_{i}}{\partial V_{s}} * u\left(V_{s}\right)\right)^{4}}{v_{e f f}\left(V_{s}\right)}\right]^{\prime}}
$$

$$
V_{e f f}\left(B S W_{E}\right)
$$

$$
\left.=\frac{u_{c}^{4}\left(B S W_{E}\right)}{\left[\frac{\left(\frac{\partial B S W_{E}}{\partial B S W_{1}} * u\left(B S W_{1}\right)\right)^{4}}{v_{e f f}\left(B S W_{1}\right)}+\frac{\left(\frac{\partial B S W_{E}}{\partial B S W_{2}} * u\left(B S W_{2}\right)\right)^{4}}{v_{e f f}\left(B S W_{2}\right)}+\frac{\left(\frac{\partial B S W_{E}}{\partial R e p e} * u(R e p e)\right)^{4}}{v_{e f f}(\text { Repe })}\right.}\right]^{\prime}
$$

A análise do BSW utilizando a centrifugação também pode conter água livre na amostra. Neste caso, Biazon et al. (2015) indicam que se calcula o BSW utilizando

$$
B S W_{T}=100 * \frac{\left[\left(\frac{V_{E} * B S W_{E}}{100}\right)+V_{w}\right]}{V_{T}},
$$

sendo

$V_{E}$ - volume de emulsão, em ml;

$V_{w}$ - volume de água livre, em $\mathrm{ml}$;

$V_{T}-$ volume total da amostra, em $\mathrm{ml}$;

$B S W_{T}-\mathrm{BSW}$ (teor de água) da amostra, em \% v/v;

$B S W_{E}-\mathrm{BSW}$ (teor de água) da parte sem água livre, em \% v/v. 
Então, a incerteza combinada para este caso, considerando a equação (43) e a repetibilidade, pode ser calculada utilizando

$$
\begin{aligned}
& u_{c}\left(B S W_{T}\right)= \\
& \sqrt{\left(\frac{\partial B S W_{T}}{\partial B S W_{E}} * u\left(B S W_{E}\right)\right)^{2}+\left(\frac{\partial B S W_{T}}{\partial V_{W}} * u\left(V_{W}\right)\right)^{2}+\left(\frac{\partial B S W_{T}}{\partial V_{T}} * u\left(V_{T}\right)\right)^{2}+\left(\frac{\partial B S W_{T}}{\partial \text { Repe }} * u(\text { Repe })\right)^{2}},
\end{aligned}
$$

Os coeficientes de sensibilidade, $c_{i}$, serão calculados utilizando

$$
\begin{gathered}
\frac{\partial B S W_{T}}{\partial B S W_{E}}=\frac{V_{T}-V_{w}}{V_{T}} ; \frac{\partial B S W_{T}}{\partial V_{T}}=\frac{B S W_{E} * V_{T}-\left(V_{T}-V_{W}\right) * B S W_{E}-100 * V_{W}}{V_{T}^{2}} ; \\
\frac{\partial B S W_{T}}{\partial V_{W}}=\frac{-B S W_{E}+100}{V_{T}} ; \frac{\partial B S W_{T}}{\partial \operatorname{Repe}}=100 * \frac{\left[\left(\frac{V_{E}+B S W_{E}}{100}\right)+V_{w}\right]}{V_{T}} .
\end{gathered}
$$

Os graus de liberdade efetivos podem ser calculados utilizando

$$
\begin{aligned}
& V_{\text {eff }}\left(B S W_{T}\right) \\
& =\frac{u_{c}^{4}\left(B S W_{T}\right)}{\left[\frac{\left(\frac{\partial B S W_{T}}{\partial B S W_{E}} * u\left(B S W_{E}\right)\right)^{4}}{V_{e f f}\left(B S W_{E}\right)}+\frac{\left(\frac{\partial B S W_{T}}{\partial V_{W}} * u\left(V_{W}\right)\right)^{4}}{V_{e f f}\left(V_{W}\right)}+\frac{\left(\frac{\partial B S W_{T}}{\partial V_{T}} * u\left(V_{T}\right)\right)^{4}}{V_{e f f}\left(V_{T}\right)}+\frac{\left(\frac{\partial B S W_{T}}{\partial R e p e} * u(R e p e)\right)^{4}}{V_{e f f}(\text { Repe })}\right]} .
\end{aligned}
$$

Para o cálculo de cada parcela da incerteza, considerando a descrição do método de centrifugação feita no Capítulo 2 deste trabalho, serão utilizados os seguintes fatores:

A tolerância do tubo de centrifugação, conforme descrita na Tabela 1 do Capítulo 2. Considerando que esta tolerância ao redor do valor padrão siga uma distribuição retangular, calcula-se a incerteza $u_{t o l}$ utilizando

$$
u_{\text {tol }}=\frac{\text { tolerância do tubo }}{\sqrt{3}},
$$

cujo valor será calculado para cada medição realizada.

2. Aproximação das medições do volume de água em cada um dos tubos conforme descrito no Capítulo 2, e cálculo do BSW conforme a equação (2). Por fim, utiliza-se as Tabelas 2 ou 3 para obter o resultado do BSW. Esta forma de arredondamento contém uma incerteza associada que pode ser definida de acordo com a faixa 
utilizada no tubo. Considerando que esta incerteza também segue uma distribuição retangular, tem-se

$$
u_{\text {arred }}=\frac{\text { incerteza da faixa de medição do tubo }}{\sqrt{3}},
$$

considerando que a incerteza da faixa de medição é definida pela medição feita através da Figura 8.

Como exemplo, entre 0 e 0,10 $\mathrm{ml}$ há subdivisões de 0,05

ml. Considerando que a medição segue uma distribuição retangular, arredonda-se para a metade da menor divisão, neste exemplo para $0,025 \mathrm{ml}$. Esta será considerada a incerteza da faixa de medição do tubo a ser substituída na fórmula.

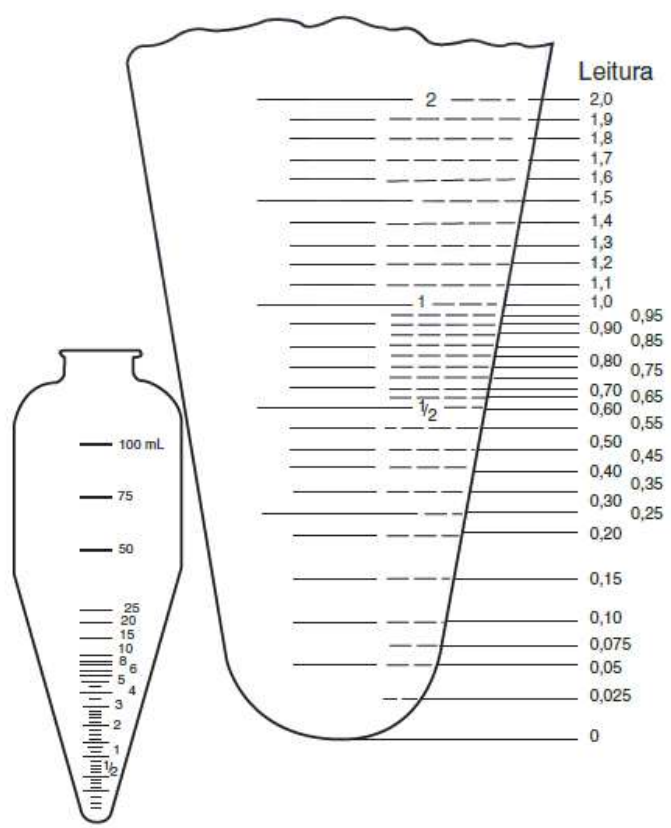

Figura 8 - Figura adaptada de ABNT NBR 14647 - Procedimento para medição do BSW em tubo cônico de $100 \mathrm{ml}$.

3. Considerando a dilatação volumétrica do vidro desprezível, calcula-se a incerteza da variação de volume do líquido dentro do tubo devido à variação de temperatura.

A medição deveria ser feita idealmente a $60{ }^{\circ} \mathrm{C}$, porém o próprio método prevê uma tolerância em torno de $\pm 3{ }^{\circ} \mathrm{C}$. Considerando o coeficiente de expansão volumétrica da água como $2,1 \times 10^{-4}{ }^{\circ} \mathrm{C}^{-1}$, do óleo e do tolueno como 
$0,001{ }^{\circ} \mathrm{C}^{-1}$ e que a variação de cada volume separado segue

$$
\Delta V=\gamma * V_{0} * \Delta T
$$

sendo

$\Delta V$ - variação de volume pela mudança de temperatura;

$\gamma$ - coeficiente de expansão térmica;

$V_{0}$ - volume inicial;

$\Delta T$ - variação de temperatura.

Considerando que a variação de volume em função da temperatura segue uma distribuição retangular, pode-se calcular a incerteza $u_{\text {temp }}$ associada a este cálculo utilizando

$$
u_{t e m p}=\frac{\Delta V}{\sqrt{3}}
$$

Considerando os fatores 1, 2 e 3 aqui descritos, calcula-se, então, a incerteza combinada $u_{c o m b}$ de cada medição de volume utilizando

$$
\mathrm{u}_{\mathrm{comb}}=\sqrt{\mathrm{u}_{\mathrm{tol}}{ }^{2}+\mathrm{u}_{\mathrm{vol}}{ }^{2}+\mathrm{u}_{\mathrm{arred}}{ }^{2}},
$$

Para o cálculo da repetibilidade utiliza-se o gráfico representado na Figura 2 até um BSW de $0,30 \%$. Acima disto até $1 \%$, a norma define que a mesma é de $0,12 \% \mathrm{v} / \mathrm{v}$.

Para obter a incerteza expandida do método, multiplica-se a incerteza encontrada pelo fator de abrangência $k$. Caso não se tenha a definição deste termo, será utilizado $k=2$.

Biazon et al. (2015) demonstram que, nos casos avaliados em seu trabalho, a repetibilidade é responsável por mais de $90 \%$ da incerteza da análise de BSW pelo método da centrifugação. Ou seja, caso seja necessário minimizar as incertezas (erros aleatórios) destes parâmetros, deve-se validar o método utilizado em cada laboratório destacando o valor mais apropriado de repetibilidade para cada operador, ao invés de utilizar os valores tabelados recomendados pelas normas, que podem estar superestimados. 


\subsection{2}

\section{Incerteza do Método Karl Fischer}

Segundo de Alencar et al. (2016), para o cálculo do teor de água pelo método Karl Fischer Volumétrico, descrito no Capítulo 2, considerando as condições padrão de $20^{\circ} \mathrm{C}$ e $101,325 \mathrm{kPa}$, utiliza-se

$$
\mathrm{KF}_{\mathrm{vol}}=\mathrm{KF}_{\mathrm{m}} * \frac{\rho_{\text {amostra }}}{\rho_{\text {água }}},
$$

sendo

$K F_{v o l}$ - teor de água da amostra em $\% \mathrm{v} / \mathrm{v}$;

$K F_{m}$ - teor de água da amostra em $\% \mathrm{~m} / \mathrm{m}$;

$\rho_{\text {amostra }}$ - massa específica da amostra em $\mathrm{g} / \mathrm{cm}^{3}$;

$\rho_{\text {óleo }}$ - massa específica do óleo em $\mathrm{g} / \mathrm{cm}^{3}$.

Considera-se a seguinte relação para o teor de água obtido experimentalmente, $\mathrm{KF}_{\text {vol }}^{\text {op }}$,

$$
\mathrm{KF}_{\mathrm{vol}}^{\mathrm{op}}=\mathrm{KF}_{\mathrm{m}} * \frac{\rho_{\text {amostra }}+\mathrm{CPL}_{\text {amostra }}+\mathrm{CTL}_{\mathrm{amostra}}}{\rho_{\text {água }}+\mathrm{CPL}_{\text {água }}+\mathrm{CTL}_{\text {água }}} \text {. }
$$

Os algoritmos para o cálculo do CPL e CTL da amostra podem ser encontrados na norma API MPMS Capítulo 11.1 (2012) e para água na API MPSM Capítulo 20.1 (2011).

Calcula-se, então, a incerteza padrão combinada, utilizando

$$
\begin{gathered}
\mathrm{u}_{\mathrm{c}}^{2}\left(\mathrm{KF}_{\mathrm{vol}}\right)=\left(\frac{\partial \mathrm{KF}_{\mathrm{vol}}}{\partial \mathrm{KF}_{\mathrm{m}}} * \mathrm{u}\left(\mathrm{KF}_{\mathrm{m}}\right)\right)^{2}+\left(\frac{\partial \mathrm{KF}_{\mathrm{vol}}}{\partial \rho_{\text {amostra }}} * \mathrm{u}\left(\rho_{\text {amostra }}\right)\right)^{2} \\
+\left(\frac{\partial \mathrm{KF}_{\mathrm{vol}}}{\partial \rho_{\text {água }}} * \mathrm{u}\left(\rho_{\text {água }}\right)\right)^{2} e \\
\mathrm{u}_{\mathrm{c}}^{2}\left(\mathrm{KF}_{\mathrm{vol}}\right)=\left(\frac{\rho_{\text {amostra }}}{\rho_{\text {água }}} * \mathrm{u}\left(\mathrm{KF}_{\mathrm{m}}\right)\right)^{2}+\left(\frac{\partial \mathrm{KF}_{\mathrm{vol}}}{\rho_{\text {água }}} * \mathrm{u}\left(\rho_{\text {amostra }}\right)\right)^{2} \\
+\left(\frac{\mathrm{KF} \mathrm{m}_{\mathrm{m}} * \rho_{\text {amostra }}}{\rho_{\text {água }}^{2}} * \mathrm{u}\left(\rho_{\text {água }}\right)\right)^{2} .
\end{gathered}
$$

Os autores descrevem também a equação (56) para o cálculo do teor de água da amostra em $\% \mathrm{~m} / \mathrm{m}$, considerando o valor para as condições padrão de $20{ }^{\circ} \mathrm{C}$ e $101,325 \mathrm{kPa}$, como 


$$
\begin{aligned}
& \mathrm{KF}_{\mathrm{m}}=\left(\mathrm{VEQ} * \mathrm{CONC}-\frac{\mathrm{B}}{1000}-\mathrm{TEMPO} * \frac{\mathrm{UMIDADE}}{60000}\right) * \frac{0,1}{\mathrm{~m}} * \text { Repe, } \\
& \text { sendo }
\end{aligned}
$$

$V E Q$ - reagente de titulação Karl Fischer necessário para titular a amostra, em ml;

CONC - concentração de reagente de titulação Karl Fischer, ou seja, a massa consumida de água em $\mathrm{mg}$ por $1 \mathrm{ml}$ de reagente;

$B$ - quantidade de água contida na garrafa menos a quantidade de água devido ao DRIFT (umidade versus tempo), ou valor branco, em $\mu \mathrm{g}$;

$T E M P O$ - tempo, em s;

$U M I D A D E$ - umidade presente na célula de reação devido ao gás de purga, em $\mu \mathrm{g} \min ^{-1}$;

$m$ - massa da amostra, em gramas;

Repe - repetibilidade, com valor numérico 1, seguindo o EURACHEM (2000).

Calcula-se, então, a incerteza padrão do método utilizando

$$
\begin{aligned}
\mathrm{u}_{\mathrm{c}}^{2}\left(\mathrm{KF}_{\mathrm{m}}\right)=\left(\frac{\partial \mathrm{KF}_{\mathrm{m}}}{\partial \mathrm{VEQ}} * \mathrm{u}(\mathrm{VEQ})\right)^{2}+\left(\frac{\partial \mathrm{KF}_{\mathrm{m}}}{\partial \mathrm{CONC}} * \mathrm{u}(\mathrm{CONC})\right)^{2}+\left(\frac{\partial \mathrm{KF}_{\mathrm{m}}}{\partial \mathrm{B}} * \mathrm{u}(\mathrm{B})\right)^{2} \\
+\left(\frac{\partial \mathrm{KF}_{\mathrm{m}}}{\partial \mathrm{TEMPO}} \mathrm{u}(\mathrm{TEMPO})\right)^{2}+\left(\frac{\partial \mathrm{KF}_{\mathrm{m}}}{\partial \mathrm{UMIDADE}} * \mathrm{u}(\text { UMIDADE })\right)^{2} \\
+\left(\frac{\partial \mathrm{KF}_{\mathrm{m}}}{\partial \mathrm{m}} * \mathrm{u}(\mathrm{m})\right)^{2}+\left(\frac{\partial \mathrm{KF}_{\mathrm{m}}}{\partial \operatorname{Repe}^{2}} * \mathrm{u}(\mathrm{Repe})\right)^{2} \mathrm{e} \\
\mathrm{u}_{\mathrm{c}}^{2}\left(\mathrm{KF}_{\mathrm{m}}\right)=\left(\frac{\text { CONC } * 0,1 * \text { Repe }}{\mathrm{m}} * \mathrm{u}(\mathrm{VEQ})\right)^{2}+\left(\frac{\text { VEQ } * 0,1 * \text { repe }}{\mathrm{m}} * \mathrm{u}(\mathrm{CONC})\right)^{2}
\end{aligned}
$$$$
+\left(-\frac{0,1 * \text { Repe }}{1000 * \mathrm{~m}} * \mathrm{u}(\mathrm{B})\right)^{2}
$$$$
+\left(-\frac{\operatorname{UMIDADE} * 0,1 * \text { Repe }}{60000 * \mathrm{~m}} * \mathrm{u}(\text { TEMPO })\right)^{2}
$$$$
+\left(-\frac{\text { TEMPO } * 0,1 * \text { Repe }}{60000 * \mathrm{~m}} * \mathrm{u}(\text { UMIDADE })\right)^{2}
$$$$
+\left(-\frac{\partial \mathrm{KF}_{\mathrm{m}}}{\mathrm{m}^{2}} * \mathrm{u}(\mathrm{m})\right)^{2}+\left(\mathrm{KF}_{\mathrm{m}} * \mathrm{u}(\text { Repe })\right)^{2},
$$

Para o cálculo da componente de incerteza dos volumes, há três contribuições: 
1 .

A incerteza da resolução da micro seringa, $u_{\text {res }}$, considerando uma distribuição retangular, portanto dividida por $\sqrt{3}$, considerando que a seringa padrão é utilizada para injetar amostras de $5 \mu 1$;

2.

A incerteza da variação de temperatura, $u_{\text {temp }}$, considerando também uma distribuição retangular, dividida por $\sqrt{3}$ e considerando o coeficiente de expansão volumétrica do solvente como $99 * 10^{-4}{ }^{\circ} \mathrm{C}^{-1}$, com a expansão do volume regida pela equação (49) e considerando uma distribuição retangular;

3. A incerteza da calibração da micro seringa, $u_{c a l}$, que está em seu certificado de calibração, em $\mu 1$, dividida pelo fator $k$ (em torno de 2 ou 3) e considerando uma distribuição retangular.

Calcula-se, então, a incerteza padrão combinada

$$
u_{\text {amostra }}=\sqrt{u_{\text {res }}^{2}+u_{\text {temp }}^{2}+u_{\text {cal }}^{2}} \text {. }
$$

Para a incerteza da massa, considerando uma distribuição normal, utilizamse as incertezas da tara e do peso bruto, conforme

$$
\mathrm{u}_{\mathrm{m}}^{2}=\left(\frac{\mathrm{U}_{\text {Tara }}}{\mathrm{k}}\right)^{2}+\left(\frac{\mathrm{U}_{\mathrm{Bruto}}}{\mathrm{k}}\right)^{2} \text {. }
$$

Calcula-se o valor B utilizando

$$
\mathrm{B}=\left(\mathrm{VEQ} * \mathrm{CONC}-\mathrm{TEMPO} * \frac{\mathrm{DRIFT}}{60000}\right) * 1000 .
$$

A incerteza combinada, considerando uma distribuição normal para este valor, pode ser calculada utilizando

$$
\begin{aligned}
\mathrm{u}_{\mathrm{c}}^{2}(\mathrm{~B})=\left(\left(\frac{\partial \mathrm{B}}{\partial \mathrm{VEQ}}\right) * \mathrm{u}(\mathrm{VEQ})\right)^{2}+\left(\left(\frac{\partial \mathrm{B}}{\partial \mathrm{CONC}}\right) * \mathrm{u}(\mathrm{CONC})\right)^{2} \\
+\left(\left(\frac{\partial \mathrm{B}}{\partial \mathrm{TEMPO}}\right) * \mathrm{u}(\text { TEMPO })\right)^{2}+\left(\left(\frac{\partial \mathrm{B}}{\partial \mathrm{DRIFT}}\right) * \mathrm{u}(\text { DRIFT })\right)^{2} \mathrm{e}
\end{aligned}
$$




$$
\begin{aligned}
\mathrm{u}_{\mathrm{c}}^{2}(\mathrm{~B}) & =(1000 * \text { CONC } * \mathrm{u}(\text { VEQ }))^{2}+(1000 * \text { VEQ } * \mathrm{u}(\text { CONC }))^{2} \\
& +\left(\left(-\frac{\text { DRIFT }}{60}\right) * \mathrm{u}(\text { TEMPO })\right)^{2} \\
& +\left(\left(-\frac{\text { TEMPO }}{60}\right) * \mathrm{u}(\text { DRIFT })\right)^{2}
\end{aligned}
$$

A concentração do reagente, $C O N C$, também considerando uma distribuição normal, pode ser calculada utilizando

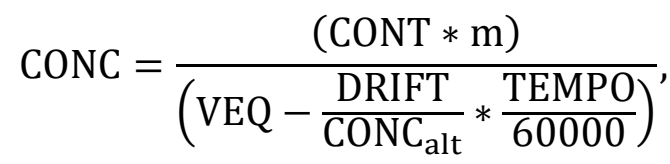

sendo:

CONT - quantidade de água purificada utilizada;

CONC $C_{a l t}$ - concentração final de reagente Karl Fischer.

Então, pode-se calcular a incerteza padrão combinada utilizando

$$
\begin{aligned}
\mathrm{u}_{\mathrm{c}}^{2}(\mathrm{CONC}) & =\left(\frac{\partial \mathrm{CONC}}{\partial \mathrm{CONT}} * \mathrm{u}(\mathrm{CONT})\right)^{2}+\left(\frac{\partial \mathrm{CONC}}{\partial \mathrm{m}} * \mathrm{u}(\mathrm{m})\right)^{2}+\left(\frac{\partial \mathrm{CONC}}{\partial \mathrm{VEQ}} * \mathrm{u}(\text { VEQ })\right)^{2} \\
& +\left(\frac{\partial \mathrm{CONC}}{\partial \mathrm{DRIFT}} * \mathrm{u}(\text { DRIFT })\right)^{2}+\left(\frac{\partial \mathrm{CONC}}{\partial \mathrm{TEMPO}} * \mathrm{u}(\text { TEMPO })\right)^{2} \\
& +\left(\frac{\partial \mathrm{CONC}^{2}}{\partial \mathrm{CONC}_{\mathrm{alt}}} * \mathrm{u}\left(\mathrm{CONC}_{\mathrm{alt}}\right)\right)^{2},
\end{aligned}
$$

considerando uma incerteza combinada do tipo A e utilizando o desvio-padrão para três repetições nos cálculos.

Por fim, calcula-se a incerteza combinada do tipo A com uma soma quadrática, dada por

$$
\begin{aligned}
& \mathrm{u}_{\mathrm{c}}^{2}(\mathrm{CONC})
\end{aligned}
$$

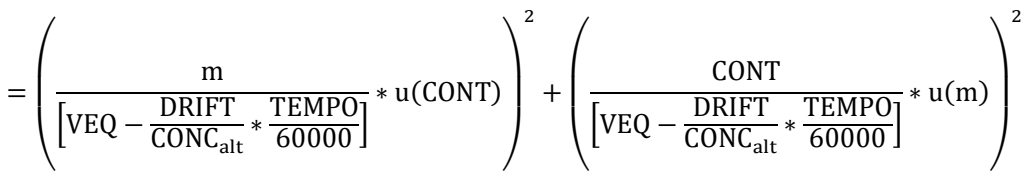

$$
\begin{aligned}
& +\left(\frac{\mathrm{CONT} * \mathrm{~m} *\left(\mathrm{CONC}_{\text {alt }} * 60000\right)^{2}}{\left(\mathrm{VEQ} * \mathrm{CONC}_{\text {alt }} * 60000-\mathrm{DRIFT} * \mathrm{TEMPO}\right)^{2}} * \mathrm{u}(\mathrm{VEQ})\right)^{2} \\
& +\left(\frac{\mathrm{CONT} * \mathrm{~m} * \mathrm{CONC}_{\text {alt }} * 60000 * \text { TEMPO }}{\left(\mathrm{VEQ} * \mathrm{CONC}_{\text {alt }} * 60000-\mathrm{DRIFT} * \mathrm{TEMPO}\right)^{2}} * \mathrm{u}(\text { DERIVA })\right)^{2}
\end{aligned}
$$

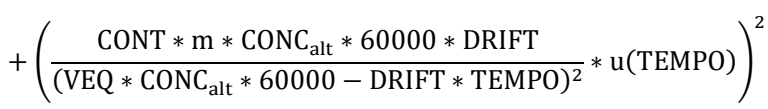

$$
\begin{aligned}
& +\left(\frac{\operatorname{CONT} * \mathrm{~m} * 60000 *\left(\mathrm{VEQ} * \mathrm{CONC}_{\text {alt }} * 60000-\mathrm{DRIFT} * \mathrm{TEMPO}\right)-\mathrm{VEQ} * 60000 *\left(\mathrm{CONT} * \mathrm{~m} * \mathrm{CONC}_{\text {alt }} * 60000\right)}{\left(\mathrm{VEQ} * \mathrm{CONC}_{\text {alt }} * 60000-\mathrm{DRIFT} * \mathrm{TEMPO}\right)^{2}}\right. \\
& \left.* \mathrm{u}\left(\mathrm{CONC}_{\text {alt }}\right)\right)^{2} \text {. }
\end{aligned}
$$


Alencar et al. (2016) utilizaram incertezas expandidas para as quantidades TEMPO ( $1 \mathrm{~s})$ e DRIFT $(0,1 \mu \mathrm{g} / \mathrm{min})$, respectivamente, ambos considerando uma distribuição retangular. Afirmam, também, que neste método de ensaio os maiores contribuintes para a incerteza são a concentração do reagente e a repetibilidade.

Segundo de Alencar et al. (2016), o método Karl Fischer é amplamente utilizado por ser rápido, exato e atender a uma ampla faixa de fração de água, apesar do custo considerado elevado.

Foi feito o levantamento dos custos para cada análise por centrifugação e por Karl Fischer Coulométrico em 2016 nas plataformas em que este estudo foi realizado e o valor é em média $50 \%$ mais caro para o segundo método, considerando que os laboratórios já estão equipados para ambas as formas de análise.

$\mathrm{Na}$ prática, ambos os métodos podem ter suas incertezas afetadas pelo operador, caso este não esteja devidamente treinado.

Ambos os métodos podem ser realizados em espaço reduzido, em um curto período e se adequam à realidade dos laboratórios de bordo. No entanto, a incerteza do método de centrifugação tende a ser maior, o que pode levar a incerteza da malha fiscal a ultrapassar o valor de $1 \%$ definido pela ANP. Para esta faixa de teor de água, as plataformas estudadas utilizam o Karl Fischer Coulométrico. 


\section{5 \\ Desenvolvimento dos experimentos}

\section{1 Montagem dos experimentos}

O planejamento de experimentos foi escolhido como ferramenta para avaliar uma possível otimização da incerteza de medição de água e analisar a influência do critério-limite de aceitação da calibração de medidores de vazão na incerteza da malha.

Um planejamento fatorial utilizando como variáveis a média da incerteza expandida da determinação de água e a média da incerteza expandida da variação do fator de calibração para cada malha avaliada foi desenhado, pois se verificou que estas são as variáveis de maior influência na incerteza das malhas, conforme pode ser visto na Tabela 7. Esta média foi obtida com os cálculos de incerteza realizados entre 2012 e 2015 para as malhas de 1 a 6 . As respostas do sistema consistem nos resultados analíticos de incerteza de cada malha de óleo para medição fiscal.

Um planejamento fatorial completo $\left(2^{k}\right)$, com um experimento no ponto central e 4 experimentos nos pontos axiais foi realizado. Os pontos axiais quando escolhidos adequadamente, permitem que o planejamento composto central seja rotacionável, isto é, o desvio-padrão da resposta prevista é considerado constante em todos os pontos que estiverem à mesma distância do centro do planejamento, sendo mais adequado para ajustes de $2^{\mathrm{a}}$ ordem. $\mathrm{O}$ número de experimentos a serem realizados será calculado por

$$
2^{k}+2 k+1 \therefore 2^{2}+2 * 2+1=9
$$

$O$ ponto de partida adotado para cada fator foi a média da influência de cada parcela da incerteza de cada malha, conforme Tabela 7. Considerou-se que os dados de todas as parcelas seguem uma distribuição de probabilidade Normal, conforme demonstrado no Anexo A pelo teste de Kolmogorov-Smirnov ao nível de significância de $1 \%$.

O modelo utilizado é o quadrático com a configuração conforme equação (21), sendo $x_{1}$ o nível da incerteza expandida de medição da análise de água e $x_{2} \mathrm{o}$ 
nível da incerteza expandida da variação do fator de calibração. Os coeficientes $b$ foram calculados utilizando o cálculo matricial da equação (25).

Utilizando a superfície de resposta, localiza-se o ponto crítico.

Para decodificar os níveis, ou seja, calcular as variáveis codificadas, utilizouse a equação (27).

Utilizaram-se os valores médios da incerteza padrão do teor de água e do fator do medidor para a composição do experimento, variando estes dois e calculando as mudanças das incertezas da malha em $50 \%$ cada. As demais parcelas da incerteza, por terem uma influência pequena na composição da incerteza da malha, foram mantidas constantes.

Pode-se ver nas Tabelas 8 e 9 os valores calculados para a Malha 1 .

Tabela 8 - Variação dos valores dos componentes da incerteza da Malha 1 em 50 \%, em m³.

\begin{tabular}{|c|c|c|c|}
\hline & $\mathbf{- 1}$ & $\mathbf{0}$ & $+\mathbf{1}$ \\
\hline MF & 0,003035 & 0,006069 & 0,009104 \\
\hline Água & 0,004235 & 0,00847 & 0,012705 \\
\hline
\end{tabular}

Tabela 9 - Experimento incerteza de água $\mathrm{x}$ incerteza da variação do MF para a Malha 1.

\begin{tabular}{|c|c|c|c|c|c|}
\hline Experimento & $\begin{array}{c}\text { Água } \\
(\mathbf{\%} \text { v/v) }\end{array}$ & $\begin{array}{c}\mathbf{M F} \\
\mathbf{( m}^{\mathbf{3}} \mathbf{)}\end{array}$ & $\mathbf{x}_{\mathbf{l}}$ & $\mathbf{x}_{\mathbf{2}}$ & $\begin{array}{c}\text { y (incerteza da } \\
\text { malha })\end{array}$ \\
\hline $\mathbf{1}$ & 0,004235 & 0,003035 & -1 & -1 & $0,09 \%$ \\
\hline $\mathbf{2}$ & 0,012705 & 0,003035 & +1 & -1 & $0,20 \%$ \\
\hline $\mathbf{3}$ & 0,004235 & 0,009104 & -1 & +1 & $0,15 \%$ \\
\hline $\mathbf{4}$ & 0,012705 & 0,009104 & +1 & +1 & $0,23 \%$ \\
\hline $\mathbf{5}$ & 0,008470 & 0,006069 & 0 & 0 & $0,16 \%$ \\
\hline $\mathbf{6}$ & 0,002482 & 0,006069 & $-1,414$ & 0 & $0,10 \%$ \\
\hline $\mathbf{7}$ & 0,014458 & 0,006069 & $+1,414$ & 0 & $0,24 \%$ \\
\hline $\mathbf{8}$ & 0,008470 & 0,001778 & 0 & $-1,414$ & $0,14 \%$ \\
\hline $\mathbf{9}$ & 0,008470 & 0,010360 & 0 & $+1,414$ & $0,20 \%$ \\
\hline
\end{tabular}

Procedendo desta forma para cada malha, encontraram-se os coeficientes $b_{i}$ referentes à equação (21) utilizando o software Statistica, conforme demonstra a Tabela 10. 
Tabela 10 - Coeficientes do modelo quadrático para cada malha

\begin{tabular}{|c|c|c|c|c|c|c|}
\hline & Malha 1 & Malha 2 & Malha 3 & Malha 4 & Malha 5 & Malha 6 \\
\hline $\mathrm{b}_{0}$ & 0,00024 & 0,00068 & 0,00020 & 0,00002 & 0,00029 & 0,00031 \\
\hline $\mathrm{b}_{1}$ & 0,10862 & 0,05223 & 0,09484 & 0,11121 & 0,07725 & 0,08202 \\
\hline $\mathrm{b}_{2}$ & 0,06379 & 0,03864 & 0,09196 & 0,11560 & 0,07370 & 0,05213 \\
\hline $\mathrm{b}_{3}$ & $-5,83620$ & 0,00000 & $-6,14070$ & $-6,97360$ & $-5,10160$ & $-7,70376$ \\
\hline $\mathrm{b}_{4}$ & 2,43894 & 2,13149 & 3,26894 & 2,80500 & 4,01003 & 4,94341 \\
\hline $\mathrm{b}_{5}$ & 4,75054 & 2,98606 & 3,30369 & 2,12492 & 3,64982 & 6,75343 \\
\hline
\end{tabular}

Fazendo a análise dos termos por meio do método ANOVA, conforme detalhado na Tabela 11, e avaliando o gráfico de Pareto das estimativas dos efeitos padronizados, na Figura 9, observa-se que tanto os termos lineares quanto o termo de interação das variáveis são estatisticamente significativos. Os termos quadráticos de ambas as variáveis são marginalmente significativos, porém optou-se por mantêlos.

Tabela 11 - Tabela ANOVA calculada para os termos da regressão da Malha 1.

\begin{tabular}{|c|c|c|c|c|c|}
\hline \multicolumn{7}{|c|}{ ANOVA - Malha 1 } \\
\hline & $\begin{array}{c}\text { Soma dos } \\
\text { Quadrados }\end{array}$ & $\begin{array}{c}\text { Graus de } \\
\text { Liberdade }\end{array}$ & $\begin{array}{c}\text { Quadrados } \\
\text { Médios }\end{array}$ & Estatística F & p-valor \\
\hline (1)Água(L) & $1,882 \mathrm{E}-06$ & 1 & $1,882 \mathrm{E}-06$ & 2370,811 & 0,000019 \\
\hline Água (Q) & $5,566 \mathrm{E}-09$ & 1 & $5,566 \mathrm{E}-09$ & 7,013 & 0,077107 \\
\hline (2)MF (L) & $3,822 \mathrm{E}-07$ & 1 & $3,822 \mathrm{E}-07$ & 481,545 & 0,000207 \\
\hline MF (Q) & $5,567 \mathrm{E}-09$ & 1 & $5,567 \mathrm{E}-09$ & 7,014 & 0,077103 \\
\hline 1L by 2L & $2,250 \mathrm{E}-08$ & 1 & $2,250 \mathrm{E}-08$ & 28,350 & 0,012944 \\
\hline Erro & $2,381 \mathrm{E}-09$ & 3 & $7,937 \mathrm{E}-10$ & & \\
\hline Total SS & $2,296 \mathrm{E}-06$ & 8 & & & \\
\hline
\end{tabular}

Sendo:

(1)Água(L) - termo linear da variável incerteza da água - termo $b_{1}$ na Tabela 10

Água (Q) - termo quadrático da variável incerteza da água - termo $b_{4}$;

(2)MF (L) - termo linear da variável fator do medidor - termo $b_{2}$;

MF (Q) - termo quadrático da variável fator do medidor - termo $b_{5}$;

$1 \mathrm{~L}$ by $2 \mathrm{~L}$ - termo de interação entre as variáveis - termo $b_{3}$;

Total SS - total da soma dos quadrados. 


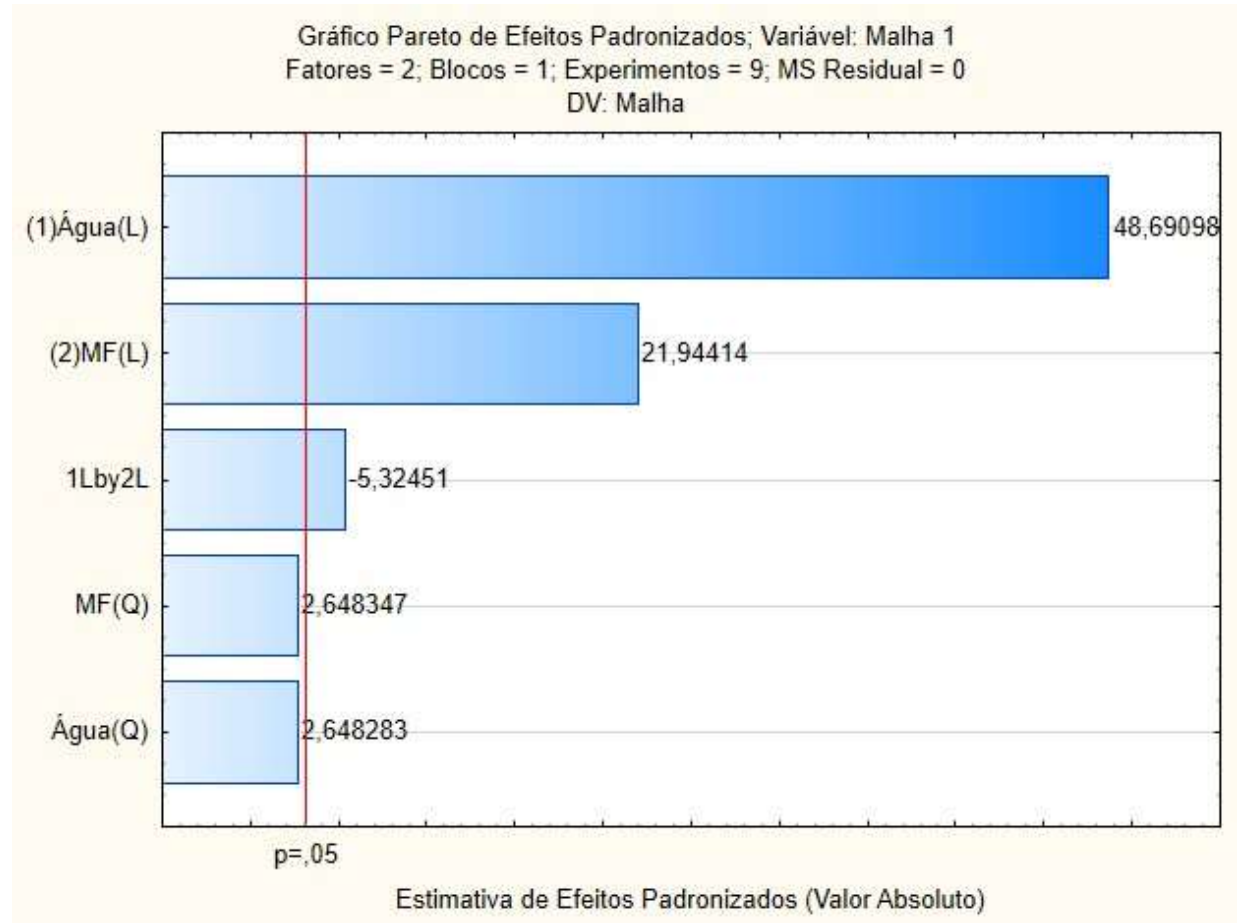

Figura 9 - Gráfico de Pareto das estimativas de efeito padronizado - Malha 1.

Nas Figuras 10 e 11, pode-se observar o comportamento dos resíduos deste planejamento de experimentos para a Malha 1. Os resíduos estão distribuídos aleatoriamente em torno do eixo $x$, o que é um indício de que o modelo proposto pela regressão está adequado. Na Figura 11, o valor do $\mathrm{R}^{2}$ calculado é 0,999, também sendo um bom indício de adequação do modelo.

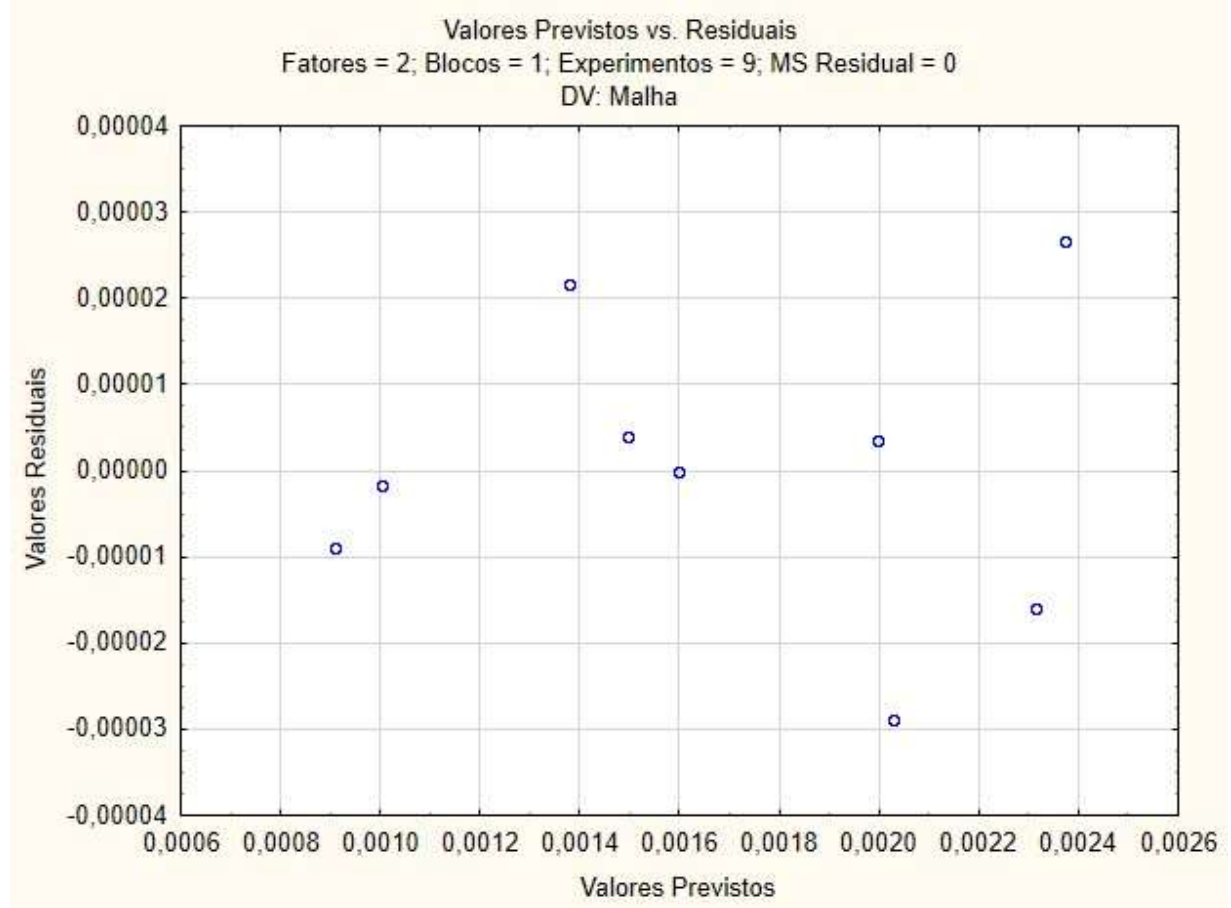

Figura 10 - Gráfico de Resíduos x Valores Previstos para Malha 1. 


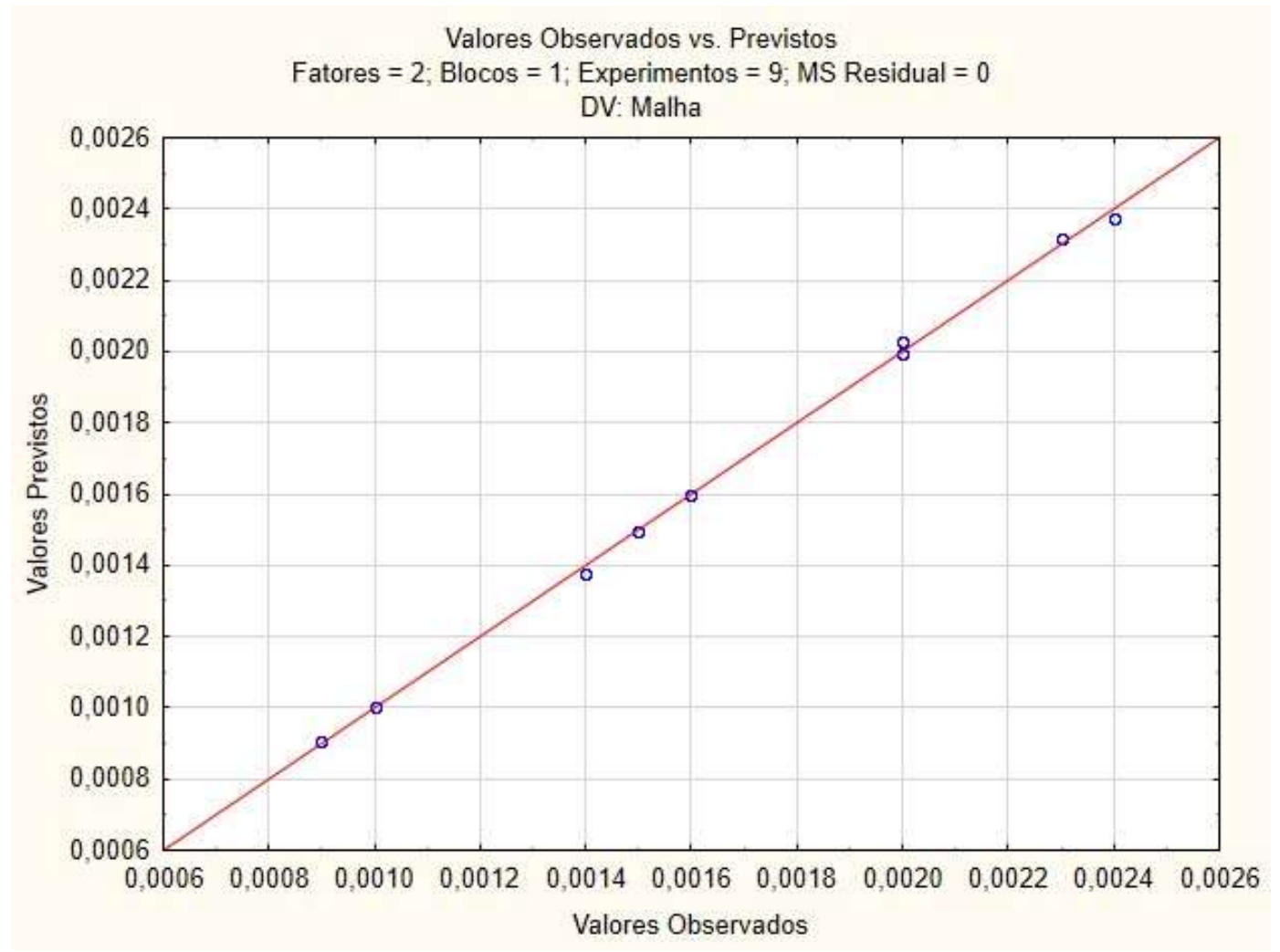

Figura 11 - Gráfico Valores Observados x Valores Previstos para Malha 1.

\section{2}

\section{Resultados dos experimentos e superfície de resposta}

A próxima etapa foi a construção do experimento para cada uma das malhas. Detalhou-se, então, o experimento para a Malha 1:

A incerteza média do teor de água para Malha 1 utilizada foi $0,008470 \% \mathrm{v} / \mathrm{v}$ e a incerteza do MF para Malha 1 foi de $0,006069 \mathrm{~m}^{3}$. Foi calculada uma variação de $\pm 100 \%$ para cada variável, de $20 \%$ em $20 \%$.

Considerando as Tabelas 8 e 9, têm-se os resultados expressos na Tabela 12. Tabela 12 - Resultados do Experimento para Malha 1 com variação de $20 \%$.

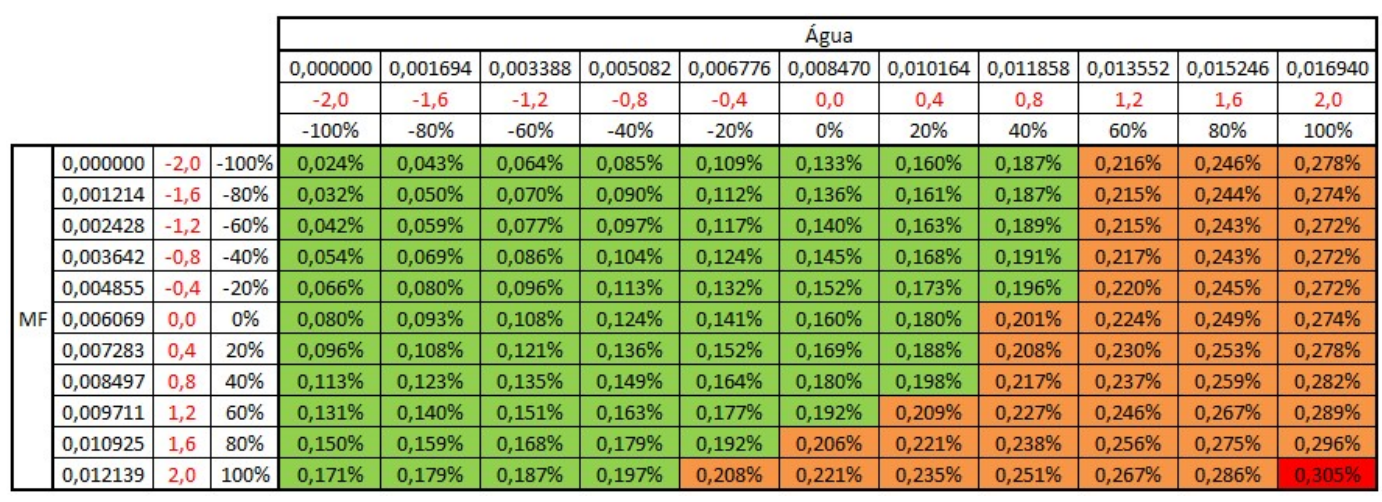


Pode-se observar na cor preta a variação da variável real. Logo abaixo, em vermelho, a variável codificada. Por último, a porcentagem de variação da variável real. Para ser possível perceber a curvatura da superfície de resposta, muda-se o degrau de variação para $35 \%$, conforme Tabela 13 .

Tabela 13 - Resultados do Experimento para Malha 1 com variação de $35 \%$.

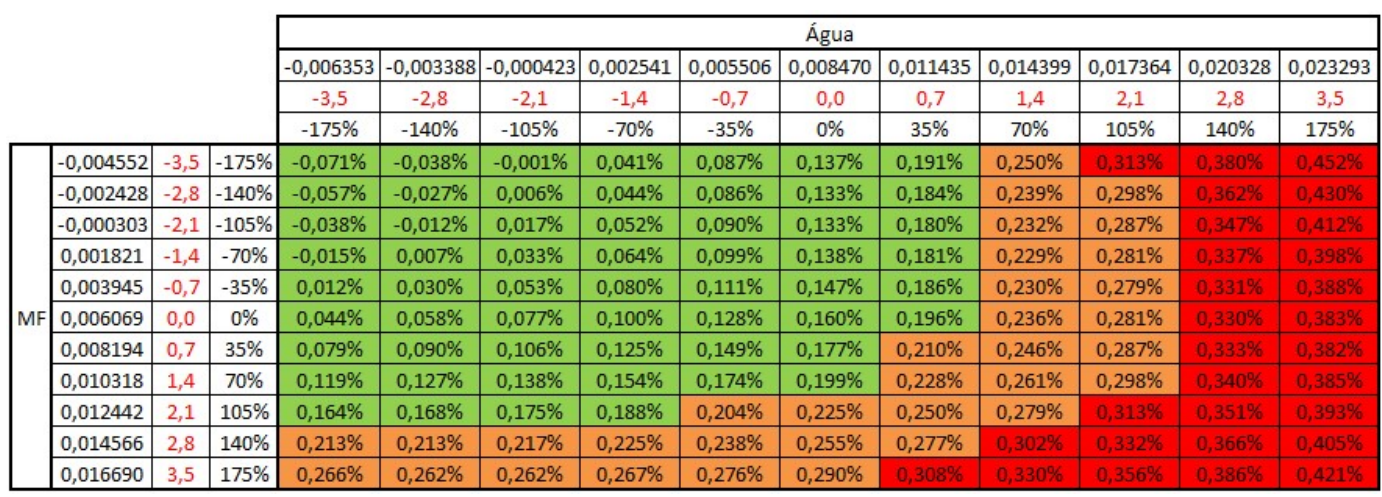

O software Statistica calcula os dados da Tabela 14, com os extremos dos valores observados e o valor crítico, o qual obteria a menor incerteza da malha analisada.

Tabela 14 - Valores máximos e mínimos do experimento, para Malha 1.

\begin{tabular}{|c|c|c|c|}
\hline & Valor Mínimo Observado & Valor Crítico & Valor Máximo Observado \\
\hline Água & 0,002481 & $-0,114327$ & 0,014459 \\
\hline MF & 0,001778 & $-0,076942$ & 0,010360 \\
\hline
\end{tabular}

Considerando que as incertezas para cada elemento calculado devem ser positivas, e os valores do ponto crítico encontrados são negativos, não há o que se analisar em relação a estes. Observando a Tabela 12, pode-se observar que para um aumento da incerteza do teor de água de $100 \%$ (2,0 na variável codificada) e 100 $\%$ de MF (2,0 na variável codificada) a incerteza da malha ultrapassa o valor definido pela ANP de $0,3 \%$.

Nas Figuras 12 e 13 são apresentadas a superfície de resposta e a visualização das curvas de nível no plano MF-água. Ao analisar esta superfície, pode-se observar que há uma maior influência na incerteza da malha na mudança da variável água em relação à variável MF. Ao se aproximar de 100 \% de variação de ambas, a incerteza da malha ultrapassa os $0,3 \%$ permitidos pelo RTM.

De forma simplificada, os dados serão apresentados para as demais malhas. 
Superficie de Resposta: Variável: Malha 1

Fatores $=2 ;$ Blocos $=1 ;$ Experimentos $=9 ;$ MS Residual $=0$ DV: Malha

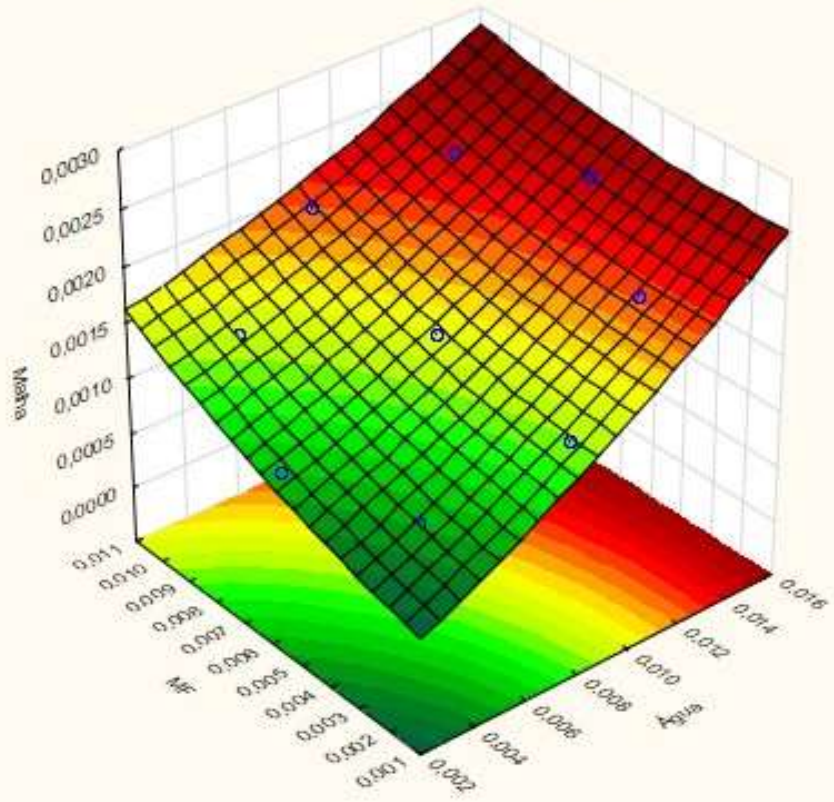

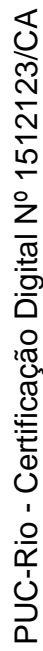

Figura 12 - Superfície de Resposta para a Malha 1.

Curva de Nível; Variável: Malha 1

Fatores $=2 ;$ Blocos $=1 ;$ Experimentos $=9 ; \mathrm{MS}$ Residual $=0$

DV: Malha

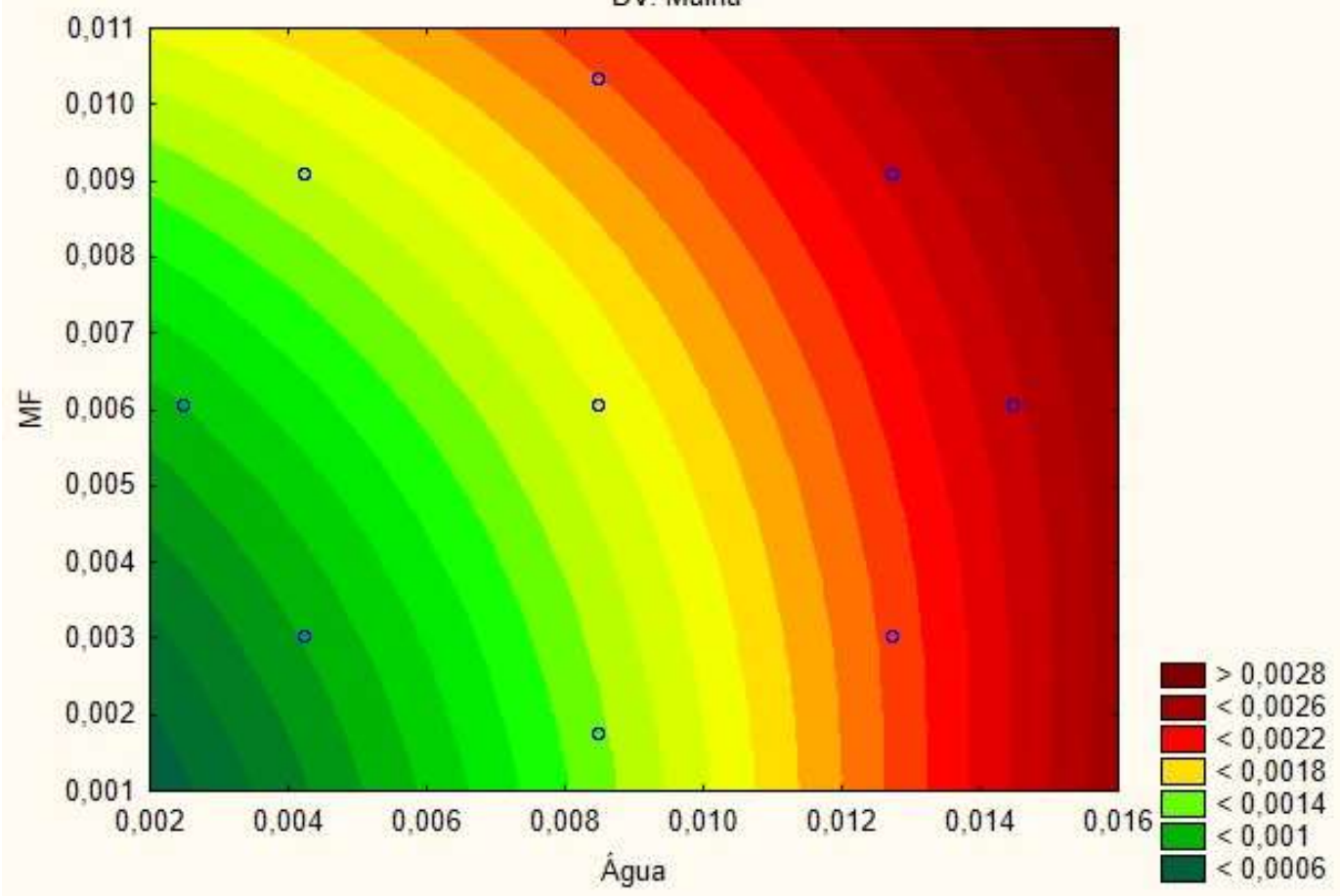

Figura 13 - Curva de Nível - Malha 1. 
- Malha 2:

Tabela 15 - Tabela ANOVA calculada para os termos da regressão da Malha 2.

\begin{tabular}{|c|c|c|c|c|c|}
\hline \multicolumn{7}{|c|}{ ANOVA - Malha 2 } \\
\hline & $\begin{array}{c}\text { Soma dos } \\
\text { Quadrados }\end{array}$ & $\begin{array}{c}\text { Graus de } \\
\text { Liberdade }\end{array}$ & $\begin{array}{c}\text { Quadrados } \\
\text { Médios }\end{array}$ & $\begin{array}{c}\text { Estatística } \\
\text { F }\end{array}$ & p-valor \\
\hline (1)Água (L) & 0,000002 & 1 & 0,000002 & 141,192 & $2,149 \mathrm{E}-05$ \\
\hline Água (Q) & $2,500 \mathrm{E}-08$ & 1 & $2,500 \mathrm{E}-08$ & 1,554 & 0,258999 \\
\hline (2)MF (L) & $1,457 \mathrm{E}-06$ & 1 & $1,457 \mathrm{E}-06$ & 90,576 & $7,675 \mathrm{E}-05$ \\
\hline MF (Q) & $2,500 \mathrm{E}-08$ & 1 & $2,500 \mathrm{E}-08$ & 1,554 & 0,258999 \\
\hline 1L by 2L & $1,000 \mathrm{E}-08$ & 1 & $1,000 \mathrm{E}-08$ & 0,622 & 0,460454 \\
\hline Erro & $9,652 \mathrm{E}-08$ & 3 & $1,609 \mathrm{E}-08$ & & \\
\hline Total SS & 0,000004 & 8 & & & \\
\hline
\end{tabular}

Gráfico Pareto de Efeitos Padronizados; Variável: Malha

Fatores $=2$, Blocos $=1$, Experimentos $=9 ;$ MS Residual $=0$ DV: Malha

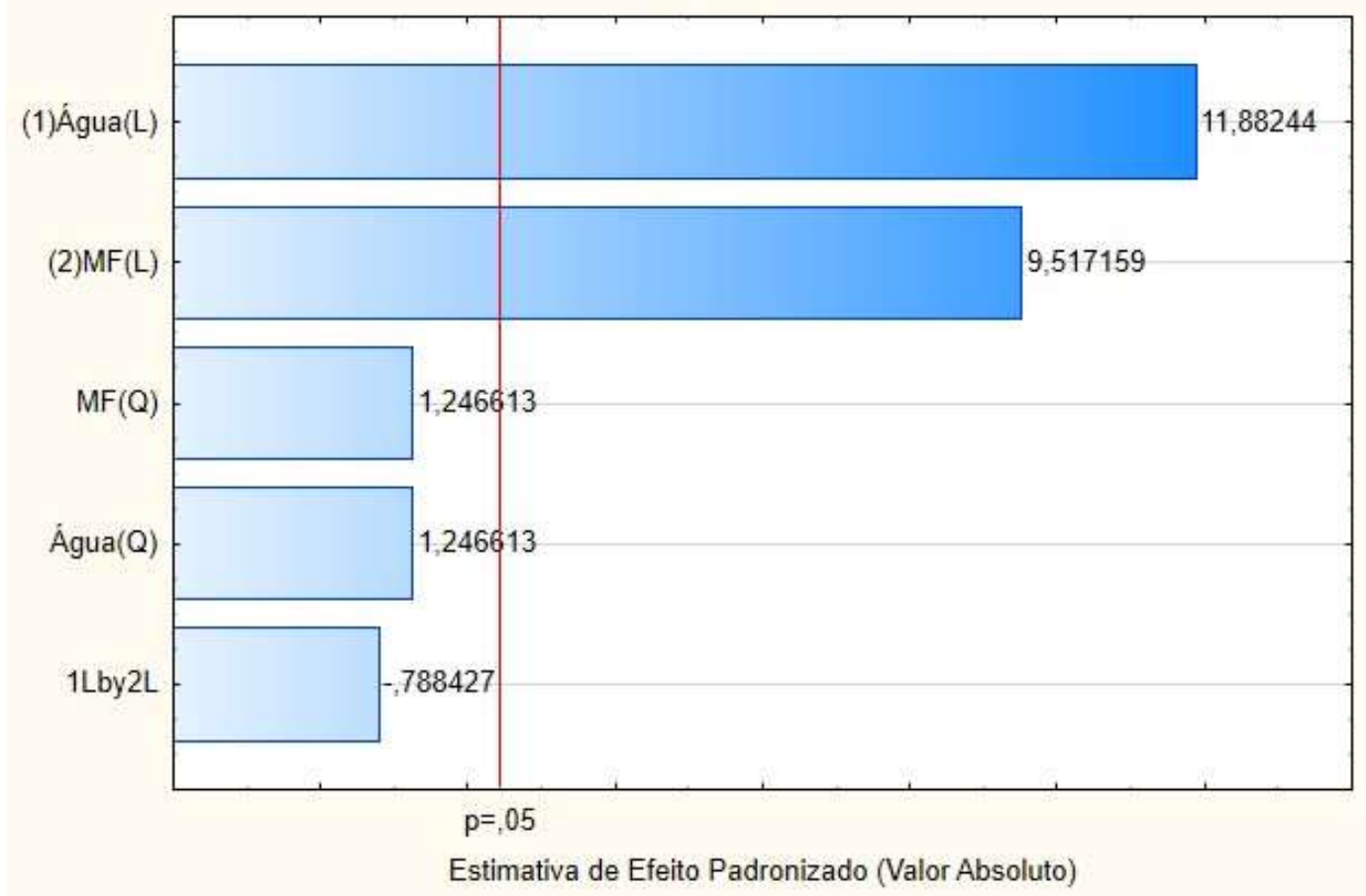

Figura 14 - Gráfico de Pareto das estimativas de efeito padronizado - Malha 2.

No gráfico de Pareto da Figura 14 pode-se observar que os termos lineares para incerteza de água e do MF são estatisticamente relevantes e que o termo que multiplica ambas é irrelevante estatisticamente. Os termos quadráticos para ambos são marginalmente significativos, mas escolheu-se por mantê-los, como pode ser visto na coluna Malha 2 da Tabela 10.

Observa-se na Figura 15 que os resíduos estão aleatoriamente distribuídos em torno do eixo $x$, sinal de uma boa adequação da regressão do modelo proposto. 


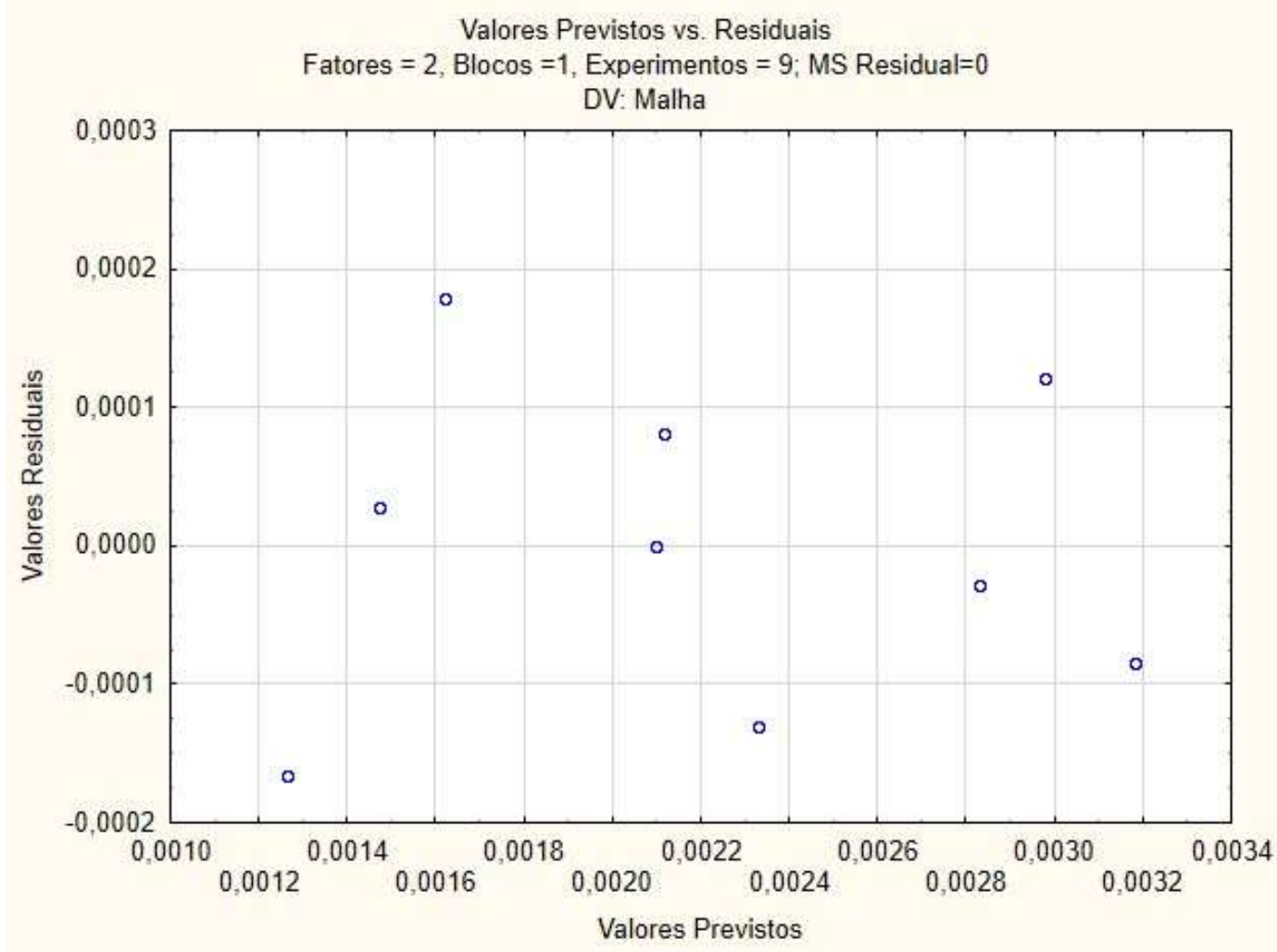

Figura 15 - Gráfico de Resíduos x Valores Previstos para Malha 2.

Tabela 16 - Resultados do Experimento para Malha 2

\begin{tabular}{|c|c|c|c|c|c|c|c|c|c|c|c|c|c|c|}
\hline & & & & & & & & & Água & & & & & \\
\hline & & & & 0,00000 & 0,00217 & 0,00433 & 0,00650 & 0,00866 & 0,01083 & 0,01300 & 0,01516 & 0,01733 & 0,01949 & 0,02166 \\
\hline & & & & $-2,0$ & $-1,6$ & $-1,2$ & $-0,8$ & $-0,4$ & 0,0 & 0,4 & 0,8 & 1,2 & 1,6 & 2,0 \\
\hline & & & & $-100 \%$ & $-80 \%$ & $-60 \%$ & $-40 \%$ & $-20 \%$ & $0 \%$ & $20 \%$ & $40 \%$ & $60 \%$ & $80 \%$ & $100 \%$ \\
\hline & 0,00000 & $-2,0$ & $-100 \%$ & $0,068 \%$ & $0,080 \%$ & $0,095 \%$ & $0,111 \%$ & $0,129 \%$ & $0,150 \%$ & $0,172 \%$ & $0,196 \%$ & $0,223 \%$ & $0,251 \%$ & $0,281 \%$ \\
\hline & 0,00183 & $-1,6$ & $-80 \%$ & $0,076 \%$ & $0,088 \%$ & $0,103 \%$ & $0,119 \%$ & $0,137 \%$ & $0,158 \%$ & $0,180 \%$ & $0,204 \%$ & $0,231 \%$ & $0,259 \%$ & $0,289 \%$ \\
\hline & 0,00366 & $-1,2$ & $-60 \%$ & $0,086 \%$ & $0,099 \%$ & $0,113 \%$ & $0,129 \%$ & $0,147 \%$ & $0,168 \%$ & $0,190 \%$ & $0,214 \%$ & $0,241 \%$ & $0,269 \%$ & $0,299 \%$ \\
\hline & 0,00549 & $-0,8$ & $-40 \%$ & $0,098 \%$ & $0,111 \%$ & $0,125 \%$ & $0,141 \%$ & $0,160 \%$ & $0,180 \%$ & $0,202 \%$ & $0,227 \%$ & $0,253 \%$ & $0,281 \%$ & $0,311 \%$ \\
\hline & 0,00732 & $-0,4$ & $-20 \%$ & $0,112 \%$ & $0,125 \%$ & $0,139 \%$ & $0,155 \%$ & $0,174 \%$ & $0,194 \%$ & $0,216 \%$ & $0,241 \%$ & $0,267 \%$ & $0,295 \%$ & $0,326 \%$ \\
\hline MF & 0,00915 & 0,0 & $0 \%$ & $0,128 \%$ & $0,141 \%$ & $0,155 \%$ & $0,171 \%$ & $0,190 \%$ & $0,210 \%$ & $0,232 \%$ & $0,257 \%$ & $0,283 \%$ & $0,311 \%$ & $0,342 \%$ \\
\hline & 0,01098 & 0,4 & $20 \%$ & $0,147 \%$ & $0,159 \%$ & $0,173 \%$ & $0,189 \%$ & $0,208 \%$ & $0,228 \%$ & $0,250 \%$ & $0,275 \%$ & $0,301 \%$ & $0,329 \%$ & $0,360 \%$ \\
\hline & 0,01281 & 0,8 & $40 \%$ & $0,167 \%$ & $0,179 \%$ & $0,193 \%$ & $0,210 \%$ & $0,228 \%$ & $0,248 \%$ & $0,270 \%$ & $0,295 \%$ & $0,321 \%$ & $0,349 \%$ & $0,380 \%$ \\
\hline & 0,01464 & 1,2 & $60 \%$ & $0,189 \%$ & $0,201 \%$ & $0,215 \%$ & $0,232 \%$ & $0,250 \%$ & $0,270 \%$ & $0,293 \%$ & $0,317 \%$ & $0,343 \%$ & $0,371 \%$ & $0,402 \%$ \\
\hline & 0,01647 & 1,6 & $80 \%$ & $0,213 \%$ & $0,225 \%$ & $0,239 \%$ & $0,256 \%$ & $0,274 \%$ & $0,294 \%$ & $0,317 \%$ & $0,341 \%$ & $0,367 \%$ & $0,396 \%$ & $0,426 \%$ \\
\hline & 0,01830 & 2,0 & $100 \%$ & $0,239 \%$ & $0,251 \%$ & $0,265 \%$ & $0,282 \%$ & $0,300 \%$ & $0,320 \%$ & $0,343 \%$ & $0,367 \%$ & $0,393 \%$ & $0,422 \%$ & $0,452 \%$ \\
\hline
\end{tabular}

A incerteza média do teor de água para Malha 2 utilizada foi 0,01083 \% v/v e a incerteza do MF para Malha 2 foi de $0,00915 \mathrm{~m}^{3}$.

Como na Malha 1, o ponto crítico calculado é negativo, portanto não faz sentido analisá-lo. Avaliando a Tabela 16, há uma área no canto inferior direito em que se ultrapassa o valor máximo da incerteza da malha exigida pelo RTM. Com variações muito baixas das variáveis, por exemplo $40 \%$ para ambas, já se está no limiar da faixa permitida. O mesmo pode ser observado na superfície de resposta e curva de nível das Figuras 16 e 17. 
Superficie de Resposta: Variável: Malha 2

Fatores $=2$, Blocos $=1$, Experimentos $=9 ;$ MS Residual $=0$ DV: Malha

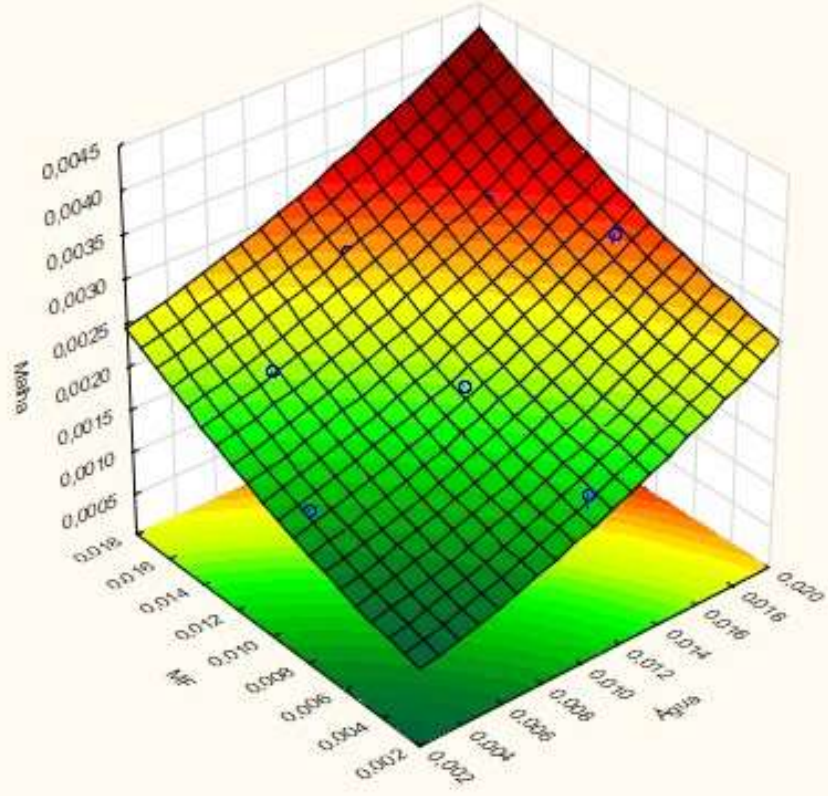

$>0,004$

$<0,0039$

$<0,0034$

$<0,0029$

$<0,0024$

$<0,0019$

$<0,0014$

$<0,0009$

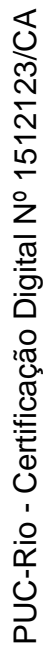

Figura 16 - Superfície de Resposta para a Malha 2.

Curvas de Nivel; Variable: Malha 2

Fatores $=2$, Blocos $=1$, Experimentos $=9 ;$ MS Residual $=0$

DV: Malha

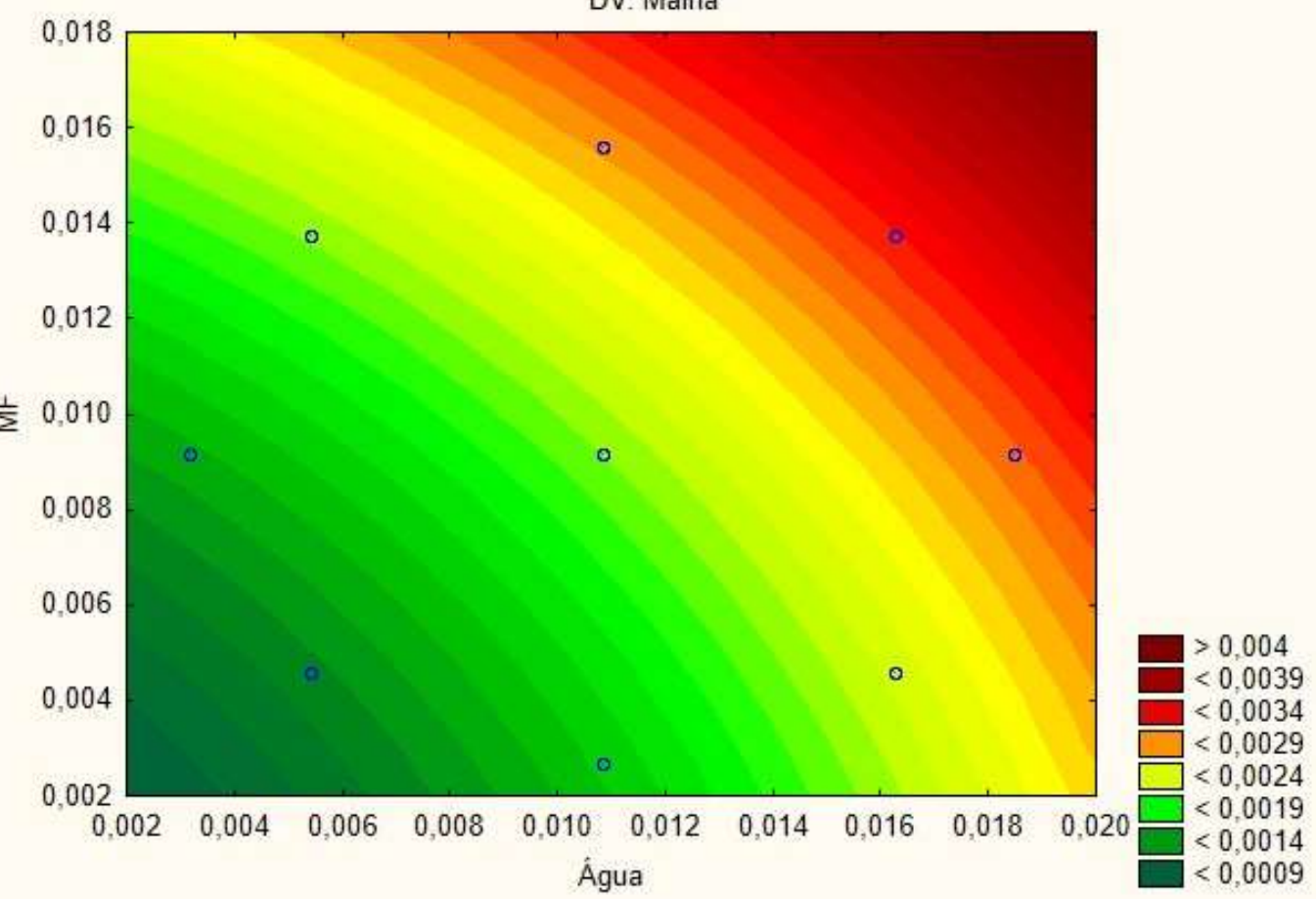

Figura 17 - Curva de Nível - Malha 2. 
- Malha 3:

Tabela 17 - Tabela ANOVA calculada para os termos da regressão da Malha 3.

\begin{tabular}{|l|c|c|c|c|c|}
\hline & $\begin{array}{c}\text { Soma dos } \\
\text { Quadrados }\end{array}$ & $\begin{array}{c}\text { Graus de } \\
\text { Liberdade }\end{array}$ & $\begin{array}{c}\text { Quadrados } \\
\text { Médios }\end{array}$ & $\begin{array}{c}\text { Estatística } \\
\text { F }\end{array}$ & p-valor \\
\hline (1)Água (L) & 0,000003 & 1 & 0,000003 & 2113,781 & $2,265 \mathrm{E}-05$ \\
\hline Água (Q) & $2,556 \mathrm{E}-08$ & 1 & $2,556 \mathrm{E}-08$ & 19,883 & 0,020999 \\
\hline (2)MF (L) & $1,245 \mathrm{E}-06$ & 1 & $1,245 \mathrm{E}-06$ & 968,229 & $7,293 \mathrm{E}-05$ \\
\hline MF (Q) & $1,375 \mathrm{E}-08$ & 1 & $1,375 \mathrm{E}-08$ & 10,692 & 0,046781 \\
\hline 1L by 2L & $9,000 \mathrm{E}-08$ & 1 & $9,000 \mathrm{E}-08$ & 70,002 & 0,003580 \\
\hline Erro & $3,857 \mathrm{E}-09$ & 3 & $1,286 \mathrm{E}-09$ & & \\
\hline Total SS & 0,000004 & 8 & & & \\
\hline
\end{tabular}

Gráfico Pareto de Efeitos Padronizados; Variável: Malha 3

Fatores $=2$, Blocos $=1$, Experimentos $=9 ;$ MS Residual $=0$

DV: Malha

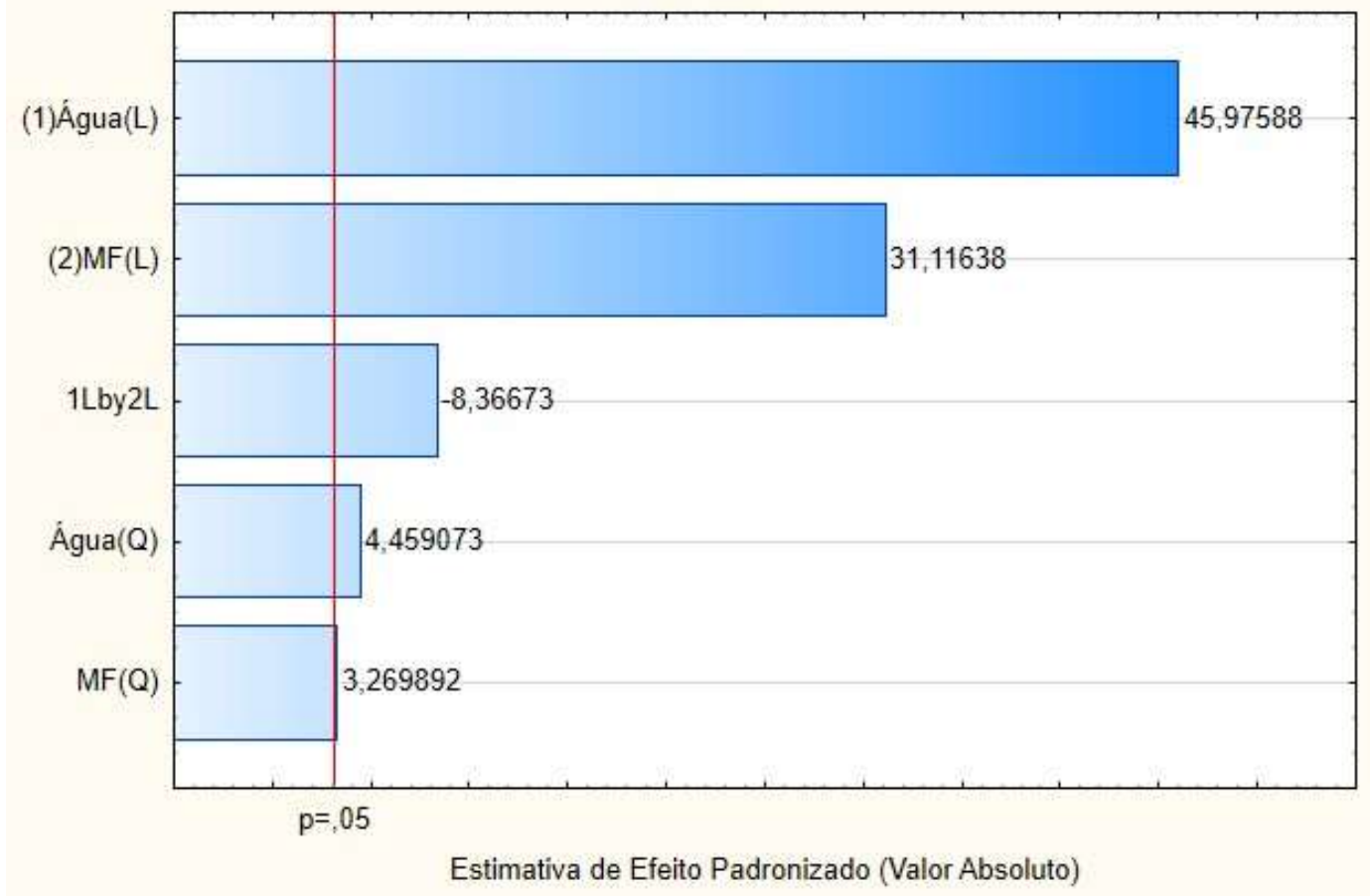

Figura 18 - Gráfico de Pareto das estimativas de efeito padronizado - Malha 3.

Neste caso, pode-se observar no Pareto da Figura 18 que todos os termos são estatisticamente relevantes. Na Figura 19 pode-se ver que o comportamento dos resíduos novamente indica uma boa adequação do modelo. Por fim, gerou-se a Tabela 18 com os resultados do experimento. 


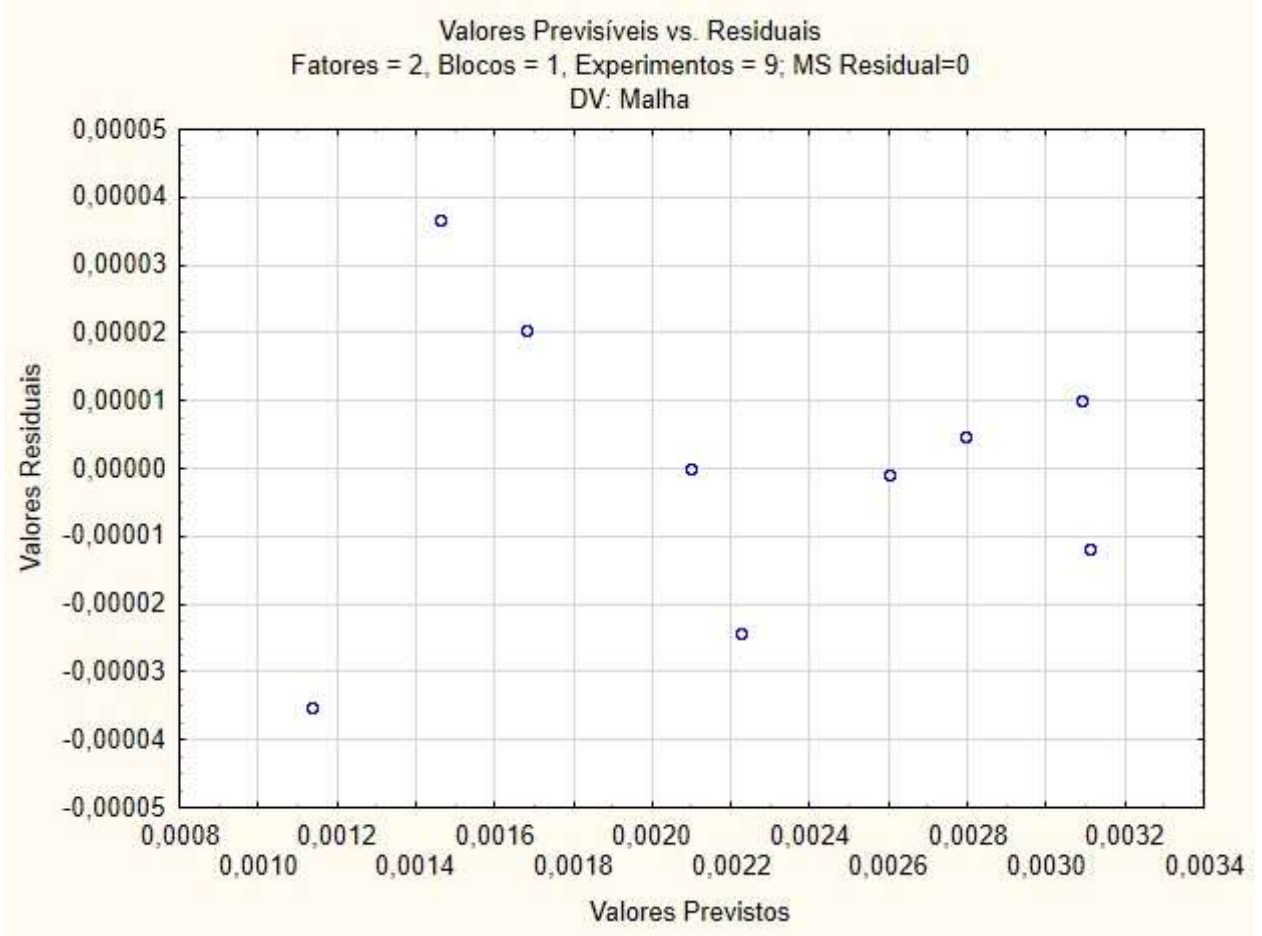

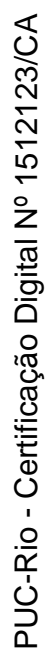

Figura 19 - Gráfico de Resíduos x Valores Previstos para Malha 3.

Tabela 18 - Resultados do Experimento para Malha 3.



A incerteza média do teor de água para Malha 3 utilizada foi $0,010710 \% \mathrm{v} / \mathrm{v}$ e a incerteza do MF para Malha 3 foi de $0,009123 \mathrm{~m}^{3}$.

Tem-se uma grande área na direita da Tabela 18 em que se ultrapassou o valor permitido para a incerteza da malha. Com variações maiores do que $80 \%$ da incerteza do teor de água todos os casos analisados ultrapassaram este valor. Com variações de $60 \%$ do teor de água e $20 \%$ de MF já se atinge o valor limite.

Para as variações de MF, os aumentos da incerteza da malha são mais suaves. Com aumento de $80 \%$, sem variação da água, por exemplo, chega-se perto do valor limite. Estas observações também podem ser vistas nas Figuras 20 e 21. 
Superficie de Resposta: Variável: Malha 3

Fatores $=2$, Blocos $=1$, Experimentos $=9 ;$ MS Residual $=0$ DV: Malha
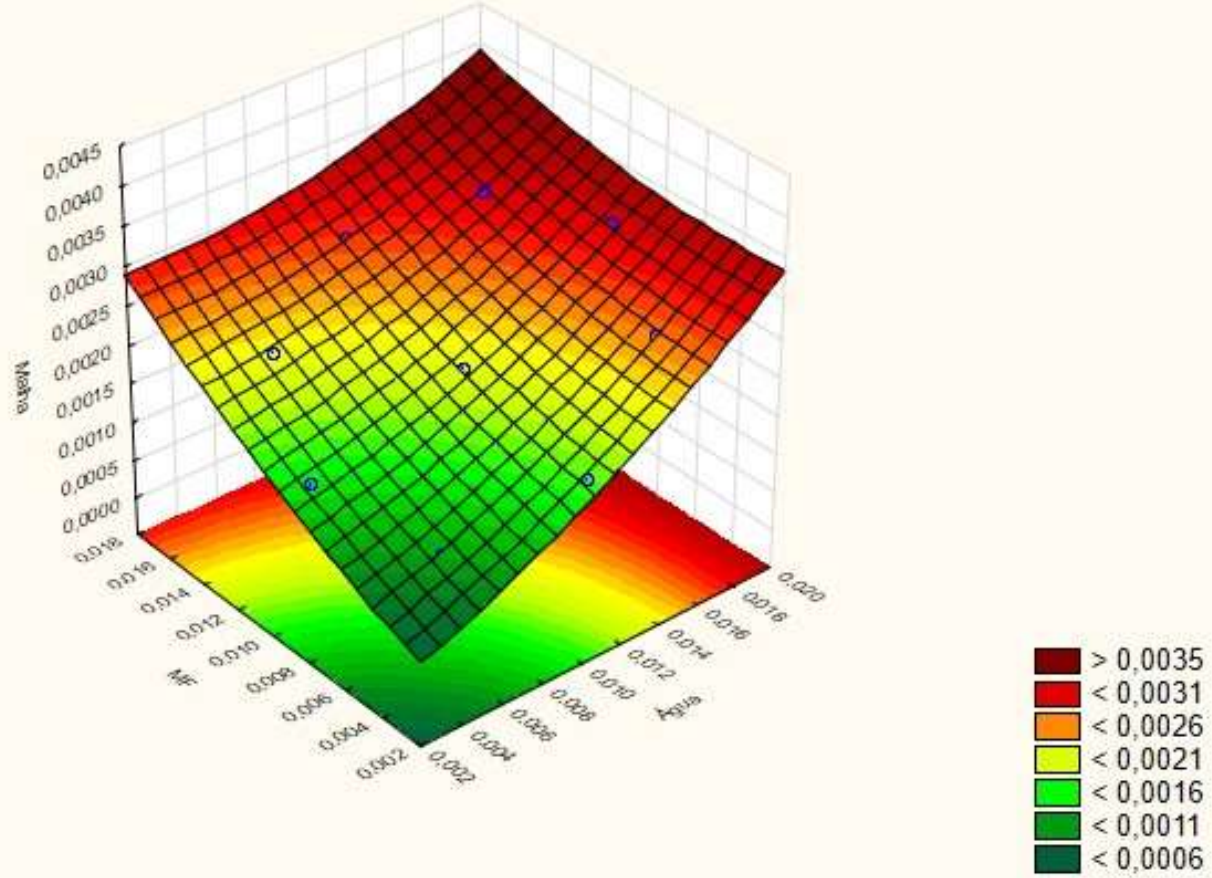

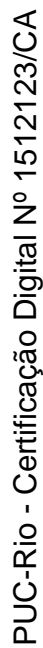

Figura 20 - Superfície de Resposta para a Malha 3.

Curva de Nível; Variável: Malha 3

Fatores $=2$, Blocos $=1$, Experimentos $=9 ;$ MS Residual $=0$

DV: Malha



Figura 21 - Curva de Nível - Malha 3. 
- Malha 4:

Tabela 19 - Tabela ANOVA calculada para os termos da regressão da Malha 4.

\begin{tabular}{|l|c|c|c|c|c|}
\hline \multicolumn{7}{|c|}{ ANOVA - Malha 4 } \\
\hline & $\begin{array}{c}\text { Soma dos } \\
\text { Quadrados }\end{array}$ & $\begin{array}{c}\text { Graus de } \\
\text { Liberdade }\end{array}$ & $\begin{array}{c}\text { Quadrados } \\
\text { Médios }\end{array}$ & $\begin{array}{c}\text { Estatística } \\
\text { F }\end{array}$ & p-valor \\
\hline (1)Água (L) & 0,000001 & 1 & 0,000001 & 435,711 & $2,405 \mathrm{E}-04$ \\
\hline Água(Q) & $5,572 \mathrm{E}-09$ & 1 & $5,572 \mathrm{E}-09$ & 2,287 & 0,227674 \\
\hline (2)MF (L) & $1,617 \mathrm{E}-06$ & 1 & $1,617 \mathrm{E}-06$ & 663,745 & $1,283 \mathrm{E}-04$ \\
\hline MF (Q) & $5,571 \mathrm{E}-09$ & 1 & $5,571 \mathrm{E}-09$ & 2,286 & 0,227719 \\
\hline 1L by 2L & $6,250 \mathrm{E}-08$ & 1 & $6,250 \mathrm{E}-08$ & 25,651 & 0,014859 \\
\hline Erro & $7,310 \mathrm{E}-09$ & 3 & $2,437 \mathrm{E}-09$ & & \\
\hline Total SS & 0,000003 & 8 & & & \\
\hline
\end{tabular}

Gráfico Pareto de Efeitos Padronizados; Variável: Malha 4

Fatores $=2$, Blocos $=1$, Experimentos $=9 ;$ MS Residual $=0$

DV: Malha

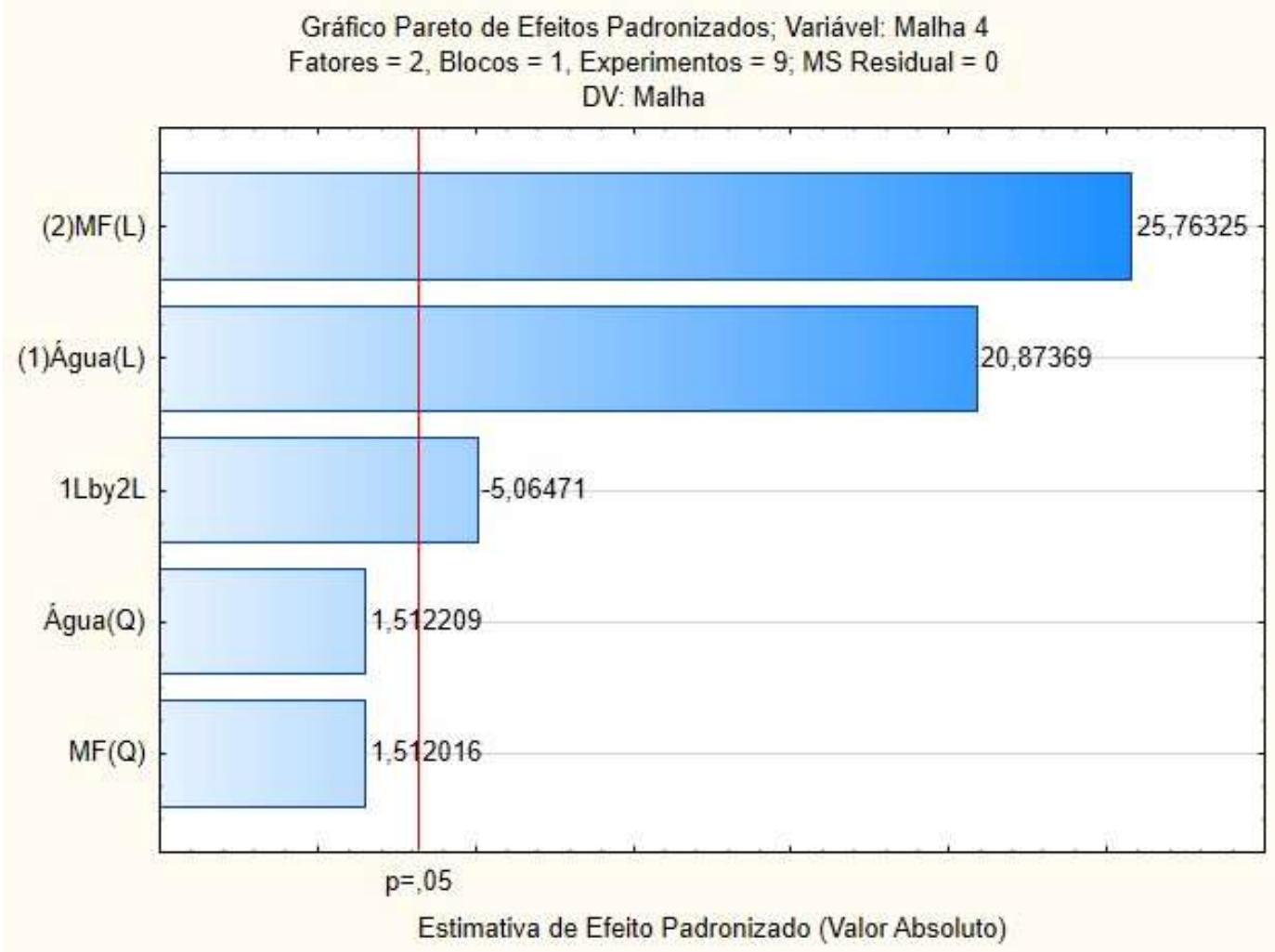

Figura 22 - Gráfico de Pareto das estimativas de efeito padronizado - Malha 4.

A Malha 4, no levantamento inicial da amostra, era a única em que a influência média da incerteza do MF era maior que a da água.

Observando a Figura 22, pode-se observar que a influência estatística dos termos quadráticos para ambas as variáveis é marginalmente significante, no entanto optou-se por manter estas parcelas na análise. Na Figura 23 pode-se ver que o comportamento dos resíduos aponta uma regressão adequada para o modelo. Por fim, gerou-se a Tabela 20 com os resultados do experimento. 


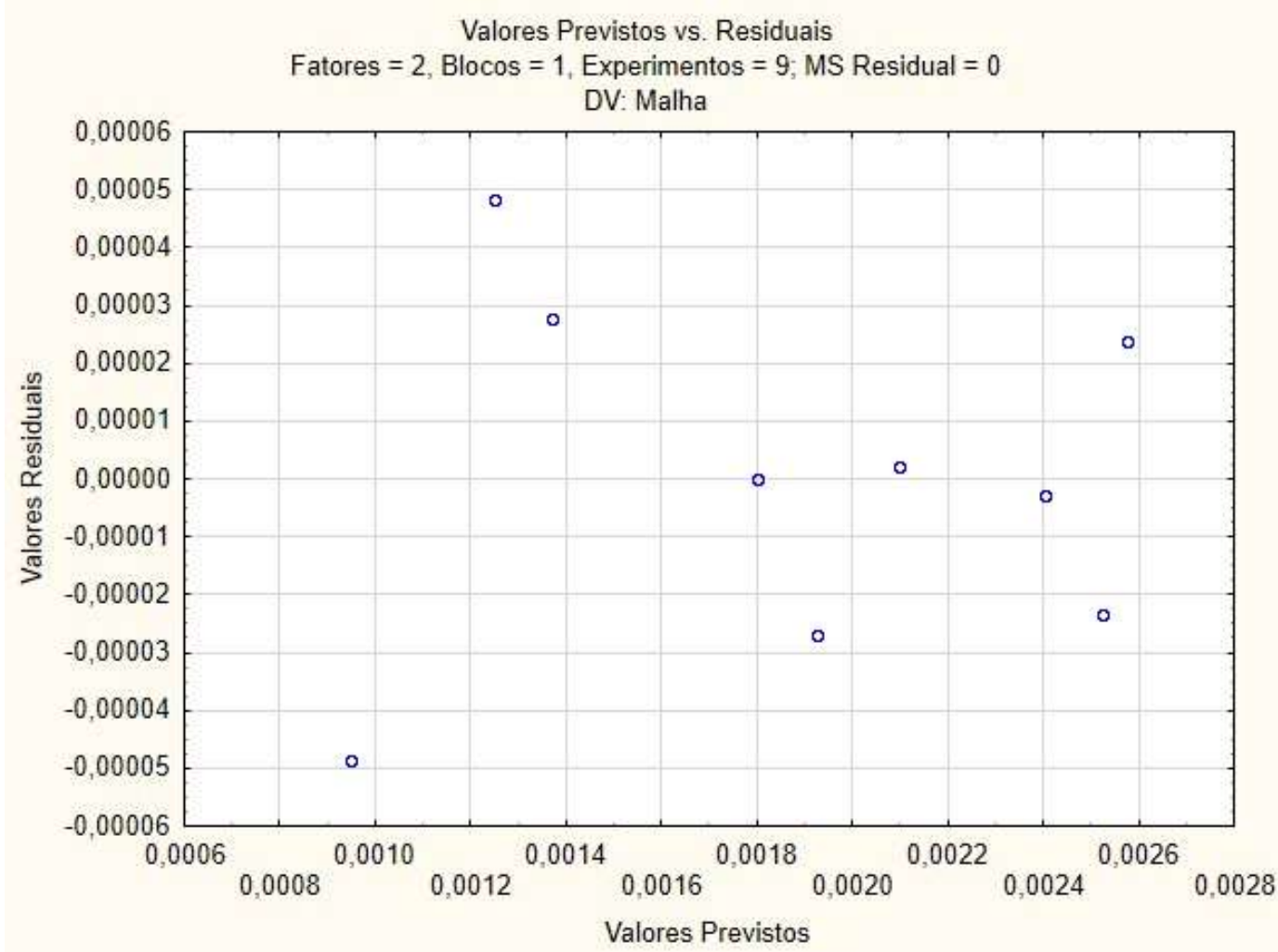

Figura 23 - Gráfico de Resíduos x Valores Previstos para Malha 4.

Tabela 20 - Resultados do experimento para Malha 4.

\begin{tabular}{|c|c|c|c|c|c|c|c|c|c|c|c|c|c|c|}
\hline & & & & \multicolumn{11}{|c|}{ Água } \\
\hline & & & & 0,000000 & 0,001580 & 0,003160 & 0,004740 & 0,006320 & 0,007900 & 0,009480 & 0,011060 & 0,012640 & 0,014220 & 0,015800 \\
\hline & & & & $-2,0$ & $-1,6$ & $-1,2$ & $-0,8$ & $-0,4$ & 0,0 & 0,4 & 0,8 & 1,2 & 1,6 & 2,0 \\
\hline & & & & $-100 \%$ & $-80 \%$ & $-60 \%$ & $-40 \%$ & $-20 \%$ & $0 \%$ & $20 \%$ & $40 \%$ & $60 \%$ & $80 \%$ & $100 \%$ \\
\hline & 000000 & $-2,0$ & $-100 \%$ & $0,002 \%$ & $0,020 \%$ & $0,040 \%$ & $0,061 \%$ & $0,083 \%$ & $0,107 \%$ & $0,133 \%$ & $0,159 \%$ & $0,187 \%$ & $0,217 \%$ & $0,248 \%$ \\
\hline & 0,001816 & $-1,6$ & $-80 \%$ & $0,024 \%$ & $0,040 \%$ & $0,058 \%$ & $0,077 \%$ & $0,097 \%$ & $0,119 \%$ & $0,142 \%$ & $0,167 \%$ & $0,193 \%$ & $0,221 \%$ & $0,249 \%$ \\
\hline & 0,003631 & $-1,2$ & $-60 \%$ & $0,047 \%$ & $0,061 \%$ & $0,077 \%$ & $0,094 \%$ & $0,112 \%$ & $0,132 \%$ & $0,153 \%$ & $0,176 \%$ & $0,200 \%$ & $0,226 \%$ & $0,253 \%$ \\
\hline & 0,005447 & $-0,8$ & $-40 \%$ & $0,071 \%$ & $0,084 \%$ & $0,097 \%$ & $0,112 \%$ & $0,129 \%$ & $0,147 \%$ & $0,166 \%$ & $0,187 \%$ & $0,209 \%$ & $0,232 \%$ & $0,257 \%$ \\
\hline & 0,007263 & $-0,4$ & $-20 \%$ & $0,097 \%$ & \begin{tabular}{|l}
$0,107 \%$ \\
\end{tabular} & $0,119 \%$ & $0,132 \%$ & $0,147 \%$ & $0,163 \%$ & $0,180 \%$ & $0,198 \%$ & $0,219 \%$ & $0,240 \%$ & $0,263 \%$ \\
\hline MF & 0,009078 & 0,0 & $0 \%$ & $0,124 \%$ & $0,133 \%$ & $0,142 \%$ & $0,153 \%$ & $0,166 \%$ & $0,180 \%$ & $0,195 \%$ & $0,212 \%$ & $0,230 \%$ & $0,249 \%$ & $0,270 \%$ \\
\hline & 0,010894 & 0,4 & $20 \%$ & $0,153 \%$ & $0,159 \%$ & $0,167 \%$ & $0,176 \%$ & $0,187 \%$ & $0,198 \%$ & $0,212 \%$ & $0,226 \%$ & $0,243 \%$ & $0,260 \%$ & $0,279 \%$ \\
\hline & 0,012709 & 0,8 & $40 \%$ & $0,183 \%$ & \begin{tabular}{|l}
$0,188 \%$ \\
\end{tabular} & $0,193 \%$ & $0,200 \%$ & $0,209 \%$ & $0,219 \%$ & $0,230 \%$ & $0,243 \%$ & $0,257 \%$ & $0,272 \%$ & $0,289 \%$ \\
\hline & 0,014525 & 1,2 & $60 \%$ & $0,215 \%$ & $0,217 \%$ & $0,221 \%$ & $0,226 \%$ & $0,232 \%$ & $0,240 \%$ & $0,249 \%$ & $0,260 \%$ & $0,272 \%$ & $0,286 \%$ & $0,300^{\circ}$ \\
\hline & 0,016341 & 1,6 & $80 \%$ & $0,248 \%$ & $0,248 \%$ & $0,250 \%$ & $0,253 \%$ & $0,257 \%$ & $0,263 \%$ & $0,270 \%$ & $0,279 \%$ & $0,289 \%$ & & 0,31 \\
\hline & 0,018156 & 2,0 & $100 \%$ & $0,282 \%$ & $0,280 \%$ & $0,280 \%$ & $0,281 \%$ & $0,283 \%$ & $0,287 \%$ & $0,293 \%$ & $0,299 \%$ & 307 & $317 \%$ & $0,3288^{\prime \prime}$ \\
\hline
\end{tabular}

A incerteza média do teor de água para Malha 4 utilizada foi $0,00790 \% \mathrm{v} / \mathrm{v}$ e a incerteza do MF para Malha 4 foi de $0,009078 \mathrm{~m}^{3}$.

Neste caso, em que a parcela de incerteza do MF é maior que a do teor de água, observa-se uma influência semelhante na variação das duas variáveis e no resultado da incerteza da malha. Observa-se que, quando a variação de ambas se aproxima de $80 \%$, ultrapassa-se o limite estabelecido pelo RTM. O comportamento descrito pode ser visto nas Figuras 24 e 25. 
Superficie de Resposta: Variável: Malha 4

Fatores $=2$, Blocos $=1$, Experimentos $=9 ;$ MS Residual $=0$ DV: Malha

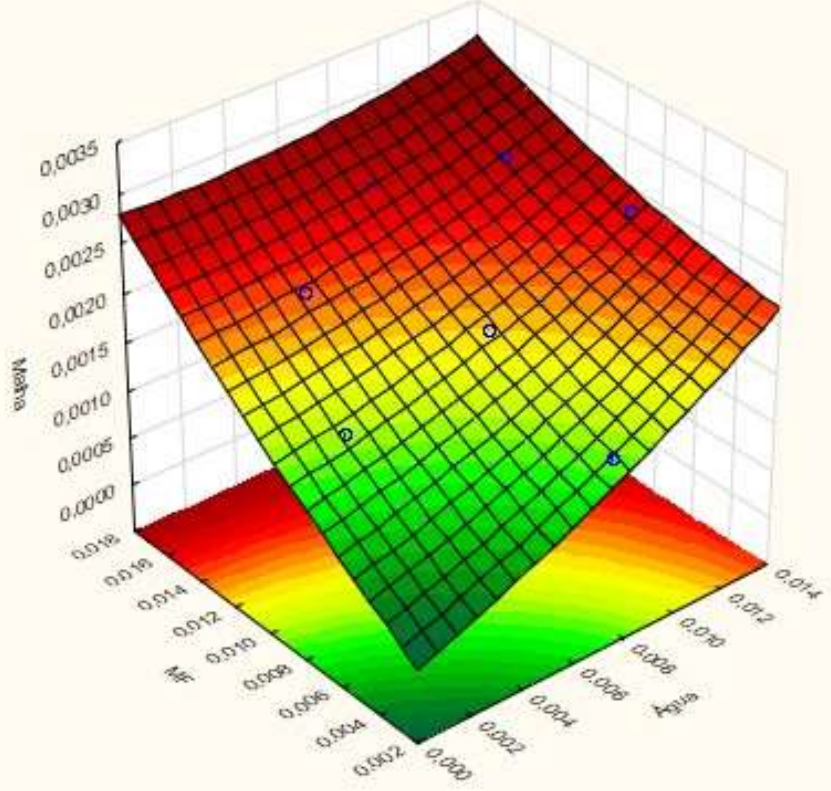

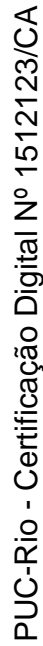

Figura 24 - Superfície de Resposta para a Malha 4.

Curvas de Nivel; Variável: Malha 4

Fatores $=2$, Blocos = 1, Experimentos $=9 ;$ MS Residual $=0$ DV: Malha

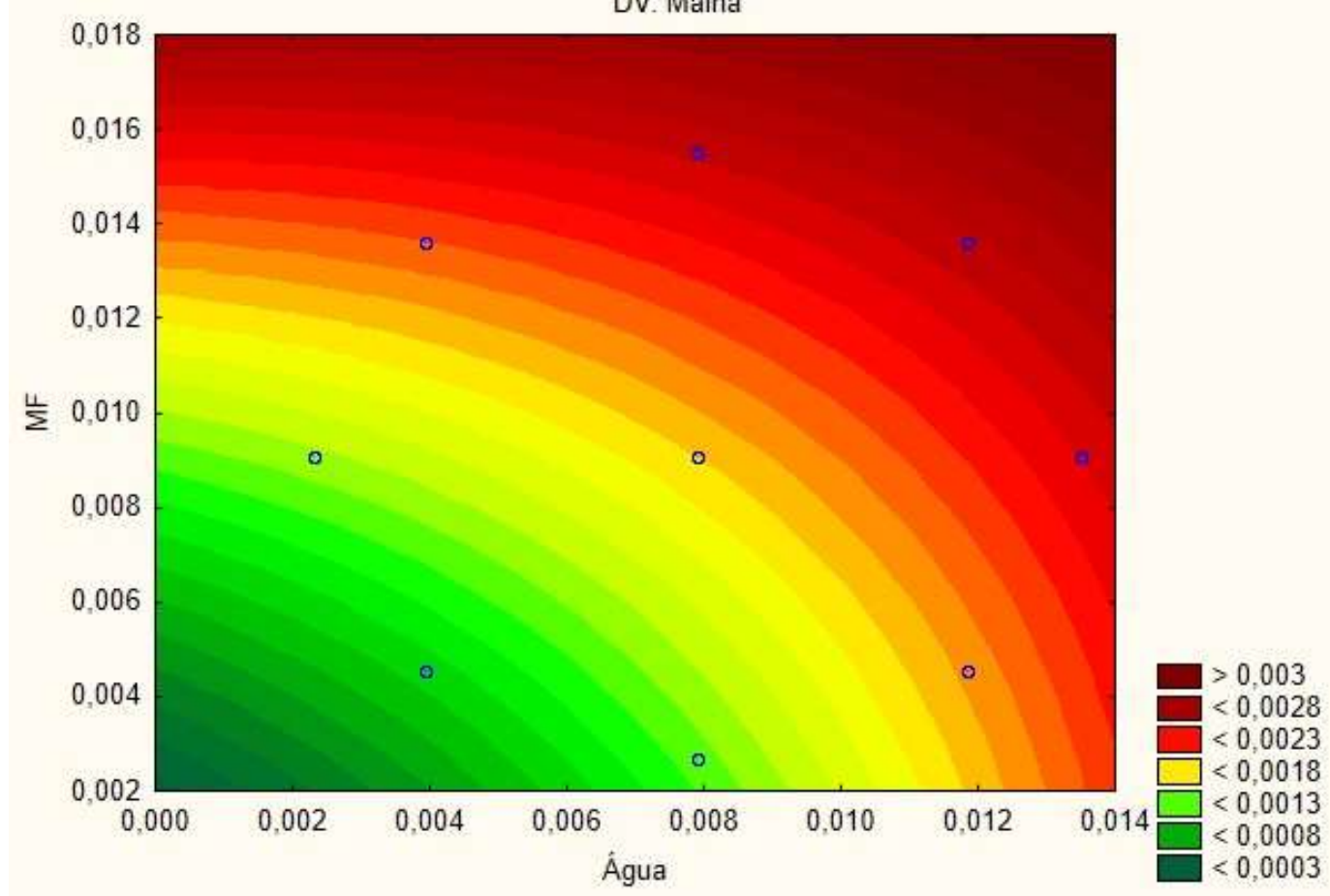

Figura 25 - Curva de Nível - Malha 4. 
- Malha 5:

Tabela 21 - Tabela ANOVA calculada para os termos da regressão da Malha 5.

\begin{tabular}{|c|c|c|c|c|c|}
\hline \multicolumn{7}{|c|}{ ANOVA - Malha 5 } \\
\hline & $\begin{array}{c}\text { Soma dos } \\
\text { Quadrados }\end{array}$ & $\begin{array}{c}\text { Graus de } \\
\text { Liberdade }\end{array}$ & $\begin{array}{c}\text { Quadrados } \\
\text { Médios }\end{array}$ & $\begin{array}{c}\text { Estatística } \\
\text { F }\end{array}$ & p-valor \\
\hline (1)Água (L) & 0,000001 & 1 & 0,000001 & 1032,376 & $6,625 \mathrm{E}-05$ \\
\hline Água (Q) & $9,202 \mathrm{E}-09$ & 1 & $9,202 \mathrm{E}-09$ & 8,949 & 0,058064 \\
\hline (2)MF (L) & $1,062 \mathrm{E}-06$ & 1 & $1,062 \mathrm{E}-06$ & 1032,448 & $6,625 \mathrm{E}-05$ \\
\hline MF (Q) & $9,202 \mathrm{E}-09$ & 1 & $9,202 \mathrm{E}-09$ & 8,949 & 0,058061 \\
\hline 1L by 2L & $2,250 \mathrm{E}-08$ & 1 & $2,250 \mathrm{E}-08$ & 21,881 & 0,018457 \\
\hline Erro & $3,085 \mathrm{E}-09$ & 3 & $1,028 \mathrm{E}-09$ & & \\
\hline Total SS & 0,000002 & 8 & & & \\
\hline
\end{tabular}

Gráfico Pareto de Efeitos Padronizados; Variável: Malha 5

Fatores $=2$, Blocos $=1$, Experimentos $=9 ;$ MS Residual $=0$

DV: Malha

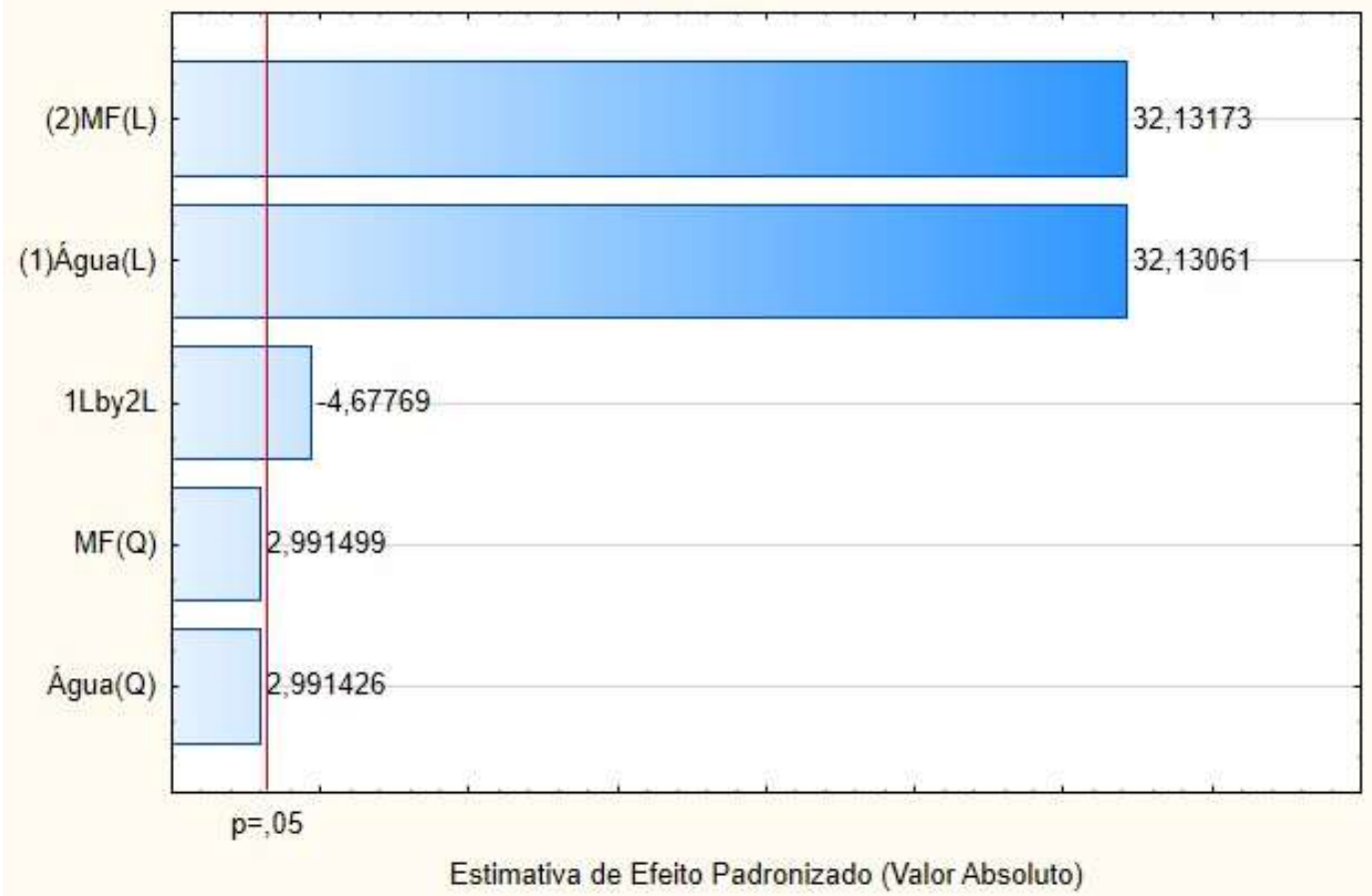

Figura 26 - Gráfico de Pareto das estimativas de efeito padronizado - Malha 5.

Apesar de no levantamento inicial a Malha 5 apresentar uma maior parcela da incerteza para o teor de água, após o tratamento de outliers, conforme Apêndice A, as parcelas de incerteza das duas variáveis ficaram muito próximas.

Observando o gráfico de Pareto da Figura 26 e a tabela ANOVA (Tabela 21) deste caso, optou-se por manter todos os fatores do modelo. Após isso, pode-se observar a distribuição esperada dos resíduos na Figura 27 e os resultados na Tabela 22 . 


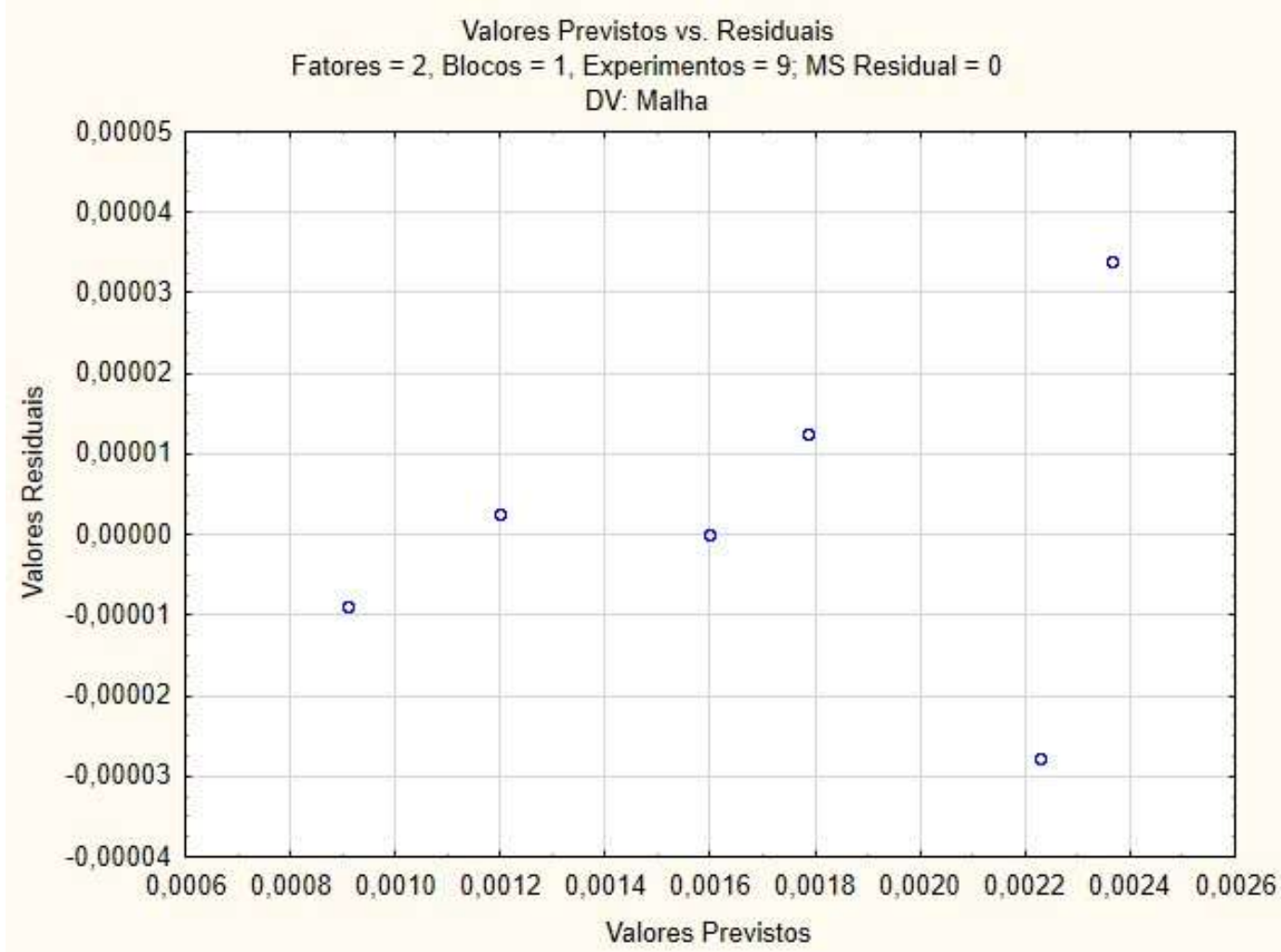

Figura 27 - Gráfico de Resíduos x Valores Previstos para Malha 5.

Tabela 22 - Resultados do Experimento para Malha 5.

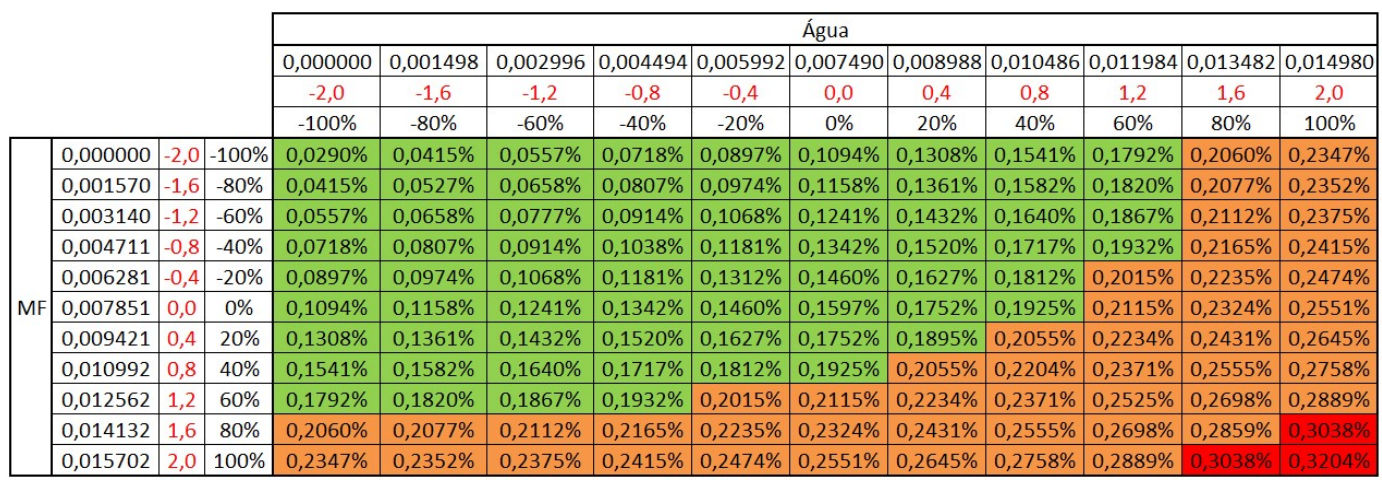

Os resultados indicaram variações de $35 \%$, conforme Tabela 23.

A incerteza média do teor de água para Malha 5 utilizada foi $0,007490 \% \mathrm{v} / \mathrm{v}$ e a incerteza do MF para Malha 5 foi de $0,007851 \mathrm{~m}^{3}$.

O comportamento desta malha é muito parecido com a da Malha 4. Com variação de $80 \%$ de ambas as variáveis, aproxima-se do limite de $0,3 \%$ da incerteza da malha. Este comportamento pode ser observado nas Figuras 28 e 29. 
Superficie de Resposta: Variável: Malha 5

Fatores $=2$, Blocos $=1$, Experimentos $=9 ;$ MS Residual $=0$ DV: Malha

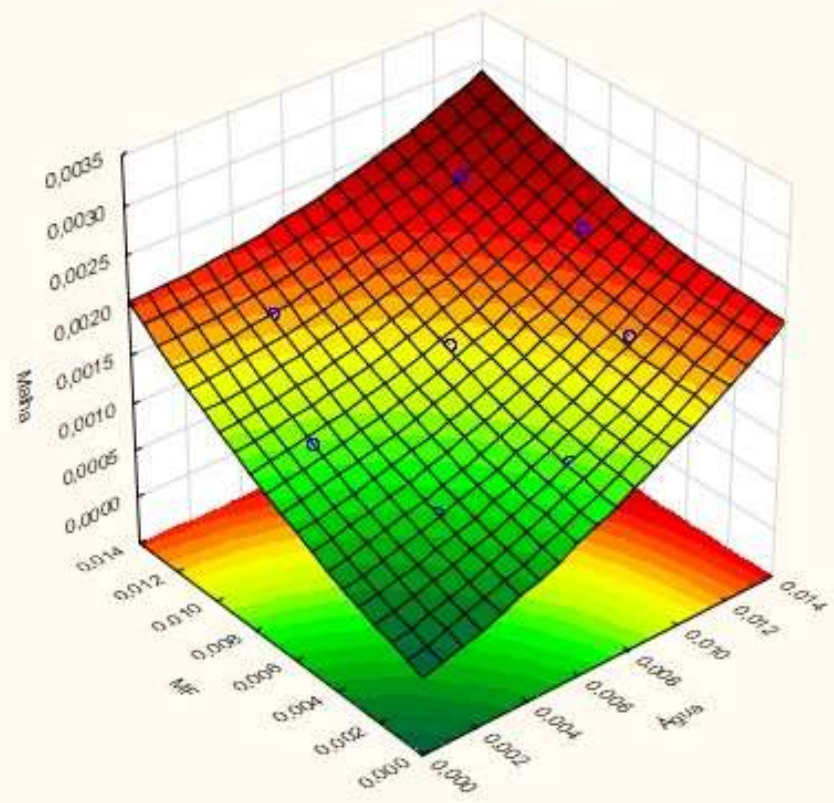

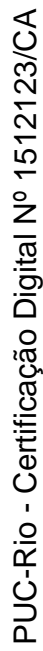

Figura 28 - Superfície de Resposta para a Malha 5.

Curva de Nível; Variável: Malha 5

Fatores $=2$, Blocos $=1$, Experimentos $=9 ;$ MS Residual $=0$ DV: Malha

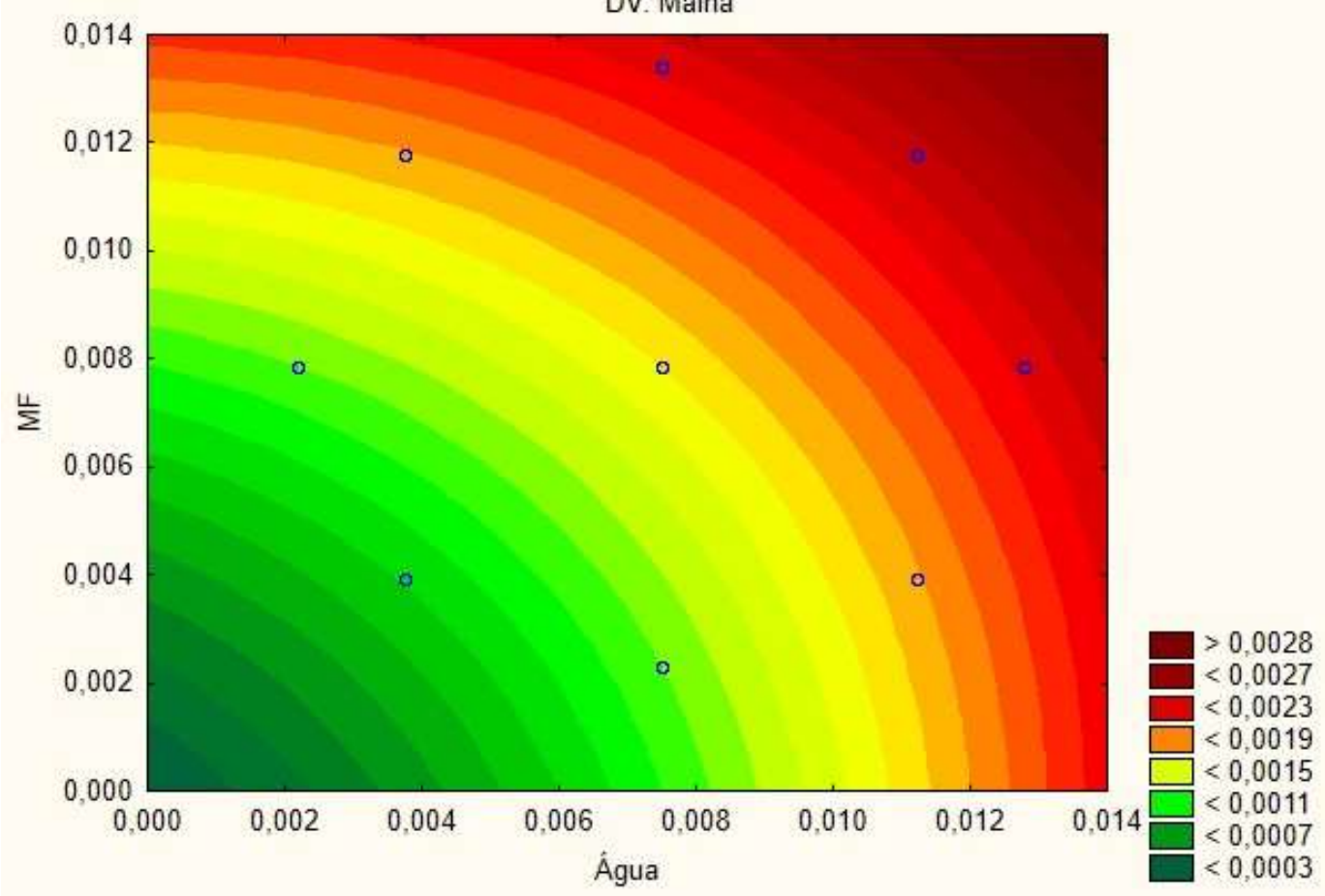

Figura 29 - Curva de Nível - Malha 5. 
Tabela 23 - Resultados do Experimento para Malha 5 com variação de $35 \%$.

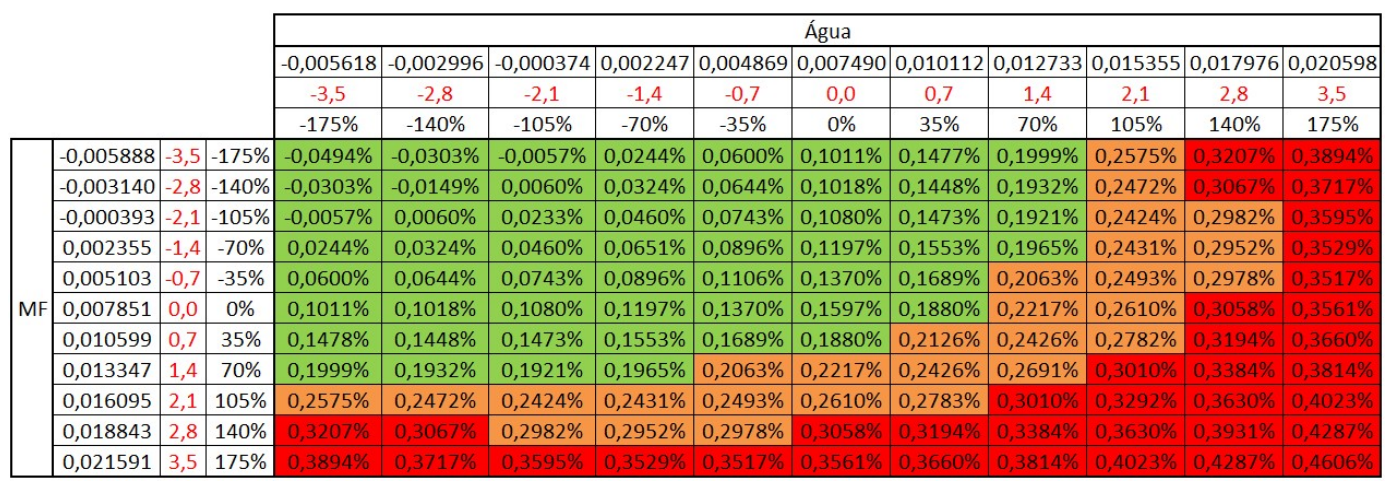

- Malha 6:

Tabela 24 - Tabela ANOVA calculada para os termos da regressão da Malha 6.

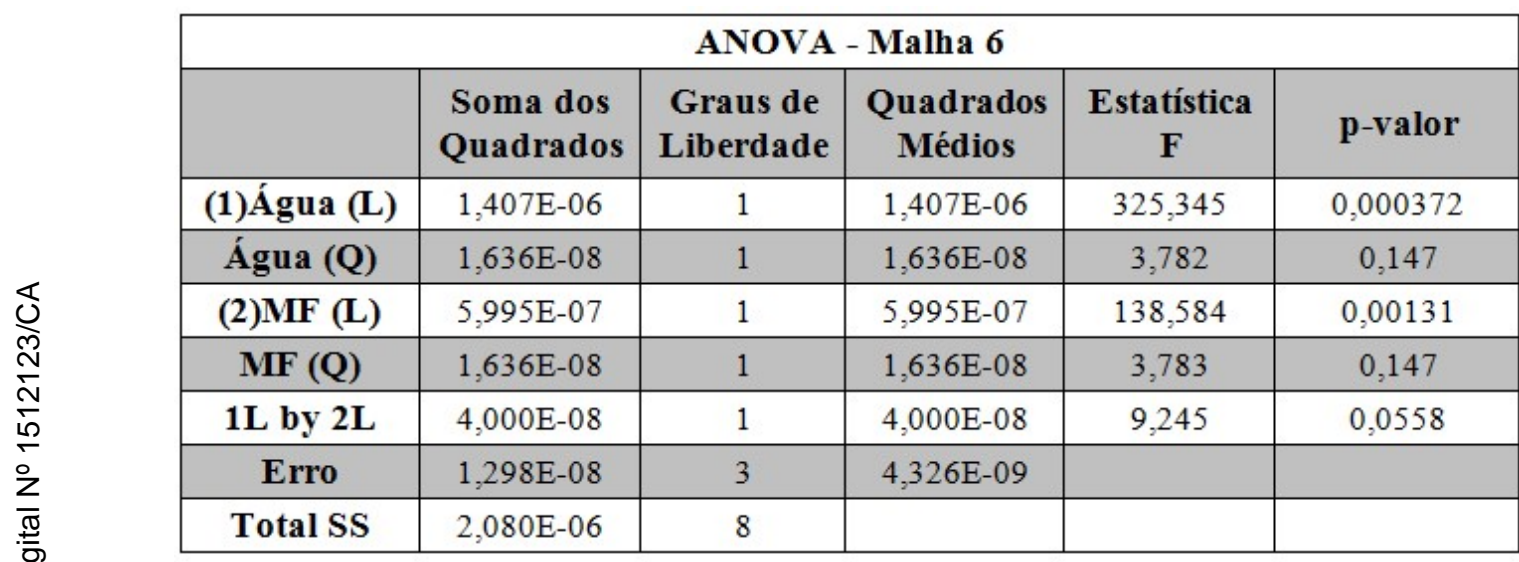

Gráfico Pareto de Efeitos Padronizados; Variável: Malha 6

Fatores $=2 ;$ Blocos $=1 ;$ Experimentos $=9 ; \mathrm{MS}$ Residual $=0$ DV: Malha

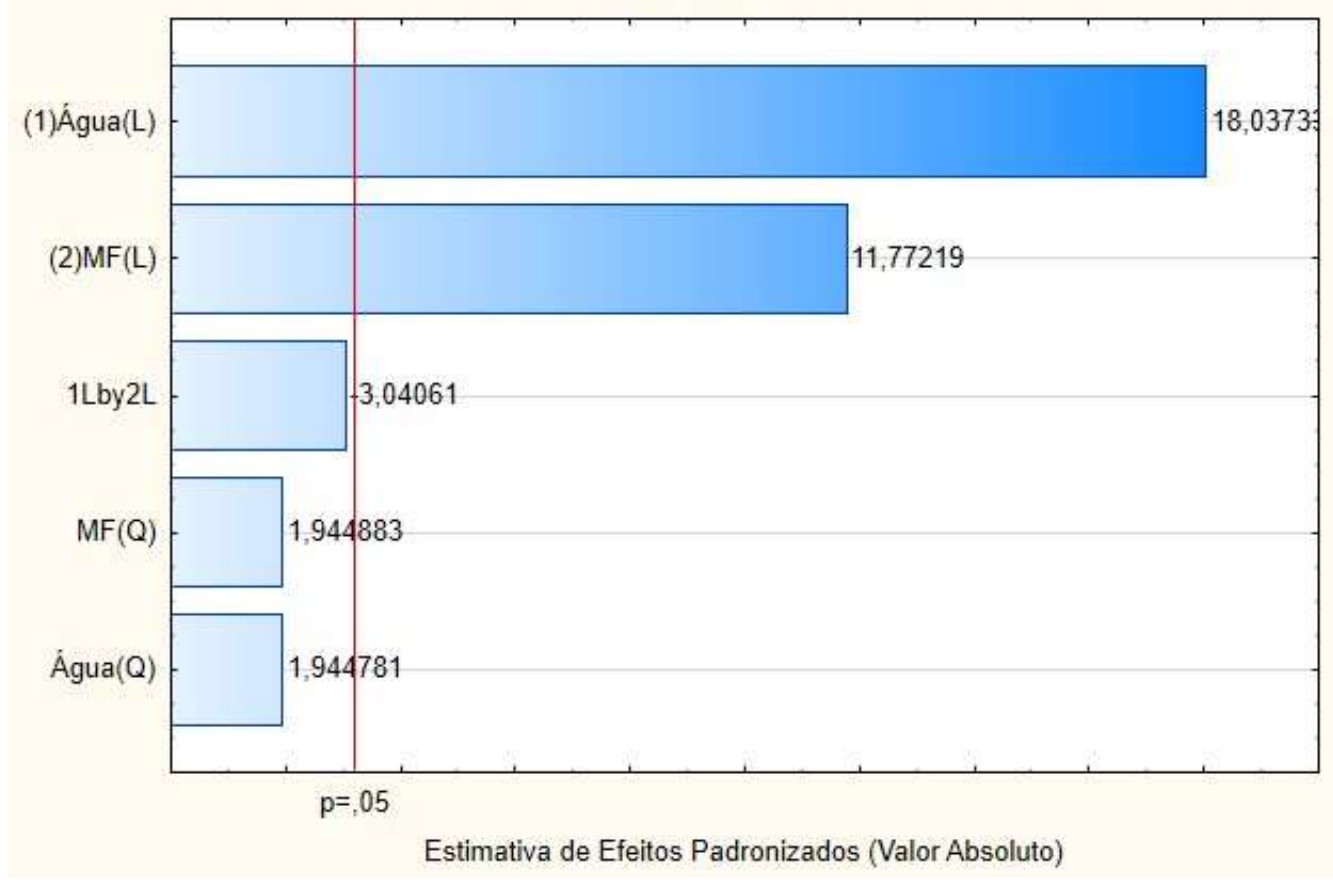

Figura 30 - Gráfico de Pareto das estimativas de efeito padronizado - Malha 6. 
Na Figura 30 pode-se observar que os termos lineares de ambas as variáveis são estatisticamente significativos e que os termos quadráticos e o termo de interação das variáveis foram mantidos, apesar de sua relevância marginal, como pode ser visto na Tabela 10 na coluna Malha 6. Analisa-se, então, o gráfico de resíduos da Figura 31 e este está com o comportamento esperado para um modelo adequado.

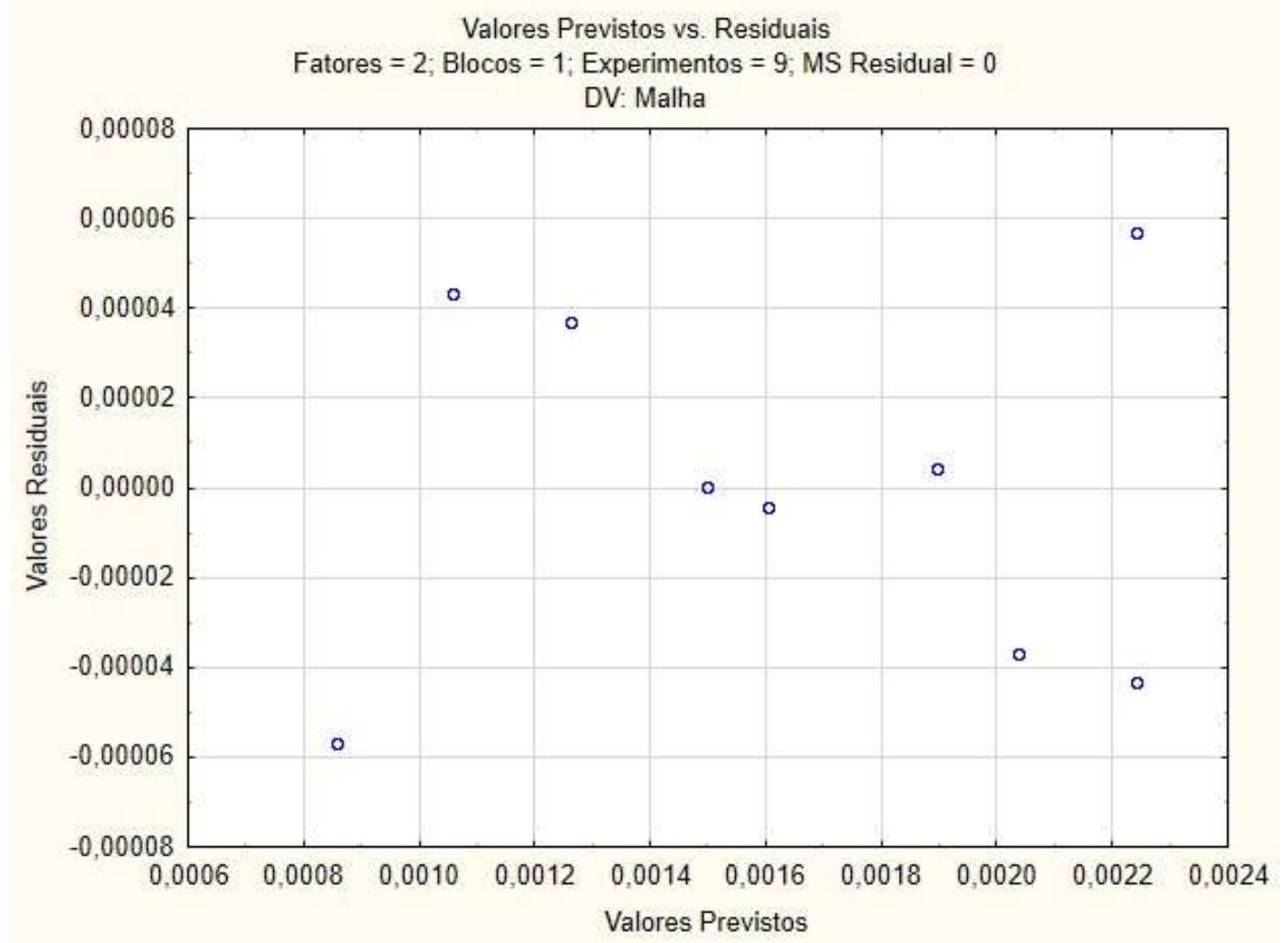

Figura 31 - Gráfico de Resíduos x Valores Previstos para Malha 6.

Tabela 25 - Resultados do Experimento para Malha 6.

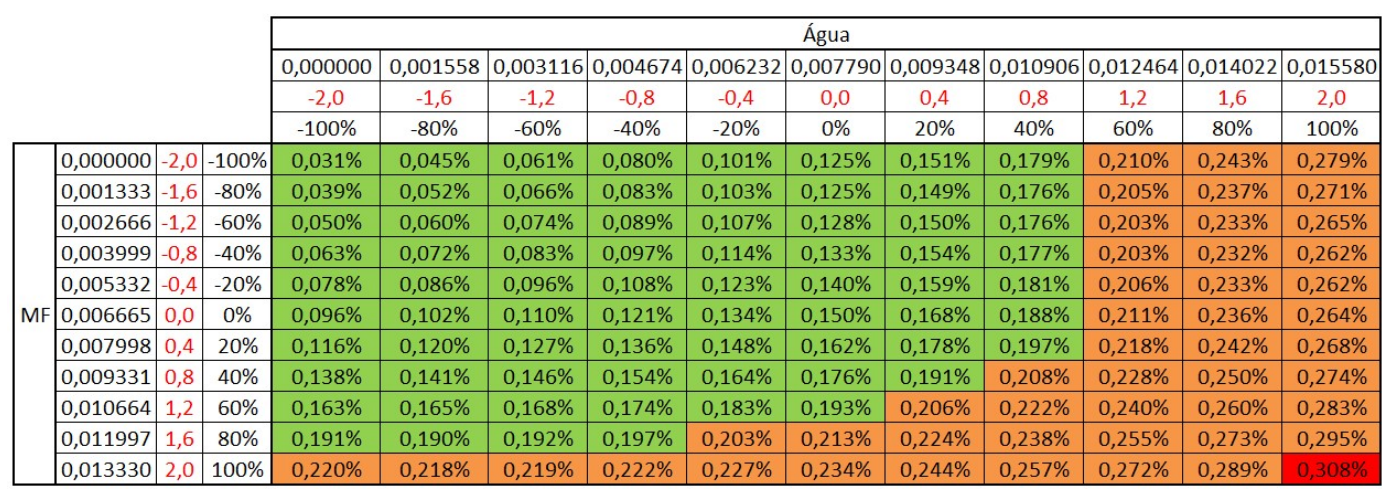


Tabela 26 - Resultados do Experimento para Malha 6 com variação de $35 \%$.

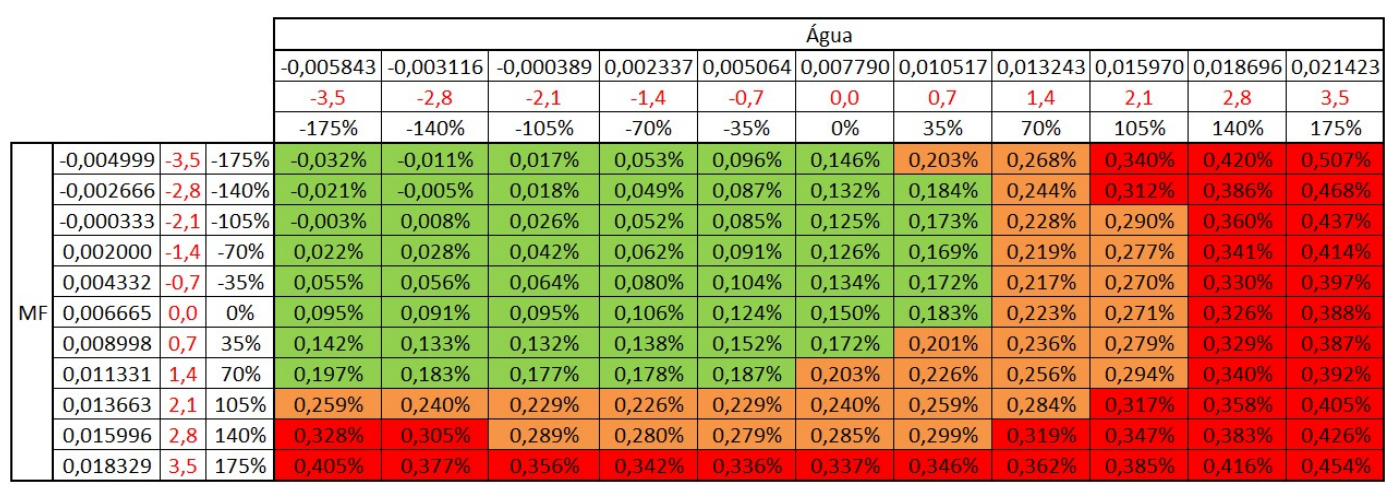

A incerteza média do teor de água para Malha 6 utilizada foi $0,007790 \% \mathrm{v} / \mathrm{v}$ e a incerteza do MF para Malha 6 foi de $0,006665 \mathrm{~m}^{3}$. Foi calculada uma variação de $\pm 100 \%$ para cada variável, de 20 em $20 \%$. Ilustra-se, também, a Tabela 26, com os resultados para variação de $35 \%$ das variáveis, a fim de visualizar melhor o efeito da curvatura da superfície de resposta.

Neste caso, pode-se observar que com uma variação perto de $100 \%$ de ambas as variáveis, atinge-se a incerteza limite da malha, conforme Figuras 32 e 33.

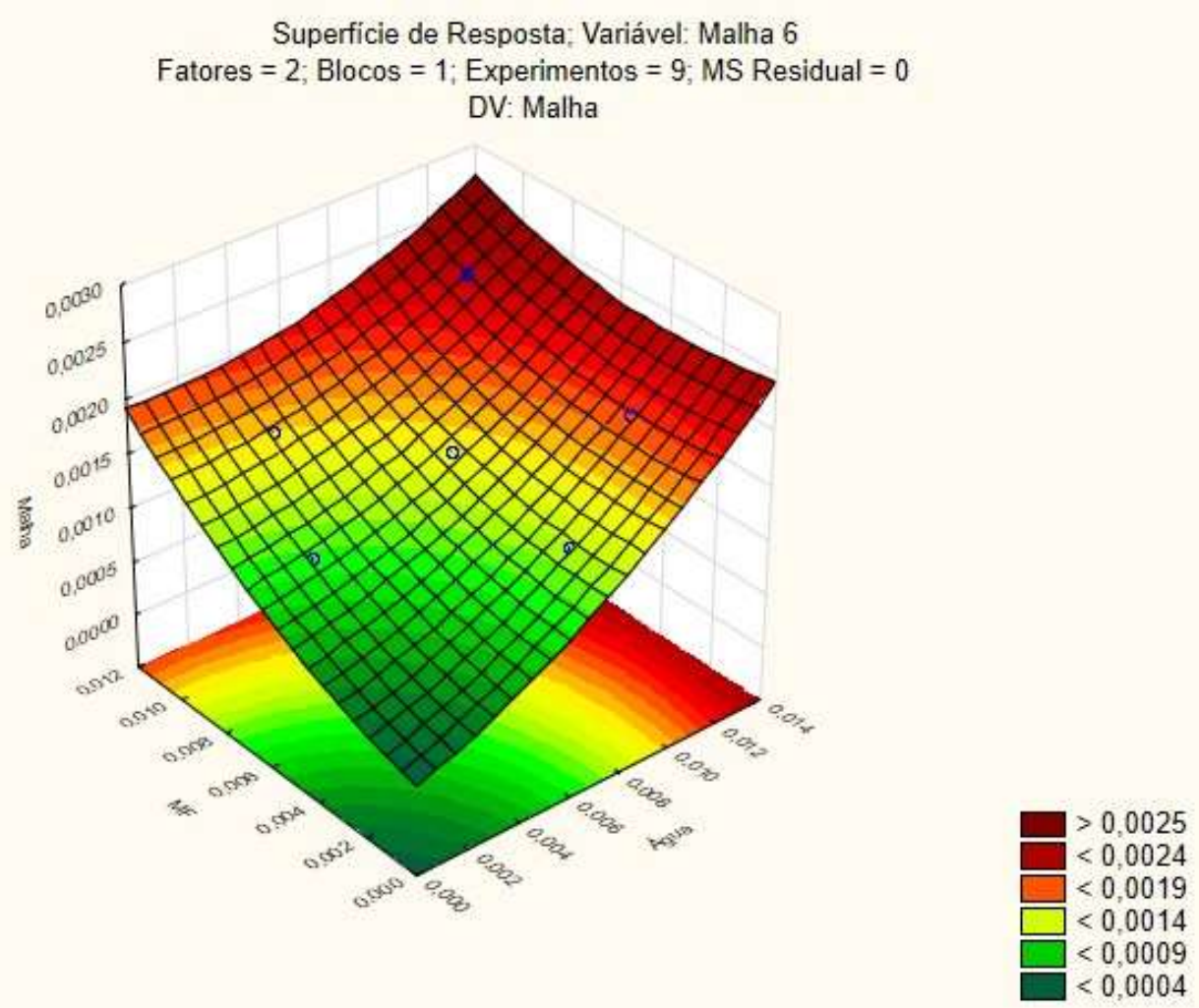

Figura 32 - Superfície de Resposta para a Malha 6. 


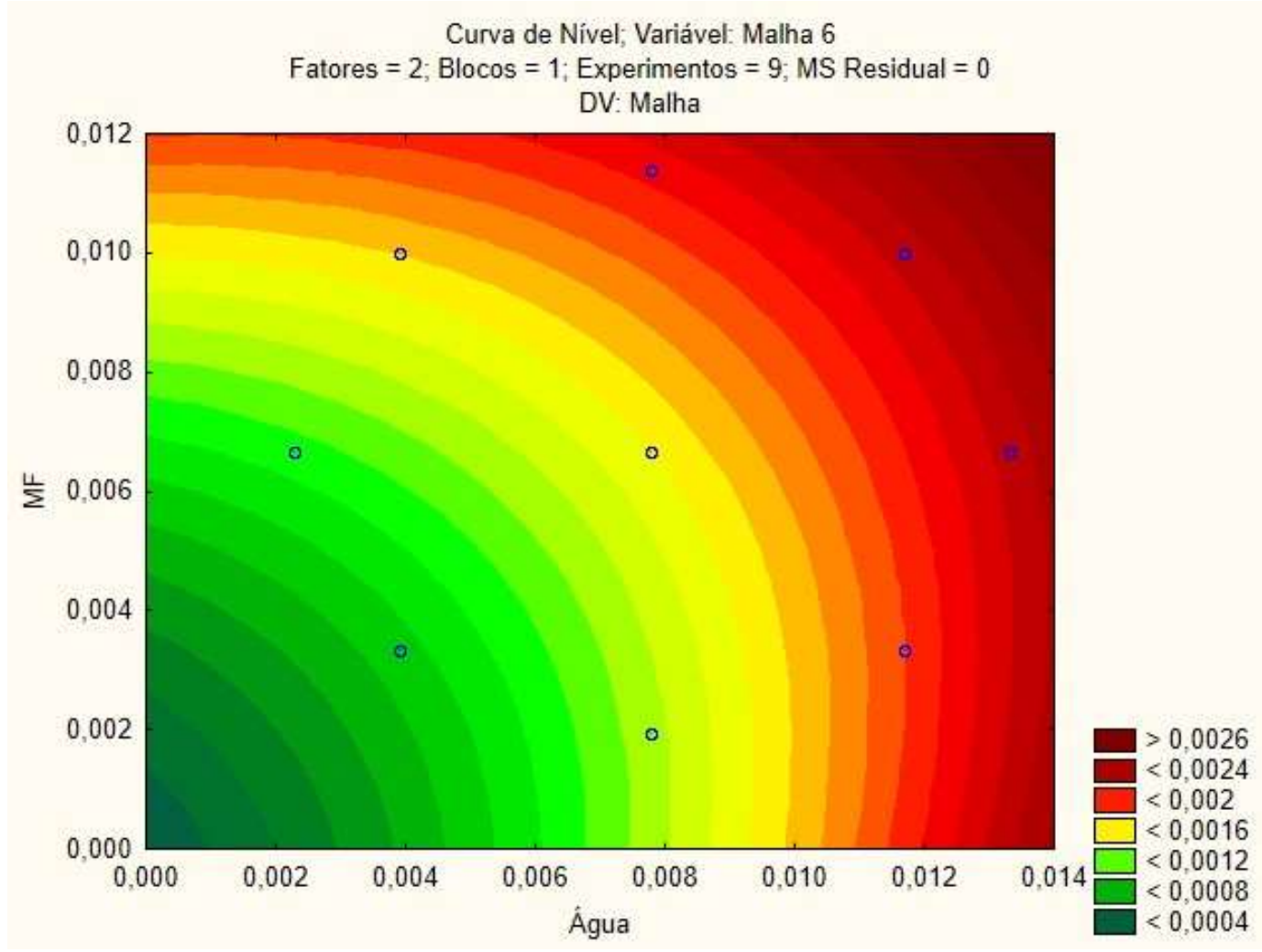

Figura 33 - Curva de Nível - Malha 6.

\section{3}

\section{Análise dos experimentos}

Analisando os experimentos, concluiu-se que, fixando os demais fatores de contribuição da incerteza, pode-se simular a influência dos valores tanto da incerteza expandida da análise do teor de água quanto da incerteza expandida do fator do medidor na incerteza da malha.

Neste caso, não convém analisar os pontos críticos encontrados, pois os mesmos apresentam valores negativos, situação metrologicamente impossível.

Algumas observações importantes:

- $\quad$ Exceto nas Malha 4 e 5, a influência da incerteza do MF foi inferior à influência da incerteza do teor de água;

- Nas Malhas 4 e 5 as influências das duas variáveis foram estatisticamente similares;

- Variações pequenas da incerteza expandida de água podem levar a ultrapassar o limite da incerteza da malha, como pode ser observado nas Malhas 2 e 3 na faixa de $40 \%$. 
- Mantidos os patamares da incerteza dos teores de água atuais, podese variar em até $80 \%$ a incerteza do MF sem atingir o valor limite para incerteza da malha.

Observa-se que, com variações da incerteza do teor de água abaixo de $100 \%$, pode-se ultrapassar os $0,3 \%$ permitidos pelo RTM para incerteza da malha, sendo que na Malha 3, com 60 \% de variação ultrapassou-se a incerteza limite para todas as incertezas de MF simuladas. Ou seja, as médias atualmente encontradas para incerteza de teor de água (que neste trabalho foram obtidas com a utilização do método Karl Fischer Coulométrico) são adequadas para manter a incerteza dentro da permitida pelo RTM, porém com pouca variação pode-se exceder este limite. Um estudo para redução desta incerteza (voltado para melhorar a incerteza de repetibilidade de cada laboratório, por exemplo) diminuiria esta possibilidade. Como o método por Centrifugação apresenta incertezas mais altas, este não se mostra adequado para este tipo de malha fiscal, devendo ser utilizado somente para valores de teor de água mais altos e malhas de apropriação ou operacionais, ou em malhas em que a incerteza do MF seja extremamente menor e o comportamento das demais parcelas seja similar ou menor do que o apresentado nesta dissertação.

Em todos os casos, a influência da incerteza do fator do medidor foi inferior ou similar à incerteza do teor de água. O RTM define que a variação entre os fatores de calibração das corridas realizadas seja de no máximo 0,05 \% (repetibilidade) e a variação comparada com a do MF anterior seja no máximo 0,25\% (critério-limite de calibração permitido pela ANP), o que se mostra muito conservador em uma análise que considera unicamente o atendimento da incerteza máxima da malha (não considerando outras questões como mudança de comportamento do medidor em faixas diferentes de MF ou que a mudança de MF pode indicar necessidades de manutenção do mesmo).

A repetibilidade do medidor, mesmo sendo um insumo da incerteza, é relacionada diretamente ao cálculo de erro de medição. Não há a necessidade de se ter dois critérios de avaliação do equipamento, um de incerteza e um de "erro máximo admissível". A incerteza se mostra mais adequada, pois a mesma acompanha o instrumento dinamicamente em seu uso, enquanto a avaliação da repetibilidade é estática, uma característica do momento de calibração.

Como o RTM prevê a periodicidade das calibrações dos instrumentos fiscais e cada calibração gera uma incerteza para o instrumento, sugere-se para uma futura 
revisão do RTM a adoção de um critério limite de incerteza ao invés de um critério de repetibilidade para os medidores ultrassônicos, ou utilizar como critério somente a incerteza da malha.

Caso haja a diminuição da parcela de incerteza do teor de água, pode-se cogitar até uma maior incerteza para a calibração dos medidores ultrassônicos. Plataformas com processos instáveis devido ao comportamento dos poços produtores ou por quebras de equipamentos críticos apresentam grande dificuldade de calibrar estes medidores dentro da faixa limite de $0,25 \%$, muitas vezes tendo que restringir poços produtores ou ter perdas de produção para conseguir a estabilização necessária de vazão para efetuar a calibração dentro dos requisitos definidos pelo Regulamento vigente. Uma maior flexibilidade destes limites geraria menos perdas e não diminuiria a qualidade da medição, visto que o critério limite de $0,3 \%$ continuaria sendo atendido. 


\section{6 \\ Conclusões e recomendações}

\section{1 \\ Conclusões}

Alinhado aos objetivos desta dissertação, define-se que os métodos mais adequados para determinação do teor de água em óleo são: análise por centrifugação, Karl Fischer volumétrico e Karl Fischer coulométrico. Os três métodos podem ser realizados nos laboratórios do ambiente offshore, com espaço reduzido e em curto período de tempo. Analisando as normas referentes a estes métodos e seus cálculos de incerteza, observa-se que a incerteza do método de centrifugação tende a ser maior, o que pode levar a incerteza da malha a ultrapassar o valor de 0,3 \% definido pelo RTM, sendo mais adequada a utilização do Karl Fischer para a faixa de teor de água até $1 \%$.

Utilizando o planejamento de experimentos, conclui-se que, fixando os demais fatores de contribuição da incerteza, é possível simular a influência da análise de teor de água e do fator do medidor na incerteza da malha de medição de vazão de óleo utilizando o princípio ultrassônico, sendo a influência do fator do medidor menor ou similar à da água nos casos estudados. Mantidos os valores atuais da incerteza do teor de água, pode-se variar até $80 \%$ a incerteza expandida do fator do medidor sem ultrapassar o limite estabelecido pela regulamentação para a incerteza da malha.

Sugere-se que em uma futura revisão do RTM, este adote um critério limite de incerteza para a calibração dos medidores ultrassônicos, ao invés de critérios de repetibilidade.

Nas condições atuais, o método Karl Fischer atende aos requisitos do RTM. Caso seja necessário reduzir a incerteza deste, pode-se fazer um estudo para melhorar a incerteza da repetibilidade do laboratório, fator com maior influência na incerteza deste método. 


\section{2 \\ Recomendações para trabalhos futuros}

Sugere-se para trabalhos futuros:

- Realizar estudo similar para outras malhas de medição de vazão de óleo, utilizando ou não o princípio ultrassônico, a fim de comparar os efeitos de diferentes temperaturas, pressões, viscosidade e massas específicas;

- $\quad$ Analisar a influência da variação dos demais fatores na incerteza da malha de vazão de óleo ultrassônico; e

- Realizar estudo para situações em que o BSW esteja com valores elevados.

Esta pesquisa contribuiu para que se evite utilizar métodos de análise de teor de água que gerem incertezas para malha de medição superiores às permitidas pelo RTM, podendo gerar não conformidades e multas perante a ANP, utilizando a análise de dados reais. Além disto, este trabalho pode ser utilizado como um dos subsídios para modificar o método de avaliação da calibração pelo RTM e para aprovação de outros métodos de análise do teor de água no óleo.

Por último, este trabalho difunde as metodologias de superfície de resposta e de planejamento de experimentos. 


\section{7 Referências bibliográficas}

ASSOCIAÇÃO BRASILEIRA DE NORMAS TÉCNICAS - ABNT-14236/02: Produtos de Petróleo e Materiais Betuminosos - Determinação do Teor de Água por Destilação. Rio de Janeiro, 2002. 8 p.

ABNT-NBR 14647/01. Produtos de Petróleo - Determinação da Água e Sedimentos em Petróleos e Óleos Combustíveis pelo Método de Centrifugação. Rio de Janeiro, 2001. 9 p.

AGÊNCIA NACIONAL DO PETRÓLEO, GÁS NATURAL E BIOCOMBUSTÍVEIS. INSTITUTO NACIONAL DE METROLOGIA, QUALIDADE E TECNOLOGIA. Resolução Conjunta ANP/INMETRO n $^{\circ}$ 001, de 10 de junho de 2013. Dispõe sobre o regulamento técnico de medição de petróleo e gás natural. Diário Oficial da República Federativa do Brasil, Brasília, DF. 2013.

AGÊNCIA NACIONAL DO PETRÓLEO, GÁS NATURAL E BIOCOMBUSTÍVEIS. Resolução ANP n 52 de 26 de dezembro de 2013. Dispõe sobre o Regulamento Técnico de Implementação de Resultados de Análises Físico-Químicas para as Medições Subsequentes de Petróleo e Gás Natural. Diário Oficial da República Federativa do Brasil, Brasília, DF. 2013.

Site institucional. Disponível em: <http://www.anp.gov.br/?pg=70721\&m=\&t1=\&t2=\&t3=\&t4=\&ar=\&ps=\&147 0053015175>. Acesso em: 12 jun 2016.

ALENCAR, A. L. S de, et al. Quality versus economical aspects in determination of water in crude oils: Centrifuge method or potentiometric Karl Fischer titration. Petroleum Science and Technology, v. 34, n. 3, p. 287-294, 2016. 
AMERICAN PETROLEUM INSTITUTE API/MPMS 10.1/02. Standard Test Method for Sediment in Crude Oils and Fuel Oils by the Extraction Method, Second Edition. Washington D. C., 2002. 5 p.

API/MPMS 10.4/99. Determination of Water and Sediment in Crude Oil by Centrifuge Method (Field Procedure). Washington D. C., 1999. $17 \mathrm{p}$.

. API/MPMS 10.7/02. Standard Test Method for Water in Crude Oil by Karl Fischer. Titration (Potentiometric) (ANSI/ASTM D4377) (IP 356). Washington D. C., 2002. 6 p.

. API/MPMS 10.9/2013. Standard Test Method for Water in Crude Oils by Coulometric Karl Fischer Titration, Manual of Petroleum Measurements Standards. Washington D. C., 2013. 6 p

API/MPMS 11.1/2007. Temperature and Pressure Volume Correction Factors for Generalized Crude Oils, Refined Products, and Lubricating Oils. Washington D. C., 2007. 187 p.

. API/MPMS 20.1/1993. Allocation Measurement of Oil and Natural Gas. Washington D. C., 1993. 67 p.

ARANTES, W. F. Avaliação metrológica da comparação interlaboratorial da calibração de medidores ultra-sônicos. Dissertação de Mestrado Departamento de Engenharia Mecânica. Rio de Janeiro: Pontifícia Universidade Católica do Rio de Janeiro, 2007. 142 p.

AMERICAN SOCIETY FOR TESTING AND MATERIALS - ASTM D 4007/08: Standard Test Method for Water and Sediment in Crude Oil by the Centrifuge Method (Laboratory Procedure). West Conshohocken, 2008.

- ASTM D 4928/12 Standard method for water in crude oils by coulometric Karl Fischer titration. West Conshohocken, 2012. 
BIAZON, C. L.; JESUS, V. C. B. M.; DE OLIVEIRA, E. C. Metrological analysis by measurement uncertainty of water and sediment in crude oil. Petroleum Science and Technology, v. 33, n. 3, p. 344-352, 2015.

COSTA E SILVA, C B.; ANDRADE, L. A. Estado atual da medição de BSW na Petrobras. Relatório Técnico. Petrobras, Brasil, 2013.

CUNICO, M. W. M. et al. Planejamento Fatorial: Uma ferramenta estatística valiosa para a definição de parâmetros experimentais empregados na pesquisa científica. Curitiba: Visão Acadêmica, v. 9, n. 1, 2008.

DUNN, William C. Fundamentos de Instrumentação Industrial e Controle de Processos. São João del-Rei: Bookman Editora, 2013.

EURACHEM. Quantifying uncertainty in analytical measurement (2nd ed.). Helsinki, Finland: EURACHEM. International Organization for Standardization. (1988). Petroleum and liquid petroleum products calculation of oil quantities - part 2: Dynamic measurement. Method ISO 4267-2, 2000.

INSTITUTO NACIONAL DE METROLOGIA, QUALIDADE E TECNOLOGIA - INMETRO. Site institucional. Disponível em <http://www.inmetro.gov.br/inmetro/oque.asp>. Acesso em 12 jun 2016.

INTERNATIONAL ORGANIZATION FOR STANDARDIZATION. Guide to the expression of uncertainty in measurement (GUM).Geneva: ISO, 1995.

LEMOS, A. L., SILVA, J. M. C., DANTAS A. N. S., \& LOPES, G. S. Utilização de experimento didático para aprendizado de planejamento experimental em Química. In: VII CONNEPI-Congresso Norte Nordeste de Pesquisa e Inovação. 2012.

LIU, Jin. Investigation of trace amounts of gas on microvave water-cut measurement. Tese de Doutorado. Texas: Texas A\&M University, 2006. 
MYERS, R. H.; MONTGOMERY, D. C.; ANDERSON-COOK, C. M. Response surface methodology: process and product optimization using designed experiments. John Wiley \& Sons, 2016.

NETO, B. B.; SCARMINIO, I. S.; BRUNS, R. E. Como fazer experimentos. Campinas: Editora da Unicamp, 2003.

OLIVEIRA, E. C. de. Otimização do critério de aceitação na calibração de instrumentos para medição de transferência de custódia de gás natural. São Paulo: ENQUALAB, 2006.

SALGADO, A. L. Análise Experimental da Variabilidade Induzida Por Acidentes de Linha na Medição de Vazão Ultrassônica Por Tempo de Trânsito. Dissertação de Mestrado. Vitória: Universidade Federal do Espírito Santo, 2009.

SANTOS, V. C. M. A medição fiscal dos volumes de petróleo: medidores ultrassônicos. Dissertação de Mestrado. Niterói: Universidade Federal Fluminense, 2011.

TEÓFILO, Reinaldo F.; FERREIRA, Márcia MC. Quimiometria II: planilhas eletrônicas para cálculos de planejamentos experimentais, um tutorial. Química Nova, v. 29, n. 2, p. 338, 2006.

THOMAS, J. E. Fundamentos de engenharia de petróleo. Rio de Janeiro: Interciência, 2001. 


\section{APÊNDICE A - Teste de Normalidade das Amostras}

Para testar se as amostras seguem uma distribuição Normal teórica, utiliza-se primeiramente o teste de Kolmogorov-Smirnov (Massey, 1951), ou teste K-S, analisando os dados coletados para cada uma das seis malhas.

Após isto, para verificar a existência de outliers, utiliza-se o teste de Grubbs (Grubbs, 1969).

Após o tratamento dos outliers, aplica-se novamente o teste de KolmogorovSmirnov para verificar se os dados restantes da amostra seguem uma distribuição normal.

Importante ressaltar que este teste pode apresentar resultados diferentes caso se utilize outros métodos ou outros níveis de significância.

Para o detalhamento de aplicação do teste, serão utilizados os dados da Malha 1. Infere-se que a amostra das incertezas dos teores de água segue uma Distribuição Normal. Como para representar uma Distribuição Normal é necessário definir sua média $\mu$ e sua variância $\sigma^{2}$, utilizou-se como estimadores os valores $\overline{\mathrm{X}}$ e $\mathrm{S}^{2}$, a média e a variância da amostra, respectivamente.

Adota-se as Hipóteses:

$\mathrm{H}_{0}$ : Dados seguem distribuição teórica Normal $\left(\overline{\mathrm{X}} ; \mathrm{S}^{2}\right)$;

$\mathrm{H}_{1}$ : Dados não seguem a distribuição teórica.

Utilizou-se o teste K-S aos níveis de significância de 1 \%. Para isto, calculase e compara-se a frequência absoluta acumulada da amostra para cada intervalo e a frequência acumulada teórica, obtendo a maior estatística D. Sendo:

$\mathrm{S}\left(x_{i}\right)$ - Frequência acumulada da amostra;

$\operatorname{Fo}\left(x_{i}\right)$ - Frequência acumulada teórica;

D - Maior diferença entre a frequência acumulada e a frequência teórica.

Após isso, observa-se a maior estatística D. Compara-se, então, com os valores $\mathrm{D}_{\max }$ da tabela Dixon, Tabela 38 , com o número de intervalos igual ao número de itens da amostra. Em alguns casos, foi necessário fazer interpolação da tabela Dixon para executar o cálculo. 
Tabela 1 - Estatísticas da amostra inicial de incerteza do teor de água para a Malha 1.

\begin{tabular}{|c|c|}
\hline Média & 0,01021 \\
\hline Desvio Padrão & 0,00520 \\
\hline Variância & 0,00003 \\
\hline
\end{tabular}

Faz-se o teste K-S adotando as seguintes hipóteses:

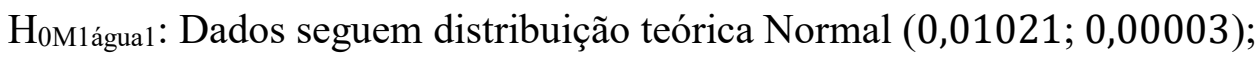

$\mathrm{H}_{1 \mathrm{Mlágual}}$ : Dados não seguem a distribuição Normal.

Tabela 2 - Dados amostrais iniciais para incerteza do teor de água da Malha 1

\begin{tabular}{|l|c|c|c|c|c|}
\hline Amostra & $\mathbf{S}(\mathbf{x i})$ & $\mathbf{S}(\mathbf{x i}-\mathbf{1})$ & $\mathbf{F o}(\mathbf{x i})$ & Fo(xi)-S(xi) & Fo(xi)-S(xi-1) \\
\hline $\mathbf{0 , 0 0 4 5 6}$ & 0,04348 & 0,00000 & 0,13863 & 0,09516 & 0,13863 \\
\hline $\mathbf{0 , 0 0 5 1 2}$ & 0,08696 & 0,04348 & 0,16380 & 0,07685 & 0,12032 \\
\hline $\mathbf{0 , 0 0 7 0 9}$ & 0,13043 & 0,08696 & 0,27426 & 0,14382 & 0,18730 \\
\hline $\mathbf{0 , 0 0 7 2 8}$ & 0,17391 & 0,13043 & 0,28647 & 0,11255 & 0,15603 \\
\hline $\mathbf{0 , 0 0 7 2 9}$ & 0,21739 & 0,17391 & 0,28697 & 0,06957 & 0,11305 \\
\hline $\mathbf{0 , 0 0 7 2 9}$ & 0,26087 & 0,21739 & 0,28706 & 0,02619 & 0,06967 \\
\hline $\mathbf{0 , 0 0 7 2 9}$ & 0,30435 & 0,26087 & 0,28710 & 0,01724 & 0,02624 \\
\hline $\mathbf{0 , 0 0 7 3 2}$ & 0,34783 & 0,30435 & 0,28937 & 0,05845 & 0,01497 \\
\hline $\mathbf{0 , 0 0 7 3 6}$ & 0,39130 & 0,34783 & 0,29167 & 0,09963 & 0,05616 \\
\hline $\mathbf{0 , 0 0 7 7 3}$ & 0,43478 & 0,39130 & 0,31671 & 0,11807 & 0,07460 \\
\hline $\mathbf{0 , 0 0 7 8 8}$ & 0,47826 & 0,43478 & 0,32722 & 0,15104 & 0,10756 \\
\hline $\mathbf{0 , 0 0 8 1 8}$ & 0,52174 & 0,47826 & 0,34833 & 0,17341 & 0,12993 \\
\hline $\mathbf{0 , 0 0 8 9 9}$ & 0,56522 & 0,52174 & 0,40694 & 0,15827 & 0,11480 \\
\hline $\mathbf{0 , 0 0 9 6 3}$ & 0,60870 & 0,56522 & 0,45559 & 0,15310 & 0,10963 \\
\hline $\mathbf{0 , 0 1 0 3 3}$ & 0,65217 & 0,60870 & 0,50878 & 0,14339 & 0,09992 \\
\hline $\mathbf{0 , 0 1 0 8 9}$ & 0,69565 & 0,65217 & 0,55192 & 0,14373 & 0,10025 \\
\hline $\mathbf{0 , 0 1 0 8 9}$ & 0,73913 & 0,69565 & 0,55192 & 0,18721 & 0,14373 \\
\hline $\mathbf{0 , 0 1 1 3 9}$ & 0,78261 & 0,73913 & 0,58955 & 0,19306 & 0,14958 \\
\hline $\mathbf{0 , 0 1 1 3 9}$ & 0,82609 & 0,78261 & 0,58955 & 0,23654 & 0,19306 \\
\hline $\mathbf{0 , 0 1 1 3 9}$ & 0,86957 & 0,82609 & 0,58981 & 0,27975 & 0,23627 \\
\hline $\mathbf{0 , 0 1 6 2 4}$ & 0,91304 & 0,86957 & 0,87685 & 0,03620 & 0,00728 \\
\hline $\mathbf{0 , 0 2 4 6 7}$ & 0,95652 & 0,91304 & 0,99727 & 0,04075 & 0,08423 \\
\hline $\mathbf{0 , 0 2 4 6 7}$ & 1,00000 & 0,95652 & 0,99727 & 0,00273 & 0,04075 \\
\hline
\end{tabular}

O maior valor de D encontrado na Tabela 2 foi 0,2798 . O valor de $D_{\max }$ na tabela de Dixon para 23 observações (foi necessário interpolar os dados) ao nível de significância de $1 \%$ foi 0,3344 . Portanto, pode-se aceitar a hipótese nula para o nível de significância de $1 \%$.

Verifica-se, então se há outliers nos valores analisados, utilizando o teste de Grubbs, calculando a estatística Z:

$$
Z=\frac{\left|x_{i}-\bar{x}\right|}{S}
$$

Sendo: 
$\mathrm{Z}$ - estatística do teste de Grubbs;

$x_{i}$ - observação da amostra;

$\bar{x}$ - média da amostra;

$S$ - desvio-padrão da amostra.

Tabela 3 - Cálculo da Estatística Z para incerteza do teor de água da Malha 1

\begin{tabular}{|c|c|}
\hline Amostra & $\mathbf{Z}$ \\
\hline $\mathbf{0 , 0 0 4 5 6}$ & 1,086 \\
\hline $\mathbf{0 , 0 0 5 1 2}$ & 0,979 \\
\hline $\mathbf{0 , 0 0 7 0 9 1}$ & 0,600 \\
\hline $\mathbf{\ldots}$ & $\ldots$ \\
\hline $\mathbf{0 , 0 1 6 2 4 3}$ & 1,159 \\
\hline $\mathbf{0 , 0 2 4 6 6 9}$ & 2,779 \\
\hline $\mathbf{0 , 0 2 4 6 6 9}$ & 2,779 \\
\hline
\end{tabular}

Optou-se por demonstrar somente os valores extremos da amostra, Tabela 3, pois estes produzem os maiores valores para estatística Z. Após isto, compara-se este valor com o valor crítico na tabela de Grubbs com $5 \%$ de significância, conforma Tabela 39.

Como o valor crítico para 23 observações ao nível de significância $1 \%$ é 2,624, considera-se que é necessário, neste caso, a retirada de outliers. Exclui-se as duas últimas observações.

Tem-se, então, uma nova estatística com os demais dados da amostra.

Tabela 4 - Estatísticas da amostra para a Malha 1 após retirada de outlier

\begin{tabular}{|c|c|}
\hline Média & 0,00884 \\
\hline Desvio Padrão & 0,00262 \\
\hline Variância & 0,00001 \\
\hline
\end{tabular}

Novo teste de hipótese posterior à retirada dos outliers:

$\mathrm{H}_{0 \mathrm{Mlágua2}}$ : Dados seguem distribuição teórica Normal (0,00884; 0,00001);

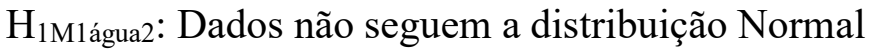

Como o maior valor de D encontrado na Tabela 5 foi 0,16948 e o valor de $\mathrm{D}_{\max }$ na tabela de Dixon para 21 observações (foi necessário interpolar os dados) ao nível de significância de $1 \%$ foi 0,3488 , pode-se aceitar a hipótese nula para o nível de significância de $1 \%$. 
Tabela 5 - Dados amostrais posteriores a retirada de outliers - incerteza do teor de água Malha 1

\begin{tabular}{|l|c|c|c|c|c|}
\hline Amostra & $\mathbf{S}(\mathbf{x i})$ & $\mathbf{S}(\mathbf{x i}-\mathbf{1})$ & $\mathbf{F o}(\mathbf{x i})$ & $\mathbf{F o}(\mathbf{x i )}-\mathbf{S}(\mathbf{x i})$ & Fo(xi)-S(xi-1) \\
\hline $\mathbf{0 , 0 0 4 5 6}$ & 0,04762 & 0,00000 & 0,05153 & 0,00391 & 0,05153 \\
\hline $\mathbf{0 , 0 0 5 1 2}$ & 0,09524 & 0,04762 & 0,07826 & 0,01698 & 0,03064 \\
\hline $\mathbf{0 , 0 0 7 0 9}$ & 0,14286 & 0,09524 & 0,25298 & 0,11012 & 0,15774 \\
\hline $\mathbf{0 , 0 0 7 2 8}$ & 0,19048 & 0,14286 & 0,27651 & 0,08603 & 0,13365 \\
\hline $\mathbf{0 , 0 0 7 2 9}$ & 0,23810 & 0,19048 & 0,27748 & 0,03939 & 0,08701 \\
\hline $\mathbf{0 , 0 0 7 2 9}$ & 0,28571 & 0,23810 & 0,27767 & 0,00805 & 0,03957 \\
\hline $\mathbf{0 , 0 0 7 2 9}$ & 0,33333 & 0,28571 & 0,27775 & 0,05558 & 0,00796 \\
\hline $\mathbf{0 , 0 0 7 3 2}$ & 0,38095 & 0,33333 & 0,28219 & 0,09876 & 0,05114 \\
\hline $\mathbf{0 , 0 0 7 3 6}$ & 0,42857 & 0,38095 & 0,28670 & 0,14187 & 0,09425 \\
\hline $\mathbf{0 , 0 0 7 7 3}$ & 0,47619 & 0,42857 & 0,33686 & 0,13933 & 0,09171 \\
\hline $\mathbf{0 , 0 0 7 8 8}$ & 0,52381 & 0,47619 & 0,35835 & 0,16546 & 0,11784 \\
\hline $\mathbf{0 , 0 0 8 1 8}$ & 0,57143 & 0,52381 & 0,40195 & 0,16948 & 0,12186 \\
\hline $\mathbf{0 , 0 0 8 9 9}$ & 0,61905 & 0,57143 & 0,52314 & 0,09591 & 0,04829 \\
\hline $\mathbf{0 , 0 0 9 6 3}$ & 0,66667 & 0,61905 & 0,61934 & 0,04733 & 0,00029 \\
\hline $\mathbf{0 , 0 1 0 3 3}$ & 0,71429 & 0,66667 & 0,71521 & 0,00093 & 0,04855 \\
\hline $\mathbf{0 , 0 1 0 8 9}$ & 0,76190 & 0,71429 & 0,78346 & 0,02155 & 0,06917 \\
\hline $\mathbf{0 , 0 1 0 8 9}$ & 0,80952 & 0,76190 & 0,78346 & 0,02607 & 0,02155 \\
\hline $\mathbf{0 , 0 1 1 3 9}$ & 0,85714 & 0,80952 & 0,83499 & 0,02215 & 0,02547 \\
\hline $\mathbf{0 , 0 1 1 3 9}$ & 0,90476 & 0,85714 & 0,83499 & 0,06977 & 0,02215 \\
\hline $\mathbf{0 , 0 1 1 3 9}$ & 0,95238 & 0,90476 & 0,83533 & 0,11705 & 0,06943 \\
\hline $\mathbf{0 , 0 1 6 2 4}$ & 1,00000 & 0,95238 & 0,99763 & 0,00237 & 0,04525 \\
\hline
\end{tabular}

Após isto, faz-se uma nova análise dos outliers. Neste caso, a maior estatística $\mathrm{Z}$ encontrada foi 2,825 , conforme Tabela 6 , superior ao valor crítico da tabela de Grubbs para 21 observações ao nível de significância de 5 \%, 2,580. Portanto, retira-se este outlier.

Tabela 6 - Segundo cálculo da Estatística Z para incerteza do teor de água da Malha 1

\begin{tabular}{|c|c|}
\hline Amostra & $\mathbf{Z}$ \\
\hline $\mathbf{0 , 0 0 4 5 6}$ & 1,630 \\
\hline $\mathbf{0 , 0 0 5 1 2}$ & 1,417 \\
\hline $\mathbf{0 , 0 0 7 0 9 1}$ & 0,665 \\
\hline $\mathbf{\ldots}$ & $\ldots$ \\
\hline $\mathbf{0 , 0 1 1 3 9 0}$ & 1,974 \\
\hline $\mathbf{0 , 0 1 1 3 9 3}$ & 2,975 \\
\hline $\mathbf{0 , 0 1 6 2 4 3}$ & 2,825 \\
\hline
\end{tabular}

Tem-se, então, uma terceira estatística com os demais dados da amostra.

Tabela 7 - Estatísticas da amostra para a Malha 1 após segunda retirada de outlier

\begin{tabular}{|c|c|}
\hline Média & 0,00847 \\
\hline Desvio Padrão & 0,00205 \\
\hline Variância & 0,00001 \\
\hline
\end{tabular}

Novo teste de hipótese posterior à segunda retirada dos outliers: 
HoMlágua3: Dados seguem distribuição teórica Normal (0,00847; 0,00001);

$\mathrm{H}_{1 \mathrm{M} 1 a ́ g u a 3}$ : Dados não seguem a distribuição Normal

Como o maior valor de D encontrado na Tabela 8 foi 0,16156 e o valor de $D_{\max }$ na tabela de Dixon para 20 observações ao nível de significância de $1 \%$ foi 0,3560, pode-se aceitar a hipótese nula para o nível de significância de $1 \%$.

Após isto, faz-se uma nova análise dos outliers. Neste caso, a maior estatística $\mathrm{Z}$ encontrada foi 1,428 , inferior ao valor crítico da tabela de Grubbs para 20 observações ao nível de significância de $5 \%, 2.580$. Portanto, não se retirou mais nenhum outlier.

Repete-se, então, a mesma sequência de cálculo para a incerteza do teor de água da Malha 2.

Tabela 8 - Dados amostrais posteriores a segunda retirada de outliers - incerteza do teor de água Malha 1

\begin{tabular}{|l|c|c|c|c|c|}
\hline Amostra & $\mathbf{S}(\mathbf{x i})$ & $\mathbf{S}(\mathbf{x i}-\mathbf{1})$ & $\mathbf{F o}(\mathbf{x i})$ & $\mathbf{F o ( x i )}-\mathbf{S}(\mathbf{x i})$ & Fo(xi)-S(xi-1) \\
\hline $\mathbf{0 , 0 0 4 5 6}$ & 0,05000 & 0,00000 & 0,02847 & 0,02153 & 0,02847 \\
\hline $\mathbf{0 , 0 0 5 1 2}$ & 0,10000 & 0,05000 & 0,05143 & 0,04857 & 0,00143 \\
\hline $\mathbf{0 , 0 0 7 0 9}$ & 0,15000 & 0,10000 & 0,25146 & 0,10146 & 0,15146 \\
\hline $\mathbf{0 , 0 0 7 2 8}$ & 0,20000 & 0,15000 & 0,28165 & 0,08165 & 0,13165 \\
\hline $\mathbf{0 , 0 0 7 2 9}$ & 0,25000 & 0,20000 & 0,28291 & 0,03291 & 0,08291 \\
\hline $\mathbf{0 , 0 0 7 2 9}$ & 0,30000 & 0,25000 & 0,28314 & 0,01686 & 0,03314 \\
\hline $\mathbf{0 , 0 0 7 2 9}$ & 0,35000 & 0,30000 & 0,28326 & 0,06674 & 0,01674 \\
\hline $\mathbf{0 , 0 0 7 3 2}$ & 0,40000 & 0,35000 & 0,28899 & 0,11101 & 0,06101 \\
\hline $\mathbf{0 , 0 0 7 3 6}$ & 0,45000 & 0,40000 & 0,29483 & 0,15517 & 0,10517 \\
\hline $\mathbf{0 , 0 0 7 7 3}$ & 0,50000 & 0,45000 & 0,36026 & 0,13974 & 0,08974 \\
\hline $\mathbf{0 , 0 0 7 8 8}$ & 0,55000 & 0,50000 & 0,38844 & 0,16156 & 0,11156 \\
\hline $\mathbf{0 , 0 0 8 1 8}$ & 0,60000 & 0,55000 & 0,44556 & 0,15444 & 0,10444 \\
\hline $\mathbf{0 , 0 0 8 9 9}$ & 0,65000 & 0,60000 & 0,60056 & 0,04944 & 0,00056 \\
\hline $\mathbf{0 , 0 0 9 6 3}$ & 0,70000 & 0,65000 & 0,71531 & 0,01531 & 0,06531 \\
\hline $\mathbf{0 , 0 1 0 3 3}$ & 0,75000 & 0,70000 & 0,81799 & 0,06799 & 0,11799 \\
\hline $\mathbf{0 , 0 1 0 8 9}$ & 0,80000 & 0,75000 & 0,88159 & 0,08159 & 0,13159 \\
\hline $\mathbf{0 , 0 1 0 8 9}$ & 0,85000 & 0,80000 & 0,88159 & 0,03159 & 0,08159 \\
\hline $\mathbf{0 , 0 1 1 3 9}$ & 0,90000 & 0,85000 & 0,92308 & 0,02308 & 0,07308 \\
\hline $\mathbf{0 , 0 1 1 3 9}$ & 0,95000 & 0,90000 & 0,92308 & 0,02692 & 0,02308 \\
\hline $\mathbf{0 , 0 1 1 3 9}$ & 1,00000 & 0,95000 & 0,92333 & 0,07667 & 0,02667 \\
\hline
\end{tabular}

- Malha 2 - Incerteza do teor de água:

Tabela 9 - Estatísticas iniciais da amostra para a Malha 2

\begin{tabular}{|c|c|}
\hline Média & 0,01187 \\
\hline Desvio Padrão & 0,00817 \\
\hline Variância & 0,00007 \\
\hline
\end{tabular}


Hipóteses do teste K-S:

Hom2águal: Dados seguem distribuição teórica Normal (0,01187; 0,00007);

$\mathrm{H}_{1 \mathrm{M} 2 a ́ g u a 1}$ : Dados não seguem a distribuição Normal.

Tabela 10 - Dados amostrais iniciais - incerteza do teor de água Malha 2

\begin{tabular}{|c|c|c|c|c|c|}
\hline Amostra & S(xi) & $\mathbf{S}(\mathbf{x i}-\mathbf{1})$ & Fo(xi) & Fo(xi)-S(xi) & Fo(xi)-S(xi-1) \\
\hline $\mathbf{0 , 0 0 5 1 2 4}$ & 0,03846 & 0,00000 & 0,20468 & 0,16622 & 0,20468 \\
\hline $\mathbf{0 , 0 0 6 6 4 8}$ & 0,07692 & 0,03846 & 0,26156 & 0,18464 & 0,22310 \\
\hline $\mathbf{0 , 0 0 6 9 9 8}$ & 0,11538 & 0,07692 & 0,27567 & 0,16028 & 0,19874 \\
\hline $\mathbf{0 , 0 0 7 0 2 9}$ & 0,15385 & 0,11538 & 0,27693 & 0,12309 & 0,16155 \\
\hline $\mathbf{0 , 0 0 7 0 2 9}$ & 0,19231 & 0,15385 & 0,27693 & 0,08463 & 0,12309 \\
\hline $\mathbf{0 , 0 0 7 0 3 7}$ & 0,23077 & 0,19231 & 0,27725 & 0,04648 & 0,08494 \\
\hline $\mathbf{0 , 0 0 7 0 4 5}$ & 0,26923 & 0,23077 & 0,27760 & 0,00837 & 0,04683 \\
\hline $\mathbf{0 , 0 0 7 0 6 7}$ & 0,30769 & 0,26923 & 0,27851 & 0,02918 & 0,00928 \\
\hline $\mathbf{0 , 0 0 7 0 8 7}$ & 0,34615 & 0,30769 & 0,27930 & 0,06685 & 0,02839 \\
\hline $\mathbf{0 , 0 0 7 0 9 6}$ & 0,38462 & 0,34615 & 0,27970 & 0,10492 & 0,06646 \\
\hline $\mathbf{0 , 0 0 7 2 1 7}$ & 0,42308 & 0,38462 & 0,28470 & 0,13837 & 0,09991 \\
\hline $\mathbf{0 , 0 0 7 2 4 9}$ & 0,46154 & 0,42308 & 0,28604 & 0,17550 & 0,13704 \\
\hline $\mathbf{0 , 0 0 7 2 9 9}$ & 0,50000 & 0,46154 & 0,28810 & 0,21190 & 0,17344 \\
\hline $\mathbf{0 , 0 0 7 3 3}$ & 0,53846 & 0,50000 & 0,28940 & 0,24906 & 0,21060 \\
\hline $\mathbf{0 , 0 0 7 9 2 6}$ & 0,57692 & 0,53846 & 0,31482 & 0,26210 & 0,22364 \\
\hline $\mathbf{0 , 0 0 7 9 8 3}$ & 0,61538 & 0,57692 & 0,31730 & 0,29808 & 0,25962 \\
\hline $\mathbf{0 , 0 0 8 6 6 3}$ & 0,65385 & 0,61538 & 0,34751 & 0,30633 & 0,26787 \\
\hline $\mathbf{0 , 0 1 3 3 4 7}$ & 0,69231 & 0,65385 & 0,57180 & 0,12051 & 0,08205 \\
\hline $\mathbf{0 , 0 1 3 7 8 4}$ & 0,73077 & 0,69231 & 0,59267 & 0,13810 & 0,09964 \\
\hline $\mathbf{0 , 0 1 3 7 8 7}$ & 0,76923 & 0,73077 & 0,59280 & 0,17643 & 0,13797 \\
\hline $\mathbf{0 , 0 1 3 8 2 1}$ & 0,80769 & 0,76923 & 0,59441 & 0,21328 & 0,17482 \\
\hline $\mathbf{0 , 0 1 7 3 8 5}$ & 0,84615 & 0,80769 & 0,75012 & 0,09604 & 0,05758 \\
\hline $\mathbf{0 , 0 2 4 6 9 2}$ & 0,88462 & 0,84615 & 0,94166 & 0,05704 & 0,09551 \\
\hline $\mathbf{0 , 0 2 4 6 9 2}$ & 0,92308 & 0,88462 & 0,94166 & 0,01858 & 0,05704 \\
\hline $\mathbf{0 , 0 2 7 4 6 6}$ & 0,96154 & 0,92308 & 0,97181 & 0,01028 & 0,04874 \\
\hline $\mathbf{0 , 0 3 7 7 6 4}$ & 1,00000 & 0,96154 & 0,99923 & 0,00077 & 0,03769 \\
\hline & & & & & \\
\hline
\end{tabular}

O maior valor de $\mathrm{D}$ encontrado na Tabela 10 foi 0,3063 . $O$ valor de $D_{\max }$ na tabela de Dixon para 26 observações (foi necessário interpolar os dados) ao nível de significância de $1 \%$ foi 0,3128 . Portanto, pode-se aceitar a hipótese nula para o nível de significância de $1 \%$. 
Tabela 11 - Cálculo da Estatística Z para incerteza do teor de água da Malha 2

\begin{tabular}{|c|c|}
\hline Amostra & $\mathbf{Z}$ \\
\hline $\mathbf{0 , 0 0 5 1 2 4}$ & 0,825 \\
\hline $\mathbf{0 , 0 0 6 6 4 8}$ & 0,639 \\
\hline $\mathbf{0 , 0 0 6 9 9 8}$ & 0,596 \\
\hline $\mathbf{\ldots}$ & $\ldots$ \\
\hline $\mathbf{0 , 0 2 4 6 9 2}$ & 1,569 \\
\hline $\mathbf{0 , 0 2 7 4 6 6}$ & 1,908 \\
\hline $\mathbf{0 , 0 3 7 7 6 4}$ & 3,168 \\
\hline
\end{tabular}

Neste caso, como o valor crítico para 26 observações ao nível de significância $5 \%$ é 2,6841, exclui-se a última observação.

Tem-se, então, uma nova estatística com os demais dados da amostra.

Tabela 12 - Estatísticas da amostra para a Malha 2 após retirada de outlier

\begin{tabular}{|c|c|}
\hline Média & 0,01083 \\
\hline Desvio Padrão & 0,00637 \\
\hline Variância & 0,00004 \\
\hline
\end{tabular}

Novo teste de hipótese posterior à retirada dos outliers:

$\mathrm{H}_{0 \mathrm{M} 2 a ́ g u a 2}$ : Dados seguem distribuição teórica Normal (0,01083; 0,00004);

$\mathrm{H}_{1 \mathrm{M} 2 a ́ g u a 2}$ : Dados não seguem a distribuição Normal

Como o maior valor de D encontrado na Tabela 13 foi 0,3133 e o valor de $\mathrm{D}_{\max }$ na tabela de Dixon para 25 observações ao nível de significância de $1 \%$ foi 0,32, pode-se aceitar a hipótese nula para o nível de significância de $1 \%$.

Após isto, faz-se uma nova análise dos outliers. Neste caso, a maior estatística $\mathrm{Z}$ encontrada foi 2,61231, inferior ao valor crítico da tabela de Grubbs para 25 observações ao nível de significância de 5 \%, 2,663. Portanto, não se retirou mais nenhum outlier.

Os demais dados foram tratados com a mesma metodologia definida acima. Este tratamento será apresentado de forma simplificada a partir deste ponto. 
Tabela 13 - Dados amostrais posteriores a retirada de outliers - incerteza do teor de água Malha 2

\begin{tabular}{|c|c|c|c|c|c|}
\hline Amostra & S(xi) & S(xi-1) & Fo(xi) & Fo(xi)-S(xi) & Fo(xi)-S(xi-1) \\
\hline $\mathbf{0 , 0 0 5 1 2 4}$ & 0,04000 & 0,00000 & 0,18500 & 0,14500 & 0,18500 \\
\hline $\mathbf{0 , 0 0 6 6 4 8}$ & 0,08000 & 0,04000 & 0,25557 & 0,17557 & 0,21557 \\
\hline $\mathbf{0 , 0 0 6 9 9 8}$ & 0,12000 & 0,08000 & 0,27354 & 0,15354 & 0,19354 \\
\hline $\mathbf{0 , 0 0 7 0 2 9}$ & 0,16000 & 0,12000 & 0,27516 & 0,11516 & 0,15516 \\
\hline $\mathbf{0 , 0 0 7 0 2 9}$ & 0,20000 & 0,16000 & 0,27516 & 0,07516 & 0,11516 \\
\hline $\mathbf{0 , 0 0 7 0 3 7}$ & 0,24000 & 0,20000 & 0,27557 & 0,03557 & 0,07557 \\
\hline $\mathbf{0 , 0 0 7 0 4 5}$ & 0,28000 & 0,24000 & 0,27601 & 0,00399 & 0,03601 \\
\hline $\mathbf{0 , 0 0 7 0 6 7}$ & 0,32000 & 0,28000 & 0,27718 & 0,04282 & 0,00282 \\
\hline $\mathbf{0 , 0 0 7 0 8 7}$ & 0,36000 & 0,32000 & 0,27819 & 0,08181 & 0,04181 \\
\hline $\mathbf{0 , 0 0 7 0 9 6}$ & 0,40000 & 0,36000 & 0,27870 & 0,12130 & 0,08130 \\
\hline $\mathbf{0 , 0 0 7 2 1 7}$ & 0,44000 & 0,40000 & 0,28512 & 0,15488 & 0,11488 \\
\hline $\mathbf{0 , 0 0 7 2 4 9}$ & 0,48000 & 0,44000 & 0,28684 & 0,19316 & 0,15316 \\
\hline $\mathbf{0 , 0 0 7 2 9 9}$ & 0,52000 & 0,48000 & 0,28949 & 0,23051 & 0,19051 \\
\hline $\mathbf{0 , 0 0 7 3 3}$ & 0,56000 & 0,52000 & 0,29117 & 0,26883 & 0,22883 \\
\hline $\mathbf{0 , 0 0 7 9 2 6}$ & 0,60000 & 0,56000 & 0,32405 & 0,27595 & 0,23595 \\
\hline $\mathbf{0 , 0 0 7 9 8 3}$ & 0,64000 & 0,60000 & 0,32728 & 0,31272 & 0,27272 \\
\hline $\mathbf{0 , 0 0 8 6 6 3}$ & 0,68000 & 0,64000 & 0,36670 & 0,31330 & 0,27330 \\
\hline $\mathbf{0 , 0 1 3 3 4 7}$ & 0,72000 & 0,68000 & 0,65357 & 0,06643 & 0,02643 \\
\hline $\mathbf{0 , 0 1 3 7 8 4}$ & 0,76000 & 0,72000 & 0,67854 & 0,08146 & 0,04146 \\
\hline $\mathbf{0 , 0 1 3 7 8 7}$ & 0,80000 & 0,76000 & 0,67869 & 0,12131 & 0,08131 \\
\hline $\mathbf{0 , 0 1 3 8 2 1}$ & 0,84000 & 0,80000 & 0,68060 & 0,15940 & 0,11940 \\
\hline $\mathbf{0 , 0 1 7 3 8 5}$ & 0,88000 & 0,84000 & 0,84827 & 0,03173 & 0,00827 \\
\hline $\mathbf{0 , 0 2 4 6 9 2}$ & 0,92000 & 0,88000 & 0,98525 & 0,06525 & 0,10525 \\
\hline $\mathbf{0 , 0 2 4 6 9 2}$ & 0,96000 & 0,92000 & 0,98525 & 0,02525 & 0,06525 \\
\hline $\mathbf{0 , 0 2 7 4 6 6}$ & 1,00000 & 0,96000 & 0,99550 & 0,00450 & 0,03550 \\
\hline
\end{tabular}

- Malha 3 - Incerteza do teor de água:

Tabela 14 - Amostra inicial para incerteza do teor de água - Malha 3

\begin{tabular}{|l|l|l|l|l|}
\hline \multicolumn{5}{|c|}{ Amostra } \\
\hline 0,005124 & 0,007034 & 0,007295 & 0,010929 & 0,020381 \\
\hline 0,006648 & 0,007043 & 0,007303 & 0,013821 & 0,024692 \\
\hline 0,006954 & 0,007051 & 0,007320 & 0,014265 & 0,024692 \\
\hline 0,006970 & 0,007066 & 0,007607 & 0,014997 & 0,037764 \\
\hline 0,007024 & 0,007280 & 0,008007 & 0,016820 & \\
\hline
\end{tabular}

Tabela 15 - Estatísticas iniciais - incerteza do teor de água para a Malha 3

\begin{tabular}{|c|c|}
\hline Média & 0,01184 \\
\hline Desvio Padrão & 0,00800 \\
\hline Variância & 0,00006 \\
\hline
\end{tabular}


Teste K-S inicial:

HoM3águal: Dados seguem distribuição teórica Normal (0,01184; 0,00006);

$\mathrm{H}_{1 \mathrm{M} 3 a ́ g u a 1}$ : Dados não seguem a distribuição Normal.

O maior valor de D encontrado para esta amostra foi 0,309042. O valor de $\mathrm{D}_{\max }$ na tabela de Dixon para 24 observações (foi necessário interpolar os dados) ao nível de significância de $1 \%$ foi 0,3272 . Portanto, pode-se aceitar a hipótese nula para o nível de significância de $1 \%$.

Ao calcular a estatística $\mathrm{Z}$ para os dados da amostra, decidiu-se por excluir a observação 0,037764 , pois unicamente o valor de Z para esta $(3,245)$ ultrapassa o valor máximo da tabela de Grubbs para 24 observações ao nível de significância de $5 \%, 2,644$.

Tabela 16 - Estatísticas da amostra para a Malha 3 após retirada de outlier

\begin{tabular}{|c|c|}
\hline Média & 0,01071 \\
\hline Desvio Padrão & 0,00591 \\
\hline Variância & 0,00003 \\
\hline
\end{tabular}

Novo Teste K-S:

HoM3água2: Dados seguem distribuição teórica Normal (0,01071; 0,00003);

$\mathrm{H}_{1 \mathrm{M} 3 a ́ g u a 2}$ Dados não seguem a distribuição Normal.

O maior valor de $\mathrm{D}$ encontrado para esta amostra foi 0,3284 . O valor de $D_{\max }$ na tabela de Dixon para 23 observações (foi necessário interpolar os dados) ao nível de significância de $1 \%$ foi 0,3344 . Portanto, pode-se aceitar a hipótese nula para o nível de significância de $1 \%$.

Após isto, repete-se o teste de Grubbs, porém nenhum valor de estatística Z ultrapassou o valor tabelado para $5 \%$ de significância e 23 observações.

- Malha 4 - Incerteza do teor de água:

Tabela 17 - Amostra inicial para incerteza do teor de água - Malha 4

\begin{tabular}{|l|l|l|l|l|}
\hline \multicolumn{5}{|c|}{ Amostra } \\
\hline 0,004558 & 0,005030 & 0,007442 & 0,008103 & 0,012144 \\
\hline 0,004558 & 0,005030 & 0,007585 & 0,008115 & 0,012144 \\
\hline 0,005010 & 0,005127 & 0,007830 & 0,009270 & 0,012950 \\
\hline 0,005010 & 0,005127 & 0,008101 & 0,012073 & 0,012950 \\
\hline 0,005030 & 0,006185 & 0,008103 & 0,012144 & 0,020423 \\
\hline
\end{tabular}


Tabela 18 - Estatísticas iniciais - incerteza do teor de água para a Malha 4

\begin{tabular}{|c|c|}
\hline Média & 0,00840 \\
\hline Desvio Padrão & 0,00386 \\
\hline Variância & 0,00001 \\
\hline
\end{tabular}

Teste K-S inicial:

Hom4águal: Dados seguem distribuição teórica Normal (0,00840; 0,00001);

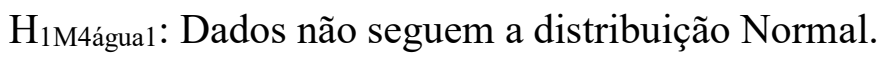

O maior valor de D encontrado para os dados desta amostra foi 0,2096. O valor de $D_{\max }$ na tabela de Dixon para 25 observações ao nível de significância de $1 \%$ foi 0,3200. Portanto, pode-se aceitar a hipótese nula para o nível de significância de $1 \%$.

Ao calcular a estatística $Z$ para os dados da amostra, decidiu-se por excluir a observação 0,020423 , pois unicamente o valor de Z para esta $(3,115)$ ultrapassa o valor máximo da tabela de Grubbs para 25 observações ao nível de significância de $5 \%, 2,663$.

Tabela 19 - Estatísticas da amostra para a Malha 4 após retirada de outlier

\begin{tabular}{|c|c|}
\hline Média & 0,00790 \\
\hline Desvio Padrão & 0,00300 \\
\hline Variância & 0,00001 \\
\hline
\end{tabular}

Novo Teste K-S:

$\mathrm{H}_{0 \mathrm{M} 4 a ́ g u a 2}$ : Dados seguem distribuição teórica Normal (0,00790; 0,00001);

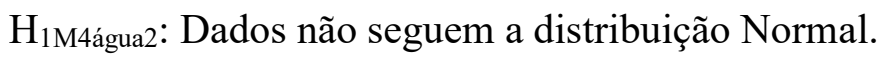

O maior valor de $\mathrm{D}$ encontrado para esta amostra foi 0,1975 . O valor de $\mathrm{D}_{\max }$ na tabela de Dixon para 24 observações (foi necessário interpolar os dados) ao nível de significância de $1 \%$ foi 0,3272 . Portanto, pode-se aceitar a hipótese nula para o nível de significância de $1 \%$.

Após isto, repete-se o teste de Grubbs, porém nenhum valor de estatística $Z$ ultrapassou o valor tabelado para $5 \%$ de significância e 24 observações. 
- Malha 5 - Incerteza do teor de água:

Tabela 20 - Amostra inicial para incerteza do teor de água - Malha 5

\begin{tabular}{|l|l|l|l|l|}
\hline \multicolumn{5}{|c|}{ Amostra } \\
\hline 0,001947 & 0,004789 & 0,007568 & 0,010500 & 0,020390 \\
\hline 0,004692 & 0,005110 & 0,008100 & 0,012150 & \\
\hline 0,004692 & 0,005111 & 0,008128 & 0,012150 & \\
\hline 0,004789 & 0,005215 & 0,008158 & 0,012150 & \\
\hline 0,004789 & 0,007455 & 0,009452 & 0,012910 & \\
\hline
\end{tabular}

Tabela 21 - Estatísticas iniciais - incerteza do teor de água para a Malha 5

\begin{tabular}{|c|c|}
\hline Média & 0,00811 \\
\hline Desvio Padrão & 0,00419 \\
\hline Variância & 0,00002 \\
\hline
\end{tabular}

Teste K-S inicial:

H0M5águal: Dados seguem distribuição teórica Normal (0,00811; 0,00002);

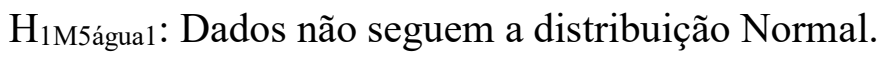

O maior valor de $\mathrm{D}$ encontrado para esta amostra foi 0,1834 . O valor de $\mathrm{D}_{\max }$ na tabela de Dixon para 21 observações (foi necessário interpolar os dados) ao nível de significância de $1 \%$ foi 0,3488 . Portanto, pode-se aceitar a hipótese nula para o nível de significância de $1 \%$.

Ao calcular a estatística $\mathrm{Z}$ para os dados da amostra, decidiu-se por excluir a observação 0,020390 , pois unicamente o valor de Z para esta $(2,928)$ ultrapassa o valor máximo da tabela de Grubbs para 21 observações ao nível de significância de $5 \%, 2,580$.

Tabela 22 - Estatísticas da amostra para a Malha 5 após retirada de outlier

\begin{tabular}{|c|c|}
\hline Média & 0,00749 \\
\hline Desvio Padrão & 0,00319 \\
\hline Variância & 0,00001 \\
\hline
\end{tabular}

Novo Teste K-S:

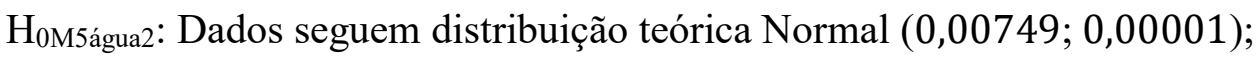

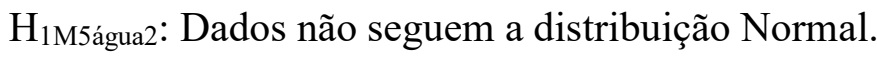


O maior valor de $\mathrm{D}$ encontrado para esta amostra foi 0,2124 . $\mathrm{O}$ valor de $\mathrm{D}_{\max }$ na tabela de Dixon para 20 observações ao nível de significância de $1 \%$ foi 0,3560 . Portanto, pode-se aceitar a hipótese nula para o nível de significância de $1 \%$.

Após isto, repete-se o teste de Grubbs, porém nenhum valor de estatística Z ultrapassou o valor tabelado para $5 \%$ de significância e 20 observações.

\section{- Malha 6 - Incerteza do teor de água:}

Tabela 23 - Amostra inicial para incerteza do teor de água - Malha 6

\begin{tabular}{|l|l|l|l|l|}
\hline \multicolumn{5}{|c|}{ Amostras } \\
\hline 0,00195 & 0,00535 & 0,00543 & 0,01057 & 0,01290 \\
\hline 0,00326 & 0,00535 & 0,00808 & 0,01286 & 0,02075 \\
\hline 0,00531 & 0,00541 & 0,00821 & 0,01286 & \\
\hline 0,00535 & 0,00541 & 0,00876 & 0,01290 & \\
\hline 0,00535 & 0,00541 & 0,00986 & 0,01290 & \\
\hline
\end{tabular}

Tabela 24 - Estatísticas iniciais - incerteza do teor de água para a Malha 6

\begin{tabular}{|c|c|}
\hline Média & 0,00837 \\
\hline Desvio Padrão & 0,00442 \\
\hline Variância & 0,00002 \\
\hline
\end{tabular}

Teste K-S inicial:

Hom6águal: Dados seguem distribuição teórica Normal (0,00837; 0,00002);

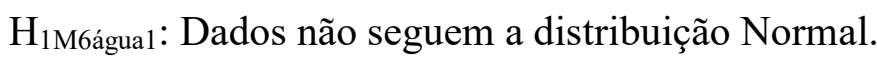

O maior valor de D encontrado na amostra foi 0,2472 . O valor de $D_{\max }$ na tabela de Dixon para 22 observações (foi necessário interpolar os dados) ao nível de significância de $1 \%$ foi 0,3416 . Portanto, pode-se aceitar a hipótese nula para o nível de significância de $1 \%$.

Ao calcular a estatística $\mathrm{Z}$ para os dados da amostra, decidiu-se por excluir a observação 0,02075 , pois unicamente o valor de Z para esta $(2,799)$ ultrapassa o valor máximo da tabela de Grubbs para 22 observações ao nível de significância de $5 \%, 2,603$. 
Tabela 25 - Estatísticas da amostra para a Malha 6 após retirada de outlier

\begin{tabular}{|c|c|}
\hline Média & 0,00779 \\
\hline Desvio Padrão & 0,00354 \\
\hline Variância & 0,00001 \\
\hline
\end{tabular}

Novo Teste K-S:

$\mathrm{H}_{0 \mathrm{M} 6 a ́ g u a 2}$ : Dados seguem distribuição teórica Normal (0,00779; 0,00001);

H1M6água2: Dados não seguem a distribuição Normal.

O maior valor de D encontrado para esta amostra foi 0,2710 . O valor de Dmax na tabela de Dixon para 21 observações ao nível de significância de $1 \%$ foi 0,3488. Portanto, pode-se aceitar a hipótese nula para o nível de significância de $1 \%$.

Após isto, repete-se o teste de Grubbs, porém nenhum valor de estatística Z ultrapassou o valor tabelado para $5 \%$ de significância e 21 observações.

- Malha 1 - Incerteza de MF:

Tabela 26 - Amostra inicial para incerteza de MF - Malha 1

\begin{tabular}{|l|l|l|l|l|}
\hline \multicolumn{5}{|c|}{ Amostras } \\
\hline 0,00067 & 0,00180 & 0,00531 & 0,00653 & 0,01670 \\
\hline 0,00069 & 0,00236 & 0,00548 & 0,00660 & 0,01798 \\
\hline 0,00069 & 0,00236 & 0,00614 & 0,00878 & 0,01799 \\
\hline 0,00069 & 0,00240 & 0,00615 & 0,01010 & \\
\hline 0,00133 & 0,00253 & 0,00622 & 0,01010 & \\
\hline
\end{tabular}

Tabela 27 - Estatísticas iniciais - incerteza de MF para a Malha 1

\begin{tabular}{|c|c|}
\hline Média & 0,00607 \\
\hline Desvio Padrão & 0,00543 \\
\hline Variância & 0,00003 \\
\hline
\end{tabular}

Teste K-S inicial:

$\mathrm{H}_{0 \mathrm{M} 1 \mathrm{MF} 1}$ : Dados seguem distribuição teórica Normal $(0,00607 ; 0,00003)$;

H1M1MF1: Dados não seguem a distribuição Normal.

O maior valor de $\mathrm{D}$ encontrado na amostra foi 0,2005 . O valor de $D_{\max }$ na tabela de Dixon para 23 observações (foi necessário interpolar os dados) ao nível de significância de $1 \%$ foi 0,3344 . Portanto, pode-se aceitar a hipótese nula para o nível de significância de $1 \%$. 
Ao calcular a estatística $Z$ para os dados da amostra, decidiu-se por não excluir nenhuma observação, pois o valor da mesma para nenhum dos dados ultrapassa o valor máximo da tabela de Grubbs para 23 observações ao nível de significância de $5 \%, 2,623$. Portanto, para este caso, valem as estatísticas da Tabela 26 e o primeiro teste de hipótese feito.

- Malha 2 - Incerteza de MF:

Tabela 28 - Amostra inicial para incerteza de MF - Malha 2

\begin{tabular}{|c|c|c|c|c|}
\hline \multicolumn{5}{|c|}{ Amostra } \\
\hline 0,001311 & 0,004666 & 0,007133 & 0,008632 & 0,01312 \\
\hline 0,001737 & 0,00593 & 0,007548 & 0,008901 & 0,01414 \\
\hline 0,001737 & 0,006864 & 0,007666 & 0,008901 & 0,0167 \\
\hline 0,00329 & 0,007016 & 0,007712 & 0,010868 & 0,01951 \\
\hline 0,004604 & 0,007016 & 0,007954 & 0,012046 & 0,01951 \\
\hline & & & & 0,02337 \\
\hline
\end{tabular}

Tabela 29 - Estatísticas iniciais - incerteza de MF para a Malha 2

\begin{tabular}{|c|c|}
\hline Média & 0,00915 \\
\hline Desvio Padrão & 0,00569 \\
\hline Variância & 0,00003 \\
\hline
\end{tabular}

Teste K-S inicial:

H0M2MF1: Dados seguem distribuição teórica Normal (0,00915; 0,00003);

H1M2MF1: Dados não seguem a distribuição Normal.

O maior valor de $\mathrm{D}$ encontrado na amostra foi 0,2097 . O valor de $\mathrm{D}_{\max }$ na tabela de Dixon para 26 observações (foi necessário interpolar os dados) ao nível de significância de $1 \%$ foi 0,3128 . Portanto, pode-se aceitar a hipótese nula para o nível de significância de $1 \%$.

Ao calcular a estatística $\mathrm{Z}$ para os dados da amostra, decidiu-se por não excluir nenhuma observação, pois o valor da mesma para nenhum dos dados ultrapassa o valor máximo da tabela de Grubbs para 26 observações ao nível de significância de $5 \%, 2,681$. Portanto, para este caso, valem as estatísticas da Tabela 29 e o primeiro teste de hipótese feito. 
- Malha 3 - Incerteza de MF:

Tabela 30 - Amostra inicial para incerteza de MF - Malha 3

\begin{tabular}{|c|c|c|c|c|}
\hline \multicolumn{5}{|c|}{ Amostra } \\
\hline 0,00129 & 0,004683 & 0,007954 & 0,009585 & 0,0166 \\
\hline 0,00129 & 0,005155 & 0,008632 & 0,010911 & 0,018299 \\
\hline 0,001737 & 0,005958 & 0,008793 & 0,011261 & 0,020289 \\
\hline 0,002247 & 0,007457 & 0,008901 & 0,013122 & 0,021943 \\
\hline 0,002356 & 0,007548 & 0,008901 & 0,01403 & \\
\hline
\end{tabular}

Tabela 31 - Estatísticas iniciais - incerteza de MF para a Malha 3

\begin{tabular}{|c|c|}
\hline Média & 0,00912 \\
\hline Desvio Padrão & 0,00590 \\
\hline Variância & 0,00003 \\
\hline
\end{tabular}

Teste K-S inicial:

HoM2MF1: Dados seguem distribuição teórica Normal (0,00912; 0,00003);

$\mathrm{H}_{1 \mathrm{M} 2 \mathrm{MF1}}$ : Dados não seguem a distribuição Normal.

O maior valor de D encontrado na amostra foi 0,1400 . O valor de $D_{\max }$ na tabela de Dixon para 24 observações (foi necessário interpolar os dados) ao nível de significância de $1 \%$ foi 0,3272 . Portanto, pode-se aceitar a hipótese nula para o nível de significância de $1 \%$.

Ao calcular a estatística $Z$ para os dados da amostra, decidiu-se por não excluir nenhuma observação, pois o valor da mesma para nenhum dos dados ultrapassa o valor máximo da tabela de Grubbs para 24 observações ao nível de significância de 5\%,2,644. Portanto, para este caso, valem as estatísticas da Tabela 31 e o primeiro teste de hipótese feito.

- Malha 4 - Incerteza de MF:

Tabela 32 - Amostra inicial para incerteza de MF - Malha 4

\begin{tabular}{|l|l|l|l|l|}
\hline \multicolumn{5}{|c|}{ Amostra } \\
\hline 0,00556 & 0,00569 & 0,00650 & 0,01019 & 0,01311 \\
\hline 0,00556 & 0,00569 & 0,00653 & 0,01043 & 0,01311 \\
\hline 0,00558 & 0,00605 & 0,00742 & 0,01275 & 0,01311 \\
\hline 0,00558 & 0,00605 & 0,00826 & 0,01282 & 0,01502 \\
\hline 0,00569 & 0,00650 & 0,00886 & 0,01284 & 0,01802 \\
\hline
\end{tabular}


Tabela 33 - Estatísticas iniciais - incerteza de MF para a Malha 4

\begin{tabular}{|c|c|}
\hline Média & 0,00908 \\
\hline Desvio Padrão & 0,00373 \\
\hline Variância & 0,00001 \\
\hline
\end{tabular}

Teste K-S inicial:

$\mathrm{H}_{0 \mathrm{M} 4 \mathrm{MF} 1}$ : Dados seguem distribuição teórica Normal (0,00908; 0,00001);

$\mathrm{H}_{1 \mathrm{M} 4 \mathrm{MF} 1}$ : Dados não seguem a distribuição Normal.

O maior valor de $\mathrm{D}$ encontrado na amostra foi 0,2322 . $O$ valor de $D_{\max }$ na tabela de Dixon para 25 observações ao nível de significância de $1 \%$ foi 0,3200 . Portanto, pode-se aceitar a hipótese nula para o nível de significância de $1 \%$.

Ao calcular a estatística $Z$ para os dados da amostra, decidiu-se por não excluir nenhuma observação, pois o valor da mesma para nenhum dos dados ultrapassa o valor máximo da tabela de Grubbs para 25 observações ao nível de significância de $5 \%$, 2,663. Portanto, para este caso, valem as estatísticas da Tabela 33 e o primeiro teste de hipótese feito.

- Malha 5 - Incerteza de MF:

Tabela 34 - Amostra inicial para incerteza de MF - Malha 5

\begin{tabular}{|l|l|l|l|l|}
\hline \multicolumn{5}{|c|}{ Amostra } \\
\hline 0,00211 & 0,00233 & 0,00805 & 0,01316 & 0,01663 \\
\hline 0,00231 & 0,00295 & 0,00870 & 0,01316 & \\
\hline 0,00231 & 0,00295 & 0,00929 & 0,01316 & \\
\hline 0,00233 & 0,00423 & 0,01043 & 0,01493 & \\
\hline 0,00233 & 0,00432 & 0,01265 & 0,01652 & \\
\hline
\end{tabular}

Tabela 35 - Estatísticas iniciais - incerteza de MF para a Malha 5

\begin{tabular}{|c|c|}
\hline Média & 0,00785 \\
\hline Desvio Padrão & 0,00538 \\
\hline Variância & 0,00003 \\
\hline
\end{tabular}

Teste K-S inicial:

$\mathrm{H}_{0 \mathrm{M} 5 \mathrm{MF} 1}$ : Dados seguem distribuição teórica Normal (0,00758; 0,00003);

$\mathrm{H}_{1 \mathrm{M} 5 \mathrm{MF} 1}$ : Dados não seguem a distribuição Normal. 
$\mathrm{O}$ maior valor de $\mathrm{D}$ encontrado na amostra foi 0,2306 . $\mathrm{O}$ valor de $\mathrm{D}_{\max }$ na tabela de Dixon para 21 observações (foi necessário interpolar os dados) ao nível de significância de $1 \%$ foi 0,3488 . Portanto, pode-se aceitar a hipótese nula para o nível de significância de $1 \%$.

Ao calcular a estatística $\mathrm{Z}$ para os dados da amostra, decidiu-se por não excluir nenhuma observação, pois o valor da mesma para nenhum dos dados ultrapassa o valor máximo da tabela de Grubbs para 21 observações ao nível de significância de $5 \%, 2,580$. Portanto, para este caso, valem as estatísticas da Tabela 35 e o primeiro teste de hipótese feito.

\section{- Malha 6 - incerteza de MF:}

Tabela 36 - Amostra inicial para incerteza de MF - Malha 6

\begin{tabular}{|l|l|l|l|l|}
\hline \multicolumn{5}{|c|}{ Amostra } \\
\hline 0,00208 & 0,00291 & 0,00400 & 0,01256 & 0,01471 \\
\hline 0,00216 & 0,00291 & 0,00417 & 0,01267 & 0,01651 \\
\hline 0,00216 & 0,00291 & 0,00499 & 0,01267 & \\
\hline 0,00268 & 0,00291 & 0,00513 & 0,01267 & \\
\hline 0,00290 & 0,00307 & 0,00513 & 0,01471 & \\
\hline
\end{tabular}

Tabela 37 - Estatísticas iniciais - incerteza de MF para a Malha 6

\begin{tabular}{|c|c|}
\hline Média & 0,00667 \\
\hline Desvio Padrão & 0,00512 \\
\hline Variância & 0,00003 \\
\hline
\end{tabular}

Teste K-S inicial:

H0M4MF1: Dados seguem distribuição teórica Normal $(0,00667 ; 0,00003)$;

$\mathrm{H}_{1 \mathrm{M} 4 \mathrm{MF1}}$ : Dados não seguem a distribuição Normal.

O maior valor de D encontrado na amostra foi 0,2992 . O valor de $D_{\max }$ na tabela de Dixon para 22 observações (foi necessário interpolar os dados) ao nível de significância de $1 \%$ foi 0,3416 . Portanto, pode-se aceitar a hipótese nula para o nível de significância de $1 \%$.

Ao calcular a estatística $Z$ para os dados da amostra, decidiu-se por não excluir nenhuma observação, pois o valor da mesma para nenhum dos dados ultrapassa o valor máximo da tabela de Grubbs para 22 observações ao nível de 
significância de $5 \%, 2,603$. Portanto, para este caso, valem as estatísticas da Tabela 37 e o primeiro teste de hipótese feito.

Tabela 38 - Valores críticos de D - Tabela adaptada de Massey, 1951.

\begin{tabular}{|c|c|}
\hline $\begin{array}{c}\text { Tamanho da } \\
\text { amostra } \\
\text { n }\end{array}$ & $D_{0,01}$ \\
\hline 1 & 0,995 \\
\hline 2 & 0,929 \\
\hline 3 & 0,828 \\
\hline 4 & 0,733 \\
\hline 5 & 0,669 \\
\hline 6 & 0,618 \\
\hline 7 & 0,577 \\
\hline 8 & 0,543 \\
\hline 9 & 0,514 \\
\hline 10 & 0,490 \\
\hline 11 & 0,468 \\
\hline 12 & 0,450 \\
\hline 13 & 0,433 \\
\hline 14 & 0,418 \\
\hline 15 & 0,404 \\
\hline 16 & 0,392 \\
\hline 17 & 0,381 \\
\hline 18 & 0,371 \\
\hline 19 & 0,363 \\
\hline 20 & 0,356 \\
\hline 25 & 0,32 \\
\hline 30 & 0,29 \\
\hline
\end{tabular}


Tabela 39 - Estatística Z - Tabela adaptada de Grubbs, 1969.

\begin{tabular}{|c|c|}
\hline $\begin{array}{l}\text { Número de } \\
\text { Observações }\end{array}$ & $\begin{array}{c}\text { Valor Crítico para } \\
5 \% \text { de } \\
\text { significância }\end{array}$ \\
\hline 10 & 2,176 \\
\hline 11 & 2,234 \\
\hline 12 & 2,285 \\
\hline 13 & 2,331 \\
\hline 14 & 2,371 \\
\hline 15 & 2,409 \\
\hline 16 & 2,443 \\
\hline 17 & 2,475 \\
\hline 18 & 2,504 \\
\hline 19 & 2,532 \\
\hline 20 & 2,557 \\
\hline 21 & 2,580 \\
\hline 22 & 2,603 \\
\hline 23 & 2624 \\
\hline 24 & 2,644 \\
\hline 25 & 2,663 \\
\hline 26 & 2,681 \\
\hline 27 & 2,698 \\
\hline 28 & 2,714 \\
\hline 29 & 2,720 \\
\hline 30 & 2,745 \\
\hline
\end{tabular}

\section{Referências bibliográficas do Anexo A:}

MASSEY JR, F. J. The Kolmogorov-Smirnov test for goodness of fit. Journal of the American statistical Association, v. 46, n. 253, p. 68-78, 1951.

GRUBBS, F. E. Procedures for detecting outlying observations in samples. Technometrics, v. 11, n. 1, p. 1-21, 1969. 